Pacific

Journal of

Mathematics

\title{
CODIMENSION-ONE FOLIATIONS CALIBRATED BY NONDEGENERATE CLOSED 2-FORMS
}

DAVID MARTÍNEZ TORRES 


\title{
CODIMENSION-ONE FOLIATIONS CALIBRATED BY NONDEGENERATE CLOSED 2-FORMS
}

\author{
DAVID MARTÍNEZ TORRES
}

\begin{abstract}
A class of codimension-one foliations has been recently introduced by imposing a natural compatibility condition with a closed maximally nondegenerate 2-form. In this paper we study for such foliations the information captured by a Donaldson-type submanifold. In particular we deduce that their leaf spaces are homeomorphic to leaf spaces of 3-dimensional taut foliations. We also introduce surgery constructions to show that this class of foliations is broad enough. Our techniques come mainly from symplectic geometry.
\end{abstract}

\section{Introduction and statement of main results}

Codimension-one foliations are too large a class of structures to obtain strong structure theorems for them. According to a theorem of Thurston [1976] a closed manifold admits a codimension-one foliation if and only if its Euler characteristic vanishes. In order to draw significant results it is necessary to assume the existence of other structures compatible with the foliation.

From the point of view of symplectic geometry it is natural to consider the following class of codimension-one foliations:

Definition 1 [Ibort and Martínez Torres 2004a]. A codimension-one foliation $\mathscr{F}$ of $M^{2 n+1}$ is said to be 2-calibrated if there exists a closed 2-form $\omega$ such that $\omega_{\mathscr{F}^{n}}{ }^{n}$ is nowhere-vanishing (we also say that $\omega^{n}$ is nowhere-vanishing on $\mathscr{F}$ ).

The 2-calibrated foliation is said to be integral if $[\omega] \in H^{2}(M ; \mathbb{Z})$.

The notation $\omega_{\mathscr{F}}^{n}$ in Definition 1 stands for the restriction of $\omega^{n}$ to the leaves of $\mathscr{F}$. We will be using the subscripts $\mathscr{F}$ and $W$, if $W$ is a submanifold of $M$, to denote the restriction of a form, connection, etc, to the leaves of $\mathscr{F}$ and to $W$,

Partially supported by the Fundação para a Ciência e a Tecnologia (FCT, Portugal), NWO [Symmetries and Deformations in Geometry] (The Netherlands), Pisa University [Galileo Galilei] and Spanish Ministry of Science and Technology [MTM2004-07090-C03].

MSC2010: primary 53C12, 53D17, 57R17, 57R30; secondary 57R65, 53D12.

Keywords: codimension-one foliation, three-dimensional taut foliation, leaf space, symplectic structure, Lagrangian sphere, generalized Dehn twist, Legendrian surgery, Lefschetz pencil. 
respectively. In what follows the manifolds will always be closed and oriented, the codimension-one foliations cooriented and all the structures and maps smooth.

In the next paragraphs we are going to describe how the 2-calibrated condition appears naturally when looking at the problem of constructing submanifolds transverse to a codimension-one foliation.

Recall that a codimension-one foliation $\mathscr{F}$ is said to be taut if every leaf meets a transverse 1-cycle. Tautness in codimension-one can be characterized in several ways using forms, metrics and currents [Sullivan 1976; Rummler 1979; Harvey and Lawson 1982]. The characterization we are interested in says that a rank $p$ codimension-one foliation $\mathscr{F}$ is taut if and only if there exists a closed $p$-form $\xi$ nowhere vanishing on $\mathscr{F}$ (and furthermore according to Proposition 2.7 in [Harvey and Lawson 1982], it is possible to construct a metric $g$ so that $\xi$ is a calibration for $(M, \mathscr{F}))$. Note in particular that a 2 -calibrated foliation $(M, \mathscr{F}, \omega)$ is always taut, since $\xi:=\omega^{n}$ is nowhere-vanishing on $\mathscr{F}$. In dimension three, 2-calibrated foliations are the same as taut foliations.

Let us analyze one direction of the aforementioned characterization: the existence of a closed $p$-form whose restriction to each leaf is a volume form is equivalent to a reduction of the structural pseudogroup of $(M, \mathscr{F})$ to $\operatorname{Vol}\left(\mathbb{R}^{p}, \Xi_{\mathbb{R}^{p}}\right) \times \operatorname{Diff}(\mathbb{R})$, where

$$
\Xi_{\mathbb{R}^{p}}:=d x_{1} \wedge \cdots \wedge d x_{p},
$$

$x_{1}, \ldots, x_{p}$ are coordinates on $\mathbb{R}^{p}$, and $\operatorname{Vol}\left(\mathbb{R}^{p}, \Xi_{\mathbb{R}^{p}}\right)$ and $\operatorname{Diff}(\mathbb{R})$ are the pseudogroups of local diffeomorphisms of $\mathbb{R}^{p}$ and $\mathbb{R}$, respectively, preserving the volume form $\Xi_{\mathbb{R}^{p}}$. Let $U$ be any open subset of a leaf of $\mathscr{F}$. The Poincaré recurrence theorem implies that the flow of any vector field spanning $k e r \xi$ defines a first return map from $U^{\prime} \subset U$ to $U^{\prime \prime} \subset U$. A straightforward consequence is that closed transverse 1-cycles through any given $x \in M$ can be constructed by slightly deflecting integral curves of $\operatorname{ker} \xi$.

The first return map belongs to the pseudogroup $\operatorname{Vol}\left(\mathbb{R}^{p}, \Xi_{\mathbb{R}^{p}}\right)$. If $p=2$, that is, if we have a taut foliation on a 3-manifold, then under certain circumstances we can deduce interesting geometric information about the existence of more closed orbits (Poincaré-Birkhoff theorem). If $p>2$ we have little geometric control on the return map because, assuming for simplicity that $U^{\prime}$ and $U^{\prime \prime}$ are diffeomorphic to a ball, the only invariant is the total volume [Greene and Shiohama 1979, Theorem 1]. Therefore problems such as the existence of transverse submanifolds of dimension bigger than one seem difficult to attack.

It has been known for some time that the right setting to obtain higher dimensional generalizations of Poincaré-Birkhoff theorem is not volume geometry but symplectic geometry [Hofer and Zehnder 1994, Chapter 6; McDuff and Salamon 1998, Chapter IV]. It can be checked (see Section 2) that the existence of a closed 2-form $\omega$ which makes the leaves of $(M, \mathscr{F})$ symplectic manifolds amounts to a reduction 
of the structural pseudogroup of $(M, \mathscr{F})$ to $\operatorname{Symp}\left(\mathbb{R}^{2 n}, \Omega_{\mathbb{R}^{2 n}}\right) \times \operatorname{Diff}(\mathbb{R})$, where $\operatorname{Symp}\left(\mathbb{R}^{2 n}, \Omega_{\mathbb{R}^{2 n}}\right)$ is the pseudogroup of local diffeomorphisms of $\mathbb{R}^{2 n}$ preserving the standard symplectic form

$$
\Omega_{\mathbb{R}^{2 n}}:=\sum_{i=1}^{n} d x_{i} \wedge d y_{i} .
$$

Thus, return maps associated to the flow of vector fields generating $\operatorname{ker} \omega$ belong to $\operatorname{Symp}\left(\mathbb{R}^{2 n}, \Omega_{\mathbb{R}^{2 n}}\right)$. Symplectomorphisms are much more rigid than transformations preserving the volume form $\Omega_{\mathbb{R}^{2 n}}^{n}=n ! \Xi_{\mathbb{R}^{2 n}}$. They preserve the symplectic invariants of subsets of $\mathbb{R}^{2 n}$, so for example these cannot be squeezed along symplectic 2planes [Hofer and Zehnder 1994, Chapters 2 and 3; McDuff and Salamon 1998, Section 12]. Naively, one might try to construct transverse 3-manifolds by choosing tiny 2-dimensional symplectic pieces $\Sigma$ inside a leaf, whose image by the first return map is a small 2-dimensional symplectic manifold that can be isotoped to $\Sigma$ through symplectic surfaces. The isotopy would be used to connect both symplectic surfaces in nearby leaves, and thus get a piece of transverse 3-dimensional taut foliation. Of course this idea seems difficult to be carried out because different pieces should be combined to construct a closed 3-manifold. However, it provides some insight on why 2-calibrated foliations are expected to have embedded 3-dimensional taut foliations.

In [Ibort and Martínez Torres 2004a, Corollary 1.2], it was proved that for any 2-calibrated foliation $(M, \mathscr{F}, \omega)$ there exists an embedding of a 3-dimensional submanifold $W^{3} \hookrightarrow M$, such that $W^{3}$ is transverse to $\mathscr{F}$ and $\omega_{W}$ is nowhere vanishing on $\mathscr{F}_{W}$; the 3 -dimensional submanifold $W^{3}$, which inherits a taut foliation, is a Donaldson-type submanifold [Donaldson 1996; Auroux 1997]. Its existence is an elementary consequence of the extension to 2-calibrated foliations of the approximately holomorphic techniques for symplectic manifolds introduced by Donaldson [1996].

1.1. Statement of results. Take $(M, \mathscr{F}, \omega)$ to be a 2 -calibrated foliation, and let $W \hookrightarrow(M, \mathscr{F})$ be a 3-dimensional Donaldson-type submanifold. In this paper we are mainly concerned with finding out which properties of $(M, \mathscr{F})$ are captured by $W$.

If $F$ is a compact leaf of $(M, \mathscr{F}, \omega)$, an appropriate version of the Lefschetz hyperplane theorem [Donaldson 1996, Proposition 39] asserts that $W \cap F$ is connected. A codimension-one foliation $(M, \mathscr{F})$ has noncompact leaves unless it is a fibration over the circle (a mapping torus). If $F$ is a noncompact leaf then describing global properties of $W \cap F$ seems very difficult. Our main result is a rather surprising and counterintuitive global property of such intersections for appropriate Donaldson-type submanifolds. 
Theorem 2. Let $(M, \mathscr{F}, \omega)$ be a 2-calibrated foliation. Then there exist Donaldsontype submanifolds $W^{3} \hookrightarrow(M, \mathscr{F})$, such that for every leaf $F$ of $\mathscr{F}$ the intersection $W \cap F$ is connected.

Remark 3. Any integral 2-calibrated foliation $(M, \mathscr{F}, \omega)$ admits embeddings in complex projective spaces $\mathbb{C P}^{N}$ of large dimension, with the property that the ambient Fubini-Study symplectic form restricts to a multiple of $\omega$ [Ibort and Martínez Torres 2004a, Corollary 1.3]. The 3-dimensional transverse submanifolds in Theorem 2 can be arranged to appear as intersections of $M \subset \mathbb{C P}^{N}$ with appropriate projective subspaces. Theorem 2 should be understood as a leafwise Lefschetz hyperplane-type result for $\pi_{0}$.

An important consequence of Theorem 2 is the following result:

Theorem 4. Let $(M, \mathscr{F}, \omega)$ be a 2-calibrated foliation. There exists a 3-dimensional embedded taut foliation such that the inclusion $\left(W^{3}, \mathscr{F}_{W}\right) \hookrightarrow(M, \mathscr{F})$ descends to a homeomorphism of leaf spaces $W / \mathscr{F}_{W} \rightarrow M / \mathscr{F}$.

Thus, leaf spaces of 2-calibrated foliations are no more complicated than those of 3-dimensional taut foliations.

A second goal of this paper is showing that 2-calibrated foliations are a broad enough class of foliations. In this respect there are three basic families of 2-calibrated foliations: products, cosymplectic foliations and symplectic bundle foliations.

In a product we cross a 2-calibrated foliation - typically a 3-dimensional taut foliation - with a (nontrivial) symplectic manifold, and put the product foliation and the obvious closed 2-form.

A cosymplectic foliation is a triple $(M, \alpha, \omega)$, where $\alpha$ is a nowhere vanishing closed 1-form and $(M, \operatorname{ker} \alpha, \omega)$ is a 2-calibrated foliation.

A bundle foliation with fiber $S^{1}$ is by definition an $S^{1}$-fiber bundle $\pi: M \rightarrow X$ endowed with a codimension-one foliation $\mathscr{F}$ transverse to the fibers. If the base space admits a symplectic form $\sigma$, then $\left(M, \mathscr{F}, \pi^{*} \sigma\right)$ is a 2-calibrated foliation which we refer to as a symplectic bundle foliation.

The second topic of this paper concerns the introduction of two surgery constructions for 2-calibrated foliations: normal connected sum and generalized Dehn surgery or Lagrangian surgery. Using surgery we have obtained the following result:

Proposition 5. There exist 2-calibrated foliations (of dimension bigger than three) which are neither products, nor cosymplectic foliations, nor symplectic bundle foliations.

The paper is organized as follows. In Section 2 we introduce definitions and basic facts on 2-calibrated foliations, and address their relation to regular Poisson structures. 
Section 3 describes how to adapt the normal connected sum for symplectic and Poisson manifolds to integral 2-calibrated foliations; this is the surgery used to prove Proposition 5.

In Section 4 we present a surgery based on generalized Dehn twists. Generalized Dehn surgery is the natural extension to 2-calibrated foliations of positive Dehn surgery along a curve in a leaf of a 3-dimensional taut foliation $\left(M^{3}, \mathscr{F}\right)$.

It is a classical result of Lickorish [1965] that positive Dehn surgery along a curve $\gamma$ has an alternative description: $\gamma$ carries a canonical framing and therefore it determines an elementary cobordism from $M^{3}$ to $M^{\prime}$, which amounts to attaching a 2-handle to the trivial cobordism $M \times[0,1]$. The "new" boundary component $M^{\prime}$ is endowed with a canonical foliation which coincides with positive Dehn surgery on $(M, \mathscr{F})$ along $\gamma$.

If $\left(M^{2 n+1}, \mathscr{F}, \omega\right)$ is a 2 -calibrated foliation, a parametrized Lagrangian $n$-sphere inside a leaf of $\mathscr{F}$ canonically determines the attaching of a $(n+1)$-handle. We show that the corresponding elementary $(2 n+2)$-dimensional cobordism admits a symplectic structure, which induces a 2-calibrated foliation on the new boundary component of the cobordism. We call this construction Lagrangian surgery. In Theorem 26 we extend Lickorish's result by proving that generalized Dehn surgery and Lagrangian surgery produce equivalent 2 -calibrated foliations. The importance of this result stems from the fact that the aforementioned symplectic elementary cobordisms do appear in a natural way associated to Lefschetz pencil structures. As a byproduct we get an application to contact geometry that we have included in an appendix: it is a proof of a result announced by Giroux and Mohsen [2003], relating generalized Dehn surgery along a parametrized Lagrangian sphere $L$ in an open book decomposition compatible with a contact structure, and Legendrian surgery along $L$. Results in this section require a fine analysis of the symplectic monodromy about the singular fiber of the complex quadratic form.

In Section 5 we prove Theorems 2 and 4. The main tools are Lefschetz pencil structures for $(M, \mathscr{F}, \omega)$, which are appropriate analogs of leafwise complex Morse functions and whose existence is an application of approximately holomorphic geometry for 2-calibrated foliations. A regular fiber of a Lefschetz pencil structure is a Donaldson-type submanifold. A Lefschetz pencil structure admits a leafwise symplectic connection. Its associated leafwise symplectic parallel transport is the key ingredient to prove our main theorem relating the leaf space of any regular fiber of the pencil to the leaf space of $(M, \mathscr{F}, \omega)$. Symplectic parallel transport also allows us to compare the 2-calibrated foliations induced on different regular fibers. Namely, in Theorem 37 we show that any two regular fibers of a Lefschetz pencil structure for $(M, \mathscr{F}, \omega)$ are related by a sequence of symplectic handle attachings along Lagrangian spheres. By the symplectic analog of Lickorish's result proved in Section 4, we conclude that any two regular fibers of a Lefschetz pencil structure 
are related by a sequence of generalized Dehn surgeries. We finish the section by discussing some open problems.

\section{Definitions and basic results}

In this section we introduce some basic definitions, results and examples. We also address the relation of 2-calibrated foliations to Poisson structures.

Definition 6. Let $(M, \mathscr{F}, \omega)$ be a 2-calibrated foliation and let $l: N \hookrightarrow M$ be a submanifold. We say that $N$ is a 2-calibrated submanifold if $\left(N, l^{*} \mathscr{F}, l^{*} \omega\right)$ is a 2-calibrated foliation.

The definition of a 2-calibrated foliation can be given locally.

Definition 7. A 2-calibration for $(M, \mathscr{F})$ is a reduction of its structural pseudogroup to $\operatorname{Symp}\left(\mathbb{R}^{2 n}, \Omega_{\mathbb{R}^{2 n}}\right) \times \operatorname{Diff}(\mathbb{R})$.

Definitions 1 and 7 are equivalent. A standard Darboux-type result (see for example [McDuff and Salamon 1998, Chapter 3] for basic material on symplectic geometry) implies that about any point in $M$, there exists a foliated chart with coordinates $x_{1}, y_{1}, \ldots, x_{n}, y_{n}, t$ (the image of $\mathscr{F}$ in $\mathbb{R}^{2 n+1}$ is the foliation by affine hyperplanes with constant coordinate $t$ ), such that $\omega$ is the pullback of

$$
\omega_{\mathbb{R}^{2 n+1}}:=\sum_{i=1}^{n} d x_{i} \wedge d y_{i} .
$$

It is clear that on a given manifold, 2-calibrated foliations are an open subset of the set of codimension-one foliations in the $C^{0}$-topology. More precisely, in the product space of codimension-one foliations and closed 2-forms, pairs corresponding to 2-calibrated foliations are an open set in the $C^{0}$-topology.

The first examples of 2-calibrated manifolds are 3-dimensional taut foliations. In this paper we are concerned with higher dimensional 2-calibrated foliations. An elementary family is obtained by applying the product construction to 3-dimensional taut foliations and nontrivial symplectic manifolds.

Another important family of 2-calibrated foliations are cosymplectic foliations. Recall that they are given by a triple $\left(M^{2 n+1}, \alpha, \omega\right), \alpha$ a closed 1-form and $\omega$ a closed 2-form such that $\alpha \wedge \omega^{n}$ is a volume form. An example of a cosymplectic foliation is a 2-calibrated foliation whose leaves are the fibers of a fibration over the circle; the closed 1-form defining the foliation is the pullback of any volume form on the circle. Each fiber is a closed symplectic manifold and the first return map associated to the kernel of the calibrating 2-form is a symplectomorphism. We refer to such cosymplectic foliations as symplectic mapping tori. In fact, symplectic mapping tori are characterized as cosymplectic foliations whose defining 1-form has rank one period lattice. This characterization implies that symplectic mapping 
tori are $C^{0}$-dense in cosymplectic foliations. The reason is that the defining 1 -form can be approximated by closed 1 -forms with rational periods.

Cosymplectic foliations appear naturally in symplectic geometry as follows: recall that a vector field $Y$ on a symplectic manifold $(Z, \Omega)$ is called symplectic if $L_{Y} \Omega=0$. If $Y$ is a symplectic vector field transverse to $\partial Z$, then its symplectic annihilator

$$
\operatorname{Ann}(Y)^{\Omega}=\{v \in T Z \mid \Omega(Y, v)=0\}
$$

is an integrable codimension-one distribution. Since it contains the vector field $Y$, it induces a codimension-one foliation $\mathscr{F}$ on $\partial M$. Let $\alpha:=i_{Y} \Omega$. It can be checked that $\left(\partial M, \alpha_{\partial M}, \Omega_{\partial M}\right)$ is a cosymplectic foliation.

The previous construction leads to an analogy between cosymplectic foliations and contact structures. The reason is that on a symplectic manifold $(Z, \Omega)$ endowed with a vector field $Y$ transverse to the boundary and satisfying $L_{Y} \Omega=\Omega$, the restriction of $i_{Y} \Omega$ to $\partial M$ is a contact form. Following this analogy, we define the Reeb vector field $R$ of a cosymplectic foliation $(M, \alpha, \omega)$ to be the vector field characterized by the equations $i_{R} \omega=0, i_{R} \alpha=1$. The foliation is invariant under the flow of the Reeb vector field. In fact, a cosymplectic foliation can be defined as a 2-calibrated foliation endowed with a vector field $R$ spanning the kernel of $\omega$ and whose flow preserves the foliation; we say that $R$ is a Reeb vector field.

A third family of 2-calibrated foliations are symplectic bundle foliations, ${ }^{1}$ which are defined as bundle foliations with fiber $S^{1}$ over symplectic manifolds. There is a very rough way of associating symplectic bundle foliations to any bundle foliation $\pi: M \rightarrow X$ with fiber $S^{1}$. The latter is characterized by a conjugacy class of representations of $\pi_{1}(X, x)$ in $\operatorname{Diff}\left(S^{1}\right)$. A result of Gompf [1995, Theorem 0.1 ] asserts that there exist closed symplectic manifolds (of dimension 4) whose fundamental group isomorphic to $\pi_{1}(X, x)$.

Example 8. Let $x_{1}, y_{1}, x_{2}, y_{2}, t$ be coordinates on $\mathbb{R}^{5}$ and consider the canonical 2-form $\omega_{\mathbb{R}^{5}}$. It descends to a closed 2-form $\omega_{\mathbb{T}^{5}}$, where $\mathbb{T}^{5}=\mathbb{R}^{5} / \mathbb{Z}^{5}$. Let $\mathscr{F}$ be any of the foliations on $\mathbb{T}^{5}$ induced by a constant 1 -form $\alpha$ on $\mathbb{R}^{5}$ whose kernel is transverse to $\partial / \partial t$. Then $\left(\mathbb{T}^{5}, \alpha, \omega_{\pi^{5}}\right)$ is a 2-calibrated foliation. Its leaves are all diffeomorphic to $\mathbb{R}^{i} \times \mathbb{T}^{4-i}$, where $i \in\{0, \ldots, 4\}$ depends on the slopes of the kernel of the 1-form.

By construction $\left(\mathbb{T}^{5}, \alpha, \omega_{\mathbb{T}^{5}}\right)$ is both a cosymplectic foliation and a symplectic bundle foliation. It is a product (respectively a mapping torus) if and only if the leaves are diffeomorphic to $\mathbb{R}^{i} \times \mathbb{T}^{4-i}, i \leq 2$ (respectively $\mathbb{T}^{4}$ ).

Deciding which manifolds admit a 2-calibrated foliation can be divided in several subproblems which in general are very hard. A 2-calibrated foliation $(M, \mathscr{F}, \omega)$

\footnotetext{
${ }^{1}$ This family of 2-calibrated foliations was pointed out to the author by the referee.
} 
is the superposition of several compatible structures. Firstly, there is the foliation. Secondly, the 2-form restricts to a closed nondegenerate foliated 2-form $\omega_{\mathscr{F}}$. The pair $\left(\mathscr{F}, \omega_{\mathscr{F}}\right)$ defines a (regular) Poisson structure on $M$ and as such it is also defined by an appropriate bivector field $\Pi$. And thirdly, the foliated symplectic form $\omega_{\mathscr{F}}$ admits a lift to a global closed 2-form $\omega$.

Determining which codimension-one foliations are the symplectic foliations of a Poisson structure is very complicated; there exist partial results which use h-principles and only apply to open manifolds [Bertelson 2001; Bertelson 2002; Fernandes and Frejlich 2012]. The existence of a closed lift of a foliated 2-form $\omega_{\mathscr{F}}$ is controlled by three obstructions associated to the spectral sequence which relates basic cohomology, leafwise cohomology and the cohomology of the total space [El Kacimi-Alaoui 1983] (see [Alcalde-Cuesta and Hector 1993] for a treatment in the setting of Poisson geometry); if the foliation is defined by a closed 1-form, then the obstruction to the existence of a closed lift admits a simpler description [Guillemin et al. 2011, Section 2.2].

We would like to regard a 2-calibrated foliation as a codimension-one regular Poisson manifold with a lift of $\omega_{\mathscr{F}}$ to a closed 2-form $\omega$. We are not fully interested in the 2-form $\omega$, as the following definition reflects.

Definition 9. Let $\left(M_{j}, \mathscr{F}_{j}, \omega_{j}\right), j=1,2$, be 2-calibrated foliations. They are said to be equivalent if there exists a diffeomorphism $\phi: M_{1} \rightarrow M_{2}$ such that

- $\phi$ is a Poisson morphism or equivalence (it preserves the foliations together with the leafwise 2-forms),

- $\left[\phi^{*} \omega_{2}\right]=\left[\omega_{1}\right] \in H^{2}\left(M_{1} ; \mathbb{R}\right)$ and $\phi$ preserves the coorientations.

For symplectic mapping tori, an equivalence is just a Poisson diffeomorphism preserving coorientations. Alternatively, equivalent symplectic mapping tori are those with the same symplectic leaf and isotopic first return maps (the isotopy being through symplectomorphisms).

As we shall see in the following sections, the notion of equivalence is the right one to remove the dependence on choices in our surgeries.

\section{Normal connected sum}

In the previous section we saw that deciding whether a manifold supports a 2calibrated foliation is very complicated. It is thus natural to look for procedures to build new 2-calibrated foliations out of given ones. In this section we introduce the normal connected sum of integral 2-calibrated foliations, and we use it to give examples of 2-calibrated foliations which do not belong to either of the three elementary families, hence proving Proposition 5. 
Symplectic normal connected sum is a surgery construction in which two symplectic manifolds are glued along two copies of the same codimension-two symplectic submanifold, which enters in the manifolds with opposite normal bundles [Gompf 1995, Theorem 1.3]. A parametric version of this surgery gives rise to an analogous construction for regular Poisson manifolds [Ibort and Martínez Torres 2003, Theorem 1]. We propose the following extension to integral 2-calibrated foliations. Theorem 10. Let $\left(M_{j}^{2 n+1}, \mathscr{F}_{j}, \omega_{j}\right), j=1,2$, be integral 2-calibrated foliations. Let $\left(N^{2 n-1}, \mathscr{F}_{N}, \omega_{N}\right)$ be a 2 -calibrated foliation which is a symplectic mapping torus. Assume that we have maps $l_{j}: N \hookrightarrow M_{j}, j=1,2$, embedding $N$ as a 2-calibrated submanifold of $M_{j}$ (Definition 6 ), such that the following properties hold:

(i) The 2-calibrated foliations induced by the embeddings are equivalent to the given one $\left(N, \mathscr{F}_{N}, \omega_{N}\right)$ (Definition 9).

(ii) The normal bundles of $l_{j}(N) \subset M_{j}, j=1,2$, are trivial.

(iii) The fiber of $N \rightarrow S^{1}$ is simply connected.

Then there exist gluing maps $\psi$ such that the Poisson structure П on $M_{1} \#_{\psi} M_{2}$ characterized by matching on $M_{j} \backslash l_{j}(N)$ the Poisson structures $\Pi_{j}$ associated to $\left(M_{j}, \mathscr{F}_{j}, \omega_{j}\right), j=1,2$, admits a lift to a 2-calibrated structure.

Proof. By assumptions (i) and (ii) Poisson surgery produces a Poisson structure $\Pi$ on $M_{1} \#_{\psi} M_{2}$ [Ibort and Martínez Torres 2003]. Very briefly, there is a gluing map $\psi$ identifying $A_{1} \rightarrow A_{2}$ annular neighborhoods of $l_{1}(N)$ and $l_{2}(N)$ (by this we mean tubular neighborhoods from which we remove $\left.l_{j}(N), j=1,2\right)$ defined as follows: by assumption (ii) the normal bundles are trivial and by Darboux-Weinstein theorem with parameters the (smooth) leaf space of $N$ [McDuff and Salamon 1998, Chapter 3 ], there exist trivializations in which $\Pi_{j}, j=1,2$, split. One factor is the leafwise symplectic form on $l_{j}(N)$ and the other one is the standard symplectic form $d x \wedge d y$ on the normal disk with coordinates $x, y$. On each normal disk $\psi$ is the unique rotationally independent symplectomorphism of the punctured disk of radius $\delta>0$ which reverses the orientation of the radii.

Let $\left(\mathscr{F}, \omega_{\mathscr{F}}\right)$ denote the foliation and leafwise symplectic form associated to $\Pi$. If there is a lift of $\omega_{\mathscr{F}}$ to an integral closed 2-form $\omega$, then there must be a Hermitian line bundle $L$ and a compatible connection $\nabla$ such that $-2 \pi i \omega=F_{\nabla}$, where $F_{\nabla}$ is the curvature of the connection.

Because the $w_{j}, j=1,2$, represent integral cohomology classes, there exist Hermitian line bundles $\left(L_{j}, \nabla_{j}\right) \rightarrow M_{j}$ with compatible connections such that

$$
-2 \pi i \omega_{j}=F_{\nabla_{j}} .
$$

We look for a lift of $\psi$ to a bundle isomorphism $\Psi: L_{1 \mid A_{1}} \rightarrow L_{2 \mid A_{2}}$ to define a (Hermitian) line bundle $L:=L_{1} \#_{\Psi} L_{2} \rightarrow M_{1} \#_{\psi} M_{2}$. Let $c_{j}, j=1$, 2, denote the 
Chern classes of $L_{j \mid A_{j}}$, which are integral lifts of the restrictions of $w_{j}$ to $A_{j}$. An isomorphism lifting $\psi$ exists if and only if

$$
\psi^{*} c_{2}=c_{1} \in H^{2}\left(A_{1} ; \mathbb{Z}\right) .
$$

Because the fiber of $N \rightarrow S^{1}$ is simply connected, the Wang sequence for the mapping torus $A_{1} \rightarrow S^{1}$ implies that $H^{2}\left(A_{1} ; \mathbb{Z}\right)$ is torsion free. Therefore (2) is equivalent to

$$
\left[\psi^{*} w_{2 \mid A_{2}}\right]=\left[w_{1 \mid A_{1}}\right] \in H^{2}\left(A_{1} ; \mathbb{R}\right) .
$$

Because the $w_{j}, j=1,2$, extend to $A_{j} \cup l_{j}$ and the cohomology of the tubular neighborhoods is concentrated in $l_{j}(N)$, (3) is equivalent to

$$
\left[l_{2}^{*} w_{2}\right]=\left[l_{1}^{*} w_{1}\right] \in H^{2}(N ; \mathbb{R}),
$$

which holds true because by assumption (i) the 2-calibrations induced by $l_{1}$ and $l_{2}$ on $N$ are equivalent.

Therefore we obtain $L \rightarrow M_{1} \#_{\psi} M_{2}$ a Hermitian line bundle with two not everywhere defined compatible connections $\nabla_{1}, \nabla_{2}$, overlapping on $A_{1} \subset M_{1} \#_{\psi} M_{2}$. Note that by (1) the leafwise curvatures match on $A_{1}$. We are going to use the assumptions to modify $\nabla_{1}$ and $\nabla_{2}$ (the latter away from $l_{2}(N)$ ), so that we obtain the leafwise equality of connections on $A_{1}$. Then a convex combination of both connections associated to a partition of the unity subordinated to $M_{j} \backslash l_{j}(N), j=1,2$, is a connection on $M_{1} \#_{\psi} M_{2}$ whose leafwise curvature is $-2 \pi i \omega \mathscr{F}$.

The difference

$$
l_{1}^{*} \nabla_{1}-l_{2}^{*} \nabla_{2}
$$

is a leafwise closed 1-form on $N$ (recall that $N$ is a mapping torus and therefore all leaves are compact). By assumption (iii) it is leafwise exact and therefore we can modify say $\nabla_{2}$, by adding a smooth leafwise primitive function so the 1-form in (4) is leafwise vanishing.

Triviality of the normal bundles implies the existence of normal forms for the leafwise connections on tubular neighborhoods of $l_{j}(N), j=1,2$, which only depend on the restrictions of the leafwise connections to $l_{j}(N)$; the normal forms amount to fixing a primitive 1 -form for $d x \wedge d y$. The connections can be assumed to coincide with the normal forms. Finally the difference $\nabla_{1}-\psi^{*} \nabla_{2}$ is not still leafwise vanishing; on each normal annulus it is the differential of an (explicit) function, and what we do is modify $\nabla_{2}$ accordingly on $M_{2} \backslash l_{2}(N)$.

As for dependence of the construction on choices, remark that the choice of isotopy classes of trivializations of the normal bundles (the framings), may affect the diffeomorphism class of $M_{1} \#_{\psi} M_{2}$. For fixed isotopy classes of trivializations of the normal bundles, the underlying Poisson structure is unique up to Poisson 
diffeomorphism. The reason is that the leafwise symplectic form is unique up to isotopy supported near $N$. This follows from an elementary argument which is going to be used several times: because the leaves of $N$ have no first cohomology group, the local path of symplectomorphisms provided by Moser's argument is Hamiltonian [McDuff and Salamon 1998, Chapter 3]. The choice of primitive Hamiltonian function can be done coherently for all leaves of $N$. By extending the corresponding function to a global one supported near $N$, we construct a path of transformations connecting both Poisson structures. Also, if we fix an isotopy class of lifts $\Psi$, the 2-calibrated structure provided by the normal connected sum is unique up to equivalence. This is because the cohomology class of the calibrating 2 -form is the image in real cohomology of the first Chern class of the bundle $L$, which is fixed by the choice of isotopy class of lifts.

Remark 11. The hypotheses needed to define normal connected sum of regular Poisson manifolds are much weaker than the requirements in Theorem 10. In particular the normal bundles $l_{j}(N), j=1,2$, are not required to be trivial, just opposite. Triviality of the normal bundles is necessary if we want to produce an integral 2-calibrated foliation extending the given Poisson structures $\Pi_{j}$ on $M_{j} \backslash l_{j}(N), j=1,2$. The reason is that already in the symplectic setting, having nontrivial normal bundle gives rise to choices in the construction which result is symplectic forms with different volume; this is a well-known issue that appears when blowing up symplectic submanifolds [McDuff and Salamon 1998, Chapter 7].

Perhaps the assumptions in Theorem 10 can be weakened if we just require the existence of a 2-calibration on the normal connected sum.

The normal connected sum can be applied to construct integral 2-calibrated foliations that use as building blocks 2-calibrated foliations which are products and symplectic mapping tori, but which are neither products, nor cosymplectic foliations nor symplectic bundle foliations.

Proof of Proposition 5. Let $\left(P^{4}, \Omega\right)$ be an integral symplectic 4-manifold which contains a symplectic sphere $S^{2}$ with trivial normal bundle; let $A \in \mathbb{Z}$ be the induced area form on the sphere. Let $\varphi \in \operatorname{Symp}(P, \Omega)$ such that $\varphi_{\mid S^{2}}=\mathrm{Id}$; for example $\varphi$ can be the identity. We define $\left(M_{1}, \mathscr{F}_{1}, \omega_{1}\right)$ to be the symplectic mapping torus associated to $\varphi$.

Let $\left(M_{2}, \mathscr{F}_{2}, \omega_{2}\right)$ be the product 2-calibrated foliation with factors any taut foliation $\left(Y^{3}, \mathscr{F}^{3}, \sigma\right)$ and the sphere $\left(S^{2}, A\right)$; via a small perturbation and a rescaling of $\sigma$, we may take $\omega_{2}$ to be integral. Let $C$ be a fixed transverse cycle for $\left(Y^{3}, \mathscr{F}^{3}, \sigma\right)$ and $\theta: S^{1} \rightarrow C$ any fixed positive parametrization with respect to the coorientation.

Let $N^{3}$ be the result of applying the mapping torus construction to

$$
\operatorname{Id} \in \operatorname{Symp}\left(S^{2}, A\right), \quad \text { where } N \cong S^{1} \times S^{2} .
$$


Since $\varphi_{\mid S^{2}}=\mathrm{Id}$, there is an obvious embedding $l_{1}: N \hookrightarrow M_{1}$. The embedding $l_{2}$ is the product map $\theta \times \mathrm{Id}: N \hookrightarrow M_{2}$.

By construction the embeddings fulfill the hypothesis of Theorem 10, so we obtain a 2-calibrated foliation $\left(M_{1} \#_{\psi} M_{2}, \mathscr{F}, \omega\right)$.

We impose the following additional constraints on the summands to make sure that $\left(M_{1} \#_{\psi} M_{2}, \mathscr{F}_{F}, \omega\right)$ does not belong to the three basic families:

- $\left(Y^{3}, \mathscr{F}^{3}\right)$ contains compact and noncompact leaves.

- There is a compact leaf $\Sigma$ of $\left(Y^{3}, \mathscr{F}^{3}\right)$ which intersects $C$ in exactly one point, and $\left(P^{4}, \Omega\right)$ is an odd Hirzebruch surface [McDuff and Salamon 1998, Chapter 4].

- The genus of $\Sigma$ is greater than one, and $\pi_{1}(Y)$ is not isomorphic to $\pi_{1}\left(S^{1} \times \Sigma\right)$.

Because $l_{2}(N)$ intersects each leaf of $\left(M_{2}, \mathscr{F}_{2}\right)$ in a unique connected component, there is a one to one correspondence between leaves of $\left(Y^{3}, \mathscr{F}^{3}\right)$ and leaves of $\left(M_{1} \#_{\psi} M_{2}, \mathscr{F}\right)$. This correspondence sends a leaf $F$ of $\left(Y^{3}, \mathscr{F}^{3}\right)$ to the leaf which contains $\left(F \times S^{2}\right) \backslash\left(l_{2}(N) \cap\left(F \times S^{2}\right)\right)$. Because the leaves of $\left(M_{2}, \mathscr{F}_{2}\right)$ are compact, the correspondence sends compact leaves to compact leaves and noncompact leaves to noncompact leaves. Since $\left(Y^{3}, \mathscr{F}^{3}\right)$ contains compact and noncompact leaves, so does $\left(M_{1} \#_{\psi} M_{2}, \mathscr{F}_{F}, \omega\right)$, and hence it has nontrivial holonomy. Consequently, $\left(M_{1} \#_{\psi} M_{2}, \mathscr{F}, \omega\right)$ cannot be a cosymplectic foliation.

Let $\Sigma$ be a compact leaf of $\mathscr{F}^{3}$ which intersects $C$ in one point. The correspondence between leaves described in the previous paragraph sends $\Sigma$ to a compact leaf $F_{\Sigma}$, which is the symplectic normal connected sum of the odd Hirzebruch surface and $\left(\Sigma \times S^{2}, p_{1}^{*} \omega_{\mid \Sigma}+p_{2}^{*} A\right)$ along a symplectic sphere with trivial normal bundle. At the differentiable level $F_{\Sigma}$ is the normal connected sum of the trivial $S^{2}$-fibration over $\Sigma$ and the twisted $S^{2}$-fibration over $S^{2}$, and hence it is the twisted $S^{2}$-fibration over $\Sigma$ (the fibers of our fibrations have a coherent orientation, since they are symplectic). If $F_{\Sigma}$ is diffeomorphic to a product of surfaces then we can only have $F_{\Sigma} \cong S^{2} \times \Sigma$; otherwise we could not have isomorphic fundamental groups. But then $F_{\Sigma}$ would admit two different $S^{2}$-fibration structures, and this is in contradiction with [Melvin 1984]. Therefore $\left(M_{1} \#_{\psi} M_{2}, \mathscr{F}_{F}, \omega\right)$ cannot be a product.

If the normal connected sum is a symplectic bundle foliation $\pi: M_{1} \#_{\psi} M_{2} \rightarrow X$, then $F_{\Sigma}$ is a covering space of $X$. Because the fundamental group of $F_{\Sigma}$ is the fundamental group of $\Sigma$, our assumption on the genus of $\Sigma$ implies that the covering must be trivial. Therefore $\pi$ sends $F_{\Sigma}$ diffeomorphically onto $X$. This also implies that the principal $S^{1}$-bundle has a section, so $M_{1} \#_{\psi} M_{2}$ is the trivial bundle $S^{1} \times F_{\Sigma}$. Hence $\pi_{1}\left(M_{1} \#_{\psi} M_{2}\right)$ is diffeomorphic to $\pi_{1}\left(S^{1} \times \Sigma\right)$. But applying Seifert-Van Kampen theorem to the open subsets $M_{1} \backslash l_{1}(N), M_{2} \backslash l_{2}(N)$ gives that $\pi_{1}\left(M_{1} \#_{\psi} M_{2}\right)$ is diffeomorphic to $\pi_{1}(Y)$, and this contradicts the assumption on $\pi_{1}(Y)$. 


\section{Generalized Dehn surgery}

In this section we introduce our second surgery, generalized Dehn surgery. We give a first definition which is the most natural one from the viewpoint of foliation theory. We present a second approach via handle-attaching along Lagrangian spheres; this is a very natural definition taking into account the description of Legendrian surgeries in contact geometry [Weinstein 1991, Elementary Cobordisms Section]. We prove the equivalence of both constructions in Theorem 26.

Generalized Dehn surgery is done, unlike normal connected sum, along a submanifold inside one of the leaves. Let $(M, \mathscr{F}, \omega)$ be a 2-calibrated foliation. We orient $M$ so that a positive transverse vector followed by a positive basis of the leaf with respect to the volume form $\omega_{\mathscr{F}}^{n}$ gives a positive basis.

Let $T:=T^{*} S^{n}$ and $d \alpha_{\text {can }}$ its canonical symplectic structure. Let $\tau: T \rightarrow T$ be a generalized Dehn twist. Recall that these are certain compactly supported symplectomorphisms of $\left(T, d \alpha_{\text {can }}\right)$ which induce the antipodal map on the zero section. Let $T(\lambda)$ be the subset of cotangent vectors of length at most $\lambda$ with respect to the round metric. Generalized Dehn twists can be chosen to be supported in the interior of $T(\lambda)$ for any fixed $\lambda$, and any two with such property are isotopic in $\operatorname{Symp}^{\text {comp }}\left(T(\lambda), d \alpha_{\text {can }}\right)$, the group of compactly supported symplectomorphisms [Seidel 2003, Lemma 1.10 in Section 1.2]. They are symplectic generalizations of Dehn twists on $T^{*} S^{1}$.

A parametrized Lagrangian sphere $L \subset(M, \mathscr{F}, \omega)$ is a submanifold of a leaf $F_{L}$ such that $\omega_{L} \equiv 0$, together with a parametrization $l: S^{n} \rightarrow L$. By a theorem of Weinstein [McDuff and Salamon 1998, Chapter 3], there exists $U$ a compact neighborhood of $L$ inside $F_{L}$ and $\lambda>0$, such that $l^{-1}: L \rightarrow S^{n}$ extends to a symplectomorphism $\varphi:\left(U, \omega_{\mathscr{F}}\right) \rightarrow\left(T(\lambda), d \alpha_{\text {can }}\right)$. Let us assume that if $n=1$ the loop $L$ has trivial holonomy; if $n>1$ the absence of holonomy is a consequence of Reeb's theorem. In a neighborhood of $L$ the foliation is a product. We let $R$ be a local positive Reeb vector field and we let $\Phi_{t}^{R}$ denote its time $t$ flow, which by definition preserves $\mathscr{F}$. Let $\epsilon>0$ small enough so that

$$
\begin{aligned}
\Phi^{R}:[-\epsilon, \epsilon] \times U & \rightarrow M, \\
(t, x) & \mapsto \Phi_{t}^{R}(x)
\end{aligned}
$$

is an embedding. We introduce the following notation:

$$
\begin{aligned}
U(\epsilon) & :=\Phi^{R}([-\epsilon, \epsilon] \times U), & U_{t} & :=\Phi_{t}^{R}(U), \\
U^{+}(\epsilon) & :=\Phi^{R}([0, \epsilon] \times U), & U^{-}(\epsilon) & :=\Phi^{R}([-\epsilon, 0] \times U) .
\end{aligned}
$$

The result of cutting $U(\epsilon)$ along $U$ is the manifold $U^{-}(\epsilon) \amalg U^{+}(\epsilon)$ whose boundary contains $U^{-}=U \times\{0\} \subset U^{-}(\epsilon)$ and $U^{+}=U \times\{0\} \subset U^{+}(\epsilon)$. 
Definition 12. Let $L \subset(M, \mathscr{F}, \omega)$ be a parametrized Lagrangian sphere. If $n=1$, assume that $L$ is a loop with trivial holonomy. Generalized Dehn surgery along $L$ is defined by cutting $M$ along $U$ as above and then gluing back via the composition

$$
\chi:\left(U^{-}, \omega \mathscr{F}_{F} \stackrel{\varphi}{\rightarrow}\left(T(\lambda), d \alpha_{\mathrm{can}}\right) \stackrel{\tau}{\rightarrow}\left(T(\lambda), d \alpha_{\mathrm{can}}\right) \stackrel{\varphi^{-1}}{\rightarrow}\left(U^{+}, \omega \mathscr{F}\right),\right.
$$

where $\tau$ is any choice of generalized Dehn twist supported in the interior of $T(\lambda)$ and we use the canonical identifications of $U^{-}, U^{+}$with $U$.

We denote the resulting foliated manifold by $\left(M^{L}, \mathscr{F}^{L}\right)$.

Proposition 13. The foliation $\left(M^{L}, \mathscr{F}^{L}\right)$ admits calibrations $\omega^{L}$. If $n>1$, then

(i) $\left(M^{L}, \mathscr{F}^{L}, \omega^{L}\right)$ is unique up to equivalence,

(ii) $[\omega]$ is integral if and only if $\left[\omega^{L}\right]$ is integral,

(iii) $\pi_{i}\left(M^{L}\right) \cong \pi_{i}(M)$ and $H_{i}\left(M^{L} ; \mathbb{Z}\right) \cong H_{i}(M ; \mathbb{Z}), 0 \leq i \leq n-1$.

Proof. We restrict our attention to $U(\epsilon)$. After cutting $U(\epsilon)$ along $U$ and gluing back using the identification $\chi$ in (6), we obtain

$$
U^{L}(\epsilon):=U^{-}(\epsilon) \#_{\chi} U^{+}(\epsilon) \subset M^{L} .
$$

Since the flow of $R$ preserves both $\omega$ and the foliation, the restriction of $\omega$ to $U^{-}(\epsilon)$ and $U^{+}(\epsilon)$ defines closed 2-forms $\omega^{-}$and $\omega^{+}$independent of the coordinate $t$. When we glue $U^{-}$to $U^{+}$using $\chi$, since this map is a symplectomorphism the 2-forms $\omega^{-}$and $\omega^{+}$induce a 2-form $\omega_{\epsilon}^{L}$ on $U^{L}(\epsilon)$. Then

$$
\omega^{L}:= \begin{cases}\omega & \text { in } M^{L} \backslash U^{L}(\epsilon), \\ \omega_{\epsilon}^{L} & \text { in } U^{L}(\epsilon)\end{cases}
$$

is the desired closed 2-form.

The 2-calibrated structure we obtain is unique up to equivalence. Firstly, different identifications $\varphi:\left(U, \omega_{\mathscr{F}}\right) \rightarrow\left(T(\lambda), d \alpha_{\text {can }}\right)$ are related by a global Poisson diffeomorphism. The reason is the same as in the proof of the uniqueness statement of Theorem 10: $S^{n}$ is simply connected for $n>1$. Secondly, generalized Dehn twists are symplectically isotopic by an isotopy supported in a neighborhood of the sphere. Thirdly, changing the Reeb vector field amounts to a change of variable in the coordinate $t$, and this does not modify the construction.

The calibration is a real cohomology class determined by its values on closed 2chains (which by a theorem of Thom are always homologous to embedded surfaces). If $n>1$ the 2-chains can be homotoped to avoid the neighborhood $U(\epsilon)$ of the Lagrangian sphere $L$, where $\omega^{L}$ coincides with $\omega$. Hence the integrality of the 2-calibrated foliation is unaffected by the surgery.

The same general position arguments imply that maps from CW complexes of dimension less or equal than $n$ can be homotoped to miss $U(\epsilon)$. Therefore homology and homotopy groups up to dimension $n-1$ are unaffected by the surgery. 
Remark 14. A "framed" Lagrangian $n$-sphere [Seidel 2000] is a parametrized $n$-sphere up to isotopy and the action of $\mathrm{O}(n+1)$. Generalized Dehn twists associated to two parametrizations defining the same "framed" Lagrangian $n$-sphere are isotopic, the isotopy by symplectomorphisms supported in a compact neighborhood of the Lagrangian sphere (Remark 5.1 in [Seidel 2000] or the paragraph after Lemma 1.10 in [Seidel 2003]). Therefore generalized Dehn surgery is well-defined for "framed" Lagrangian spheres.

Remark 15. The flow of the local Reeb vector field $R$ can be used to displace the Lagrangian sphere $L$ to a new Lagrangian sphere $L^{\prime}$ inside a nearby leaf. It follows that $\left(M^{L}, \mathscr{F}^{L}, \omega^{L}\right)$ and $\left(M^{L^{\prime}}, \mathscr{F}_{F}^{L^{\prime}}, \omega^{L^{\prime}}\right)$ are equivalent.

If, instead of $\tau$, we use its inverse, we get a 2-calibrated foliation $\left(M^{L^{-}}, \mathscr{F}^{L^{-}}, \omega^{L^{-}}\right)$ referred to as negative generalized Dehn surgery along $L$; negative generalized Dehn surgery is generalized Dehn surgery for the opposite coorientation.

Generalized Dehn surgery along $L$ and negative generalized Dehn surgery along $L$ are inverse to each other.

4.1. Lagrangian surgery. Let $L \subset(M, \mathscr{F}, \omega)$ be a parametrized Lagrangian sphere. Let $v(L)$ denote a tubular neighborhood of $L$, and $\nu \mathscr{F}(L)$ a tubular neighborhood of $L$ inside the leaf containing $L$. The parametrized Lagrangian sphere $L$ carries a canonical framing $\mu_{L}$ : because $L$ is Lagrangian $\nu_{\mathscr{F}}(L) \cong T^{*} L$ and we deduce

$$
v(L) \cong v_{\mathscr{F}}(L) \oplus \underline{\mathbb{R}} \cong T^{*} S^{n} \oplus \underline{\mathbb{R}} \cong \underline{\mathbb{R}}_{\mid S^{n}}^{n+1},
$$

where in the last isomorphism in (7) a positive nowhere-vanishing section of $\underline{\mathbb{R}}_{\mid} S^{n}$ is sent to the outward normal unit vector field. Therefore $L$ determines up to diffeomorphism an elementary cobordism $Z$, which amounts to attaching a $(n+1)$ handle to the parametrized sphere $L$ with framing $\mu_{L}$ [Gompf and Stipsicz 1999, Chapter 4]. The boundary of the cobordism is $\partial Z=M \amalg M^{\mu_{L}}$.

This subsection addresses the construction of $\left(M^{\mu_{L}}, \mathscr{F}^{\mu_{L}}, \omega^{\mu_{L}}\right)$, a 2-calibrated foliation which extends $(M, \mathscr{F}, \omega)$ on the complement of a neighborhood of $L$ (the complement understood as a subset of both $M$ and $M^{\mu_{L}}$ ). We do it by using the relation between symplectic manifolds and cosymplectic foliations presented in Section 2: we have to endow the cobordism $Z$ with a symplectic form $\Omega$ - at least in a neighborhood of the $(n+1)$-handle - and a symplectic vector field $Y$ transverse to the boundary. This produces automatically a cosymplectic foliation on $\partial Z$, and that is how we obtain $\left(M^{\mu_{l}}, \mathscr{F}^{\mu_{L}}, \omega^{\mu_{L}}\right)$. Remark that our strategy is the same one used in contact geometry to show that surgeries along Legendrian spheres give rise to new contact manifolds [Weinstein 1991, third paragraph on page 242].

The elementary cobordism $Z$ is the result of gluing a $(n+1)$-handle to the trivial cobordism $P_{1}:=M \times[-\varepsilon, \varepsilon]$. We have to define symplectic structures and 
symplectic vector fields transverse to the boundary on both the trivial cobordism and the $(n+1)$-handle in a way that is compatible with the gluing.

We start with the trivial cobordism $P_{1}$ : by the coisotropic embedding [Gotay 1982] there is a unique choice of symplectic structure on $P_{1}$ which extends the given closed 2-form $\omega$ on $M \times\{0\}$. We now give a specific normal form for it which is convenient for the purpose of describing a compatible gluing with the $(n+1)$-handle: let us denote $H_{1}:=v(L)$. Since the gluing between the trivial cobordism and the $(n+1)$-handle occurs near $v(L)$, we can assume without loss of generality that $P_{1}=H_{1} \times[-\varepsilon, \varepsilon]$. Let $\left(\mathscr{F}_{1}, \omega_{1}\right)$ denote the restriction of $(\mathscr{F}, \omega)$ to $H_{1}$. We select $R_{1}$ a positive Reeb vector field on $H_{1}$ with dual (closed) defining 1 -form $\alpha_{1}\left(i_{R} \alpha_{1}=1, \operatorname{ker} \alpha_{1}=\mathscr{F}_{1}\right)$. We let $v$ be the coordinate on the interval $[-\varepsilon, \varepsilon]$, and we extend $\alpha_{1}$ and $\omega_{1}$ to $H_{1} \times[-\varepsilon, \varepsilon]$ independently of $v$.

On $P_{1}$, we define $\Omega_{1}:=\omega_{1}+d\left(v \alpha_{1}\right)$, which is a symplectic form provided $\varepsilon$ is small enough.

As symplectic vector field on $\left(P_{1}, \Omega_{1}\right)$ we take $Y_{1}:=\partial / \partial v$, which is transverse to $H \times\{-\varepsilon\}$ and $H \times\{\varepsilon\}$.

We let $P_{2}$ denote the $(n+1)$-handle. Before defining the symplectic form $\Omega_{2}$ and a symplectic vector field $Y_{2}$ on $\left(P_{2}, \Omega_{2}\right)$, we address the problem of gluing symplectic cobordisms.

Lemma 16 [Gotay 1982, Extension theorem]. Let $\left(P_{j}, \Omega_{j}\right), j=1,2$, be symplectic manifolds, $H_{j} \subset P_{j}$ hypersurfaces and $Y_{j}$ symplectic vector fields transverse to them, so that we have product structures $H_{j} \times[-\varepsilon, \varepsilon]$. Define

$$
\omega_{j}=\Omega_{j_{\mid H_{j}}}, \quad \alpha_{j}=i_{Y_{j}} \Omega_{j_{\mid H_{j}}}
$$

and $\mathscr{F}_{j}$ the foliation integrating $\operatorname{ker} \alpha_{j}, j=1,2$. Suppose that $\phi: H_{1} \rightarrow H_{2}$ is a diffeomorphism such that $\phi^{*} \omega_{2}=\omega_{1}$ and $\phi^{*} \alpha_{2}=\alpha_{1}\left(\right.$ and therefore $\left.\phi^{*} \mathscr{F}_{2}=\mathscr{F}_{1}\right)$. Then

$$
\phi \times I d:\left(H_{1} \times[-\varepsilon, \varepsilon], \Omega_{1}\right) \rightarrow\left(H_{2} \times[-\varepsilon, \varepsilon], \Omega_{2}\right)
$$

is a symplectomorphism (obviously compatible with the symplectic vector fields).

Lemma 16 is the analog of Proposition 4.2 in [Weinstein 1991].

In our specific situation of gluing near Lagrangian spheres, the amount of information needed to describe $\phi$ as in Lemma 16 is much smaller.

Corollary 17. Let $\left(P_{j}, \Omega_{j}, H_{j}, Y_{j}\right), j=1,2$, be as in Lemma 16 and assume further that $L_{j} \subset H_{j}$ are Lagrangian spheres and $P_{j}$ small tubular neighborhoods of $L_{j}$.

Let $\theta: L_{1} \rightarrow L_{2}$ be a diffeomorphism. Then $\theta$ extends to an isomorphism of tuples $\left(P_{1}, \Omega_{1}, H_{1}, Y_{1}\right) \rightarrow\left(P_{2}, \Omega_{2}, H_{2}, Y_{2}\right)$. 
Proof. The symplectic vector fields give rise by contraction to closed 1-forms defining the foliations, and therefore to Reeb vector fields. We extend $\theta$ to a symplectomorphism of neighborhoods of the spheres inside their leaves, and we further extend it to $\phi:\left(H_{1}, \alpha_{1}, \omega_{1}\right) \rightarrow\left(H_{2}, \alpha_{2}, \omega_{2}\right)$ by declaring it to be equivariant with respect to the Reeb flows. By construction $\phi$ is in the hypothesis of Lemma 16.

Notice that the only choice is the identification of the symplectic neighborhoods of $L_{j}, j=1,2$, inside their respective leaves.

4.1.1. The choice of symplectic form and symplectic vector field on the $(n+1)$ handle. Let $W$ be a neighborhood of $0 \in \mathbb{C}^{n+1}$. This neighborhood will contain our $(n+1)$-handle $P_{2}$.

Let us consider the complex Morse function

$$
\begin{aligned}
h: \mathbb{C}^{n+1} & \rightarrow \mathbb{C}, \\
\left(z_{1}, \ldots, z_{n+1}\right) & \mapsto z_{1}^{2}+\cdots+z_{n+1}^{2} .
\end{aligned}
$$

We take $\Omega_{2} \in \Omega^{2}(W)$ to be any symplectic form of type $(1,1)$ at the origin with respect to the standard complex structure of $\mathbb{C}^{n+1}$, and $Y_{2}$ to be the Hamiltonian vector field of $-\operatorname{Im} h$.

Let us explain the reason behind the choice of $\left(\Omega_{2}, Y_{2}\right)$. In the construction of the symplectic $(n+1)$-handle we have to reconcile several aspects:

The data $\left(P_{2}, \Omega_{2}, Y_{2}\right)$ has to determine the standard $(n+1)$-handle: if $\Omega_{2}=\Omega_{\mathbb{R}^{2 n+2}}$ then $Y_{2}$ is the gradient flow of $-\operatorname{Re} h$ with respect to the Euclidean metric, whose dynamics determine the standard $(n+1)$-handle. In Lemma 18 we are going to prove that for $\Omega_{2}$ of type $(1,1)$ at the origin, the Hamiltonian vector field $Y_{2}$ has a hyperbolic singularity at $0 \in \mathbb{C}^{n+1}$. Therefore the flow of $Y_{2}$ has both the right dynamical behavior to construct a standard $(n+1)$-handle about $0 \in \mathbb{C}^{n+1}$ and the right symplectic behavior.

The second aspect is that we want to define Lagrangian surgery along $L$ so that it becomes equivalent to generalized Dehn surgery. Generalized Dehn twists appear in our current setting as follows: the origin $0 \in \mathbb{C}^{n+1}$ is an isolated critical point for $h$. Let $h_{z}$ denote the fiber $h^{-1}(z) \cap W, z \in \mathbb{C}$, and let $\Omega$ be any closed 2-form on $W$ for which the fibers $h_{z}$ are symplectic. The annihilator with respect to $\Omega$ of the tangent space to the fibers is an Ehresmann connection for $h: W \backslash\{0\} \rightarrow \mathbb{C}$. Parallel transport over a path not containing the critical value $0 \in \mathbb{C}$ defines a symplectomorphism from the regular fiber over the starting point to the regular fiber over the ending point. Seidel proves [2003, Lemma 1.10 in Section 1.2] that for a certain choice of closed 2-form $\Omega_{\tau}$, which is Kähler near the origin, and for all $r \in \mathbb{R}^{>0} \subset \mathbb{C}$, parallel transport of the fiber $h_{r}$ over the boundary of the disk $\bar{D}(r) \subset \mathbb{C}$ counterclockwise is conjugated to a generalized Dehn twist supported in a given $T(\lambda)$. An argument using Taylor expansions shows that for symplectic forms 
of type $(1,1)$ at the origin, the fibers $h_{z}$ are symplectic near the origin, and therefore there is an associated symplectic parallel transport with respect to $\Omega_{2}$. Besides, symplectic parallel transport with respect to $\Omega_{2}$ can be connected to symplectic parallel transport with respect to $\Omega_{\tau}$. The upshot is that symplectic parallel transport over $\bar{D}(r) \subset \mathbb{C}$ counterclockwise with respect to $\Omega_{2}$ can be isotoped to a generalized Dehn twist, which is the property we need to prove the equivalence of generalized Dehn surgery and Lagrangian surgery.

The third aspect is that we need a flexible choice of symplectic form $\Omega_{2}$ on the $(n+1)$-handle, so the cobordisms naturally associated to Lefschetz pencil structures to be described in Section 5.3 can be identified with Lagrangian surgery.

In the next lemma we collect some useful properties of parallel transport with respect to forms of type $(1,1)$ at the origin:

Lemma 18. Let $\Omega \in \Omega^{2}(W)$ be a symplectic form of type $(1,1)$ at the origin. Let $Y \in \mathfrak{X}(W)$ be the Hamiltonian vector field of $-\operatorname{Im} h$ with respect to $\Omega$. Then:

(i) $Y$ is a section of $\operatorname{Ann}(Y)^{\Omega}$ which vanishes at $0 \in \mathbb{C}^{n+1}$.

(ii) $h_{*} Y(p)$ is a strictly negative multiple of $\partial / \partial x$, where $p \in W \backslash\{0\}, z=(x, y)$.

(iii) $Y$ has a nondegenerate singularity at the origin with $n+1$ positive eigenvalues and $n+1$ negative eigenvalues.

(iv) For each $r \in \mathbb{R} \backslash\{0\}$ we have Lagrangian spheres $\Sigma_{r} \subset h_{r}$ characterized as the set of points contracting into the critical point by the parallel transport over the segment $[0, r]$; the spheres come with a parametrization up to isotopy and the action of $\mathrm{O}(n+1)$ (they are "framed"). More generally, for each $z$ and $\gamma$ an embedded curve joining $z$ and the origin, the points in $h_{\gamma(0)}$ sent to the origin by parallel transport over $\gamma$ are a Lagrangian sphere $\Sigma_{\gamma(0)}$. Their construction depends smoothly on $\Omega$ and $\gamma$.

(v) For any embedded curve $\gamma$ through the origin parallel transport

$$
\rho_{\gamma}:\left(h_{\gamma(0)} \backslash \Sigma_{\gamma(0)}, \Omega\right) \rightarrow\left(h_{\gamma(1)} \backslash \Sigma_{\gamma(1)}, \Omega\right)
$$

is a symplectomorphism possibly not everywhere defined.

Proof. This is a generalization of [Seidel 2003, Lemma 1.13] for local symplectic forms which are of type $(1,1)$ at the origin; also - and very important for our applications - smooth dependence on the symplectic form and curve $\gamma \subset \mathbb{C}$ is proved.

Points (i) and (ii) are a straightforward calculation. Point (iii) is also elementary once we use Taylor expansions at the origin.

Point (iii) implies that $0 \in \mathbb{C}^{n+1}$ is a hyperbolic singular point for $Y$ (see [Palis and de Melo 1982] for basic theory on dynamical systems). Let $W^{s}(Y)$ denote the stable manifold. Point (ii) implies that $\left[0, r_{0}\right) \subset h\left(W^{s}(Y)\right)$ for some $r_{0}>0$, and that 
for any $r \in\left(0, r_{0}\right)$ the intersection $h_{r} \cap W^{s}(Y)$ is transverse. Since $\Sigma_{r}:=h_{r} \cap W^{s}(Y)$ is a hypersurface of $W^{s}(Y)$ transverse to $Y$, it is diffeomorphic to a sphere. More precisely, the stable manifold theorem gives a parametrization $\Psi^{\text {st }}: B^{n+1} \rightarrow W^{s}(Y)$ of a neighborhood of the origin inside $W^{S}(Y)$, which is unique up to isotopy and the action of $\mathrm{O}(n+1)$, the latter associated to the choice of an orthonormal basis of the tangent space of $W^{s}(Y)$ at the origin; such a parametrization induces a parametrization $l: S^{n} \rightarrow \Sigma_{r}$ unique up to isotopy and the action of $\mathrm{O}(n+1)$.

That $\Sigma_{r}$ is Lagrangian follows from point (ii), exactly as in the proof of Lemma 1.13 in [Seidel 2003].

The result for any other point $z$ and a curve $\gamma$ joining it to the origin follows from the previous ideas applied to the Hamiltonian of $-\operatorname{Im}(F \circ h)$, where $F: \mathbb{C} \rightarrow \mathbb{C}$ is a diffeomorphism fixing the origin which sends $\gamma$ to $[0, r]$, for some $r \in \mathbb{R} \backslash\{0\}$.

If $\Omega_{u}$ is a smooth family, the stable manifold theorem with parameters (the proof of Theorem 6.2 in [Palis and de Melo 1982], Chapter 2, is seen to depend smoothly on parameters) gives parametrizations $\Psi_{u}^{\text {st }}: B^{n+1} \rightarrow W^{s}\left(Y_{u}\right)$ of neighborhoods of 0 inside the corresponding stable manifolds. This induces a smooth family of parametrizations of the Lagrangian spheres $l_{u}: S^{n} \rightarrow \Sigma_{u, r}$.

Clearly there is also smooth dependence on the path $\gamma$ if we choose diffeomorphisms $F_{\gamma}: \mathbb{C} \rightarrow \mathbb{C}$ with such dependence.

Parallel transport is not defined for points in $h_{\gamma(0)}$ which converge to the singular point $0 \in \mathbb{C}^{n+1}$, which by definition are the Lagrangian sphere $\Sigma_{\gamma(0)}$. Parallel transport may send points of $h_{\gamma(0)} \backslash \Sigma_{\gamma(0)}$ away from $W$. For those points which do not leave $W$, which at least are those close enough to $\Sigma_{\gamma(0)}$, parallel transport is a well-known symplectomorphism, and this finishes the proof of the lemma.

4.1.2. The shape of the symplectic $(n+1)$-handle. A parametrized sphere $L \subset M$ together with a framing determine a diffeomorphism $\phi: H \rightarrow S^{n} \times \overline{B^{n+1}}(1)$, where $H$ is a compact neighborhood of $L$ and $S^{n} \times \overline{B^{n+1}}(1)$ is seen as a subset of the boundary of the standard $(n+1)$-handle

$$
\overline{B^{n+1}}(1) \times \overline{B^{n+1}}(1) \subset \mathbb{R}^{n+1} \times \mathbb{R}^{n+1}=\mathbb{C}^{n+1} .
$$

The diffeomorphism determines the manifold with corners $M \#_{\phi} \overline{B^{n+1}}(1) \times \overline{B^{n+1}}(1)$. A way to smooth the corners uses the gradient flow $Y$ of $-\operatorname{Re} h$ with respect to the Euclidean metric: let us consider a function

$$
f: H \backslash L \cong S^{n} \times \overline{B^{n+1}}(1) \backslash S^{n} \times\{0\} \rightarrow \mathbb{R}^{+}
$$

supported in the interior of $H$, and such that near the attaching sphere $L \cong S^{n} \times\{0\}$ its value is the time needed to flow from $H$ to a neighborhood of

$$
L^{\prime} \cong\{0\} \times S^{n} \subset \overline{B^{n+1}}(1) \times S^{n} .
$$




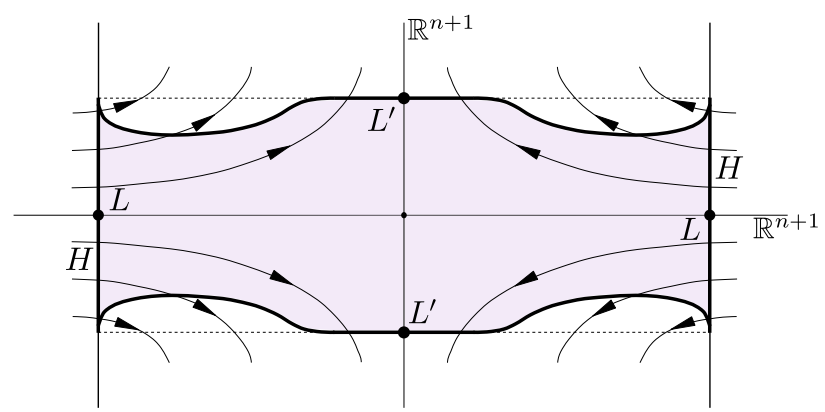

Figure 1. The modified handle is the shaded region, which is everywhere transverse to the gradient flow lines. The dotted segments are part of the boundary of the standard handle with corners.

Then $M^{\prime}=M \backslash H \cup \Phi_{1}^{f Y}(H \backslash L) \cup L^{\prime}$ is a smoothing of the new boundary of the cobordism. Actually, one equally thinks of using as modified handle the region bounded by $H$ and $\Phi_{1}^{f Y}(H \backslash L) \cup L^{\prime}$ (Figure 1).

We now proceed to define smoothings of the standard $(n+1)$-handle using $Y_{2}$, which is our symplectic replacement for the gradient flow of $-\operatorname{Re} h$. For the sake of flexibility in the definition of Lagrangian surgery we make the construction depend on a small enough parameter $r>0$.

We start by introducing some notation: the complex coordinate of $\mathbb{C}$ is $z=(x, y)$. For any $r, a, b \in \mathbb{R}$, we let $y_{r}(a, b), x_{r}(a, b) \subset \mathbb{C}$ be the "vertical" and "horizontal" segments joining the points $(r, a)$ and $(r, b)$, and $(a, r)$ and $(b, r)$ respectively.

Let us consider $r_{0}>0$ small enough so that the neighborhood $\left(W, \Omega_{2}\right)$ of $0 \in \mathbb{C}^{n+1}$ contains all Lagrangian spheres $\Sigma_{r}, r \in\left[-r_{0}, 0\right) \cup\left(0, r_{0}\right]$, described in point (iv) in Lemma 18. We fix $\epsilon>0$ small enough and define for all $r \in\left(0, r_{0}\right]$,

$$
H_{2, r}:=h^{-1}\left(y_{r}(-\epsilon, \epsilon)\right), \quad H_{2,-r}:=h^{-1}\left(y_{-r}(-\epsilon, \epsilon)\right) .
$$

By point (ii) in Lemma $18, Y_{2} \pitchfork H_{2, r}, H_{2,-r}$, so both hypersurfaces inherit 2calibrated foliations. By definition of the symplectic connection, the leaves of these 2-calibrated foliations are exactly the symplectic fibers of $h: H_{2, r} \rightarrow y_{r}(-\epsilon, \epsilon)$ and $h: H_{2,-r} \rightarrow y_{-r}(-\epsilon, \epsilon)$.

The Lagrangian sphere $\Sigma_{r}$ is going to be the attaching sphere of the $(n+1)$ handle, and therefore we need to specify an isotopy class of parametrizations (its framing is the Lagrangian framing): if $\Omega_{2}=\omega_{\mathbb{R}^{2 n+2}}=\sum_{i=1}^{n+1} d x_{i} \wedge d y_{i}$, then the Lagrangian sphere over $\left(r_{0}, 0\right)$ is the sphere radius $\sqrt{r_{0}}$ in the coordinates $x$ :

$$
\left\{(x, 0) \in \mathbb{R}^{2 n+2} \mid x_{1}^{2}+\cdots+x_{n+1}^{2}=r_{0}\right\} .
$$

Remark that the Lagrangian framing is the standard framing. The subset of forms of type $(1,1)$ at the origin is convex and hence connected (the symplectic condition 
holds for the segment close enough to the origin). We choose any path $\zeta$ connecting $\omega_{\mathbb{R}^{2 n+2}}$ to $\Omega_{2}$, and Lemma 18 with parameter space $\zeta$ allows us to transfer the canonical parametrization of the sphere of radius $\sqrt{r_{0}}$ to a parametrization $l$ of $\Sigma_{r_{0}}$. This completely determines the isotopy class of $l$.

To connect the hypersurfaces $H_{2, r}$ and $H_{2,-r}$ we want a careful parametrization of a neighborhood of $\Sigma_{r}$ inside $H_{2, r}, r \in\left(0, r_{0}\right]$. Let us extend the parametrization of $\Sigma_{r_{0}}$ to a neighborhood of $\Sigma_{r_{0}}$ inside its leaf

$$
\varphi_{r_{0}}:\left(U, \Omega_{\mathscr{F}}\right) \rightarrow\left(T(\lambda), d \alpha_{\text {can }}\right) .
$$

Parallel transport over the horizontal segment $x_{0}\left(r_{0}, r\right)$ induces a parametrization of a neighborhood of $\Sigma_{r}$ inside its leaf

$$
\varphi_{r}:=\varphi_{r_{0}} \circ \rho_{x_{0}\left(r, r_{0}\right)}:\left(\rho_{x_{0}\left(r, r_{0}\right)}^{-1}(U), \Omega_{\mathscr{F}}\right) \rightarrow\left(T(\lambda), d \alpha_{\text {can }}\right), r \in\left(0, r_{0}\right] .
$$

We define $T_{r}(\lambda):=\varphi_{r}^{-1}(T(\lambda)), r \in\left(0, r_{0}\right]$.

Let $R_{r}$ be the (negative) Reeb vector field on $H_{2, r}$ determined by the equality $h_{*} R_{r}=\partial / \partial y, r \in\left(0, r_{0}\right]$. The neighborhood of $\Sigma_{r}$ inside $H_{2, r}$ that we are going to consider is $T_{r}(\lambda, \epsilon)$, defined as in (5) using the flow of $R_{r}$ on $H_{2, r}$. In fact we redefine $H_{2, r}:=T_{r}(\lambda, \epsilon), r \in\left(0, r_{0}\right]$.

Let

$$
f_{r} \in C^{\infty}\left(T_{r}(\lambda, \epsilon) \backslash \Sigma_{r}, \mathbb{R}^{+}\right)
$$

have the following properties:

- The support of $f_{r}$ is contained in the interior of $T_{r}(\lambda, \epsilon)$.

- The time 1 flow of $f_{r} Y_{2}$ sends $T_{r}(\lambda / 2, \epsilon / 2) \backslash \Sigma_{r}$ into $H_{2,-r}$.

We use the hypersurface

$$
H_{2, r}^{\mu_{L}}:=\Phi_{1}^{f_{r} Y_{2}}\left(T_{r}(\lambda, \epsilon) \backslash \Sigma_{r}\right) \cup \Sigma_{-r}
$$

to define the handle $P_{2, r}$ as the compact domain of $\mathbb{C}^{n+1}$ bounded by $H_{2, r}^{\mu_{L}}$ and $H_{2, r}$. The new boundary of the cobordism is $M^{\mu_{L}}=\left(M \backslash H_{2, r}\right) \cup H_{2, r}^{\mu_{L}}$.

4.1.3. Lagrangian surgery.

Proposition 19. Any parametrized Lagrangian sphere $L \subset\left(M^{2 n+1}, \mathscr{F}_{P}, \omega\right), n>1$, determines symplectic elementary cobordisms $(Z, \Omega)$ carrying a symplectic vector field transverse to the boundary, which induce 2-calibrated foliations

$$
\left(M, \mathscr{F}_{F}, \omega\right),\left(M^{\mu_{L}}, \mathscr{F}^{\mu_{L}}, \omega^{\mu_{L}}\right) .
$$

Proof. Any form of type $(1,1)$ at the origin endows the $(n+1)$-handle $P_{2, r}$ with a symplectic structure $\Omega_{2}$. The Hamiltonian vector field $Y_{2}$ is transverse to $\partial P_{2, r}$ and determines a parametrized Lagrangian sphere $\Sigma_{r}$. The parametrized 
Lagrangian sphere $\Sigma_{r}$ with its Lagrangian framing is isotopic to the standard sphere with its standard framing. Therefore applying Corollary 17 produces the elementary cobordism $Z$. Moreover, it gives rise to a symplectic structure $\Omega$ and a symplectic vector field $Y$ transverse to $\partial Z$, which induce a 2-calibrated foliation on $\partial Z=M \amalg M^{\mu_{L}}$. By construction we recover $\left(\mathscr{F}_{F}, \omega\right)$ on $M$ and obtain $\left(\mathscr{F}^{\mu_{L}}, \omega^{\mu_{L}}\right)$ on $M^{\mu_{L}}$, which coincides with $(\mathscr{F}, \omega)$ away from a neighborhood of $L$.

Definition 20. Let $L \subset\left(M^{2 n+1}, \mathscr{F}, \omega\right), n>1$, be a parametrized Lagrangian sphere. We define Lagrangian surgery along $L$ as any of the 2-calibrated foliations $\left(M^{\mu_{L}}, \mathscr{F}^{\mu_{L}}, \omega^{\mu_{L}}\right)$ in Proposition 19, obtained as the new boundary component of the symplectic elementary cobordism, which amounts to attaching a symplectic $(n+1)$-handle as described in 4.1.1, 4.1.2, to the trivial symplectic cobordism determined by $(M, \mathscr{F}, \omega)$.

Remark 21. Instead of gluing the $(n+1)$-handle to the trivial cobordism, we can proceed the other way around. This amounts to reversing the coorientation on $(M, \mathscr{F}, \omega)$ and hence considering the opposite symplectic vector field $\operatorname{Im} h$ on the $(n+1)$-handle. Actually, we can do things in an equivalent way: on the $(2 n+2)$-dimensional $(n+1)$-handle we can use as attaching sphere $\Sigma_{-r}$ instead of $\Sigma_{r}, r>0$ (and also choosing an appropriate shape for the handle). We go from this second point of view to the first one by using the symplectic transformation $\left(z_{1}, \ldots, z_{n+1}\right) \mapsto\left(-i z_{1}, \ldots,-i z_{n+1}\right)$. It can be checked that the new boundary is a 2-calibrated foliation

$$
\left(M^{-\mu_{L}}, \mathscr{F}^{-\mu_{L}}, \omega^{-\mu_{L}}\right) .
$$

Surgery along $L$ with framing $\mu_{L^{-}}$gives (11) with opposite orientation.

4.1.4. Independence from choices. In the construction of $\left(M^{\mu_{L}}, \mathscr{F}^{\mu_{L}}, \omega^{\mu_{L}}\right)$ there are several choices both in the symplectic handle and in the trivial cobordism, which in principle may result into nonequivalent 2-calibrations $\omega^{\mu_{L}}$. The choices in the symplectic handle are the symplectic form $\Omega_{2}$, the parameter $r \in\left(0, r_{0}\right]$ ( $r_{0}$ itself depends on $\Omega_{2}$ ), the function $f_{r}$ (this includes the choice of $\epsilon>0$ ) and the parametrization $\varphi_{r_{0}}$. Choices in the trivial cobordism correspond to choices in $H_{1}$. There, we have a fixed $l^{-1}: L \rightarrow S^{n}$ and we choose an extension $\varphi$ : $\left(U, \omega_{\mathscr{F}}\right) \rightarrow\left(T(\lambda), d \alpha_{\text {can }}\right)$ and a Reeb vector field $R_{1}$. When applying Corollary 17 to construct the elementary cobordism $Z$, the choice of extension $\varphi_{r_{0}}$ is absorbed into the choice of extension $\varphi$.

In Theorem 26 we will show that for all $r>0$ small enough, Lagrangian surgery produces a 2-calibrated foliation equivalent to generalized Dehn surgery. Since according to Proposition 13 generalized Dehn surgery is independent of the extension $\varphi$ and of the Reeb vector field, we just need to prove independence of Lagrangian surgery on the function $f_{r}$ and the parameter $r$. Note that these 
two choices do not matter for the diffeomorphism type of $\left(M^{\mu_{L}}, \mathscr{F}^{\mu_{L}}\right)$. The key technical result that provides the required flexibility in our Poisson setting is an extension result for symplectomorphisms (Lemma 22).

Let us first address the case when all choices are the same except for the functions $f_{r}, f_{r}^{\prime}$ in (9). They give rise to two hypersurfaces $H_{2, r}^{\mu_{L}}\left(f_{r}\right), H_{2, r}^{\mu_{L}}\left(f_{r}^{\prime}\right)$ as described in (10), transverse to $Y_{2}$ and matching near their boundary and near $\Sigma_{-r}$. Following the flow lines of $Y_{2}$ defines a compactly supported diffeomorphism from $H_{2, r}^{\mu_{L}}\left(f_{r}\right)$ to $H_{2, r}^{\mu_{L}}\left(f_{r}^{\prime}\right)$. The diffeomorphism is a Poisson equivalence because, by construction, it is symplectic parallel transport over horizontal segments. Therefore the extension by the identity is a Poisson equivalence between the 2-calibrated foliations associated to $f_{r}$ and $f_{r}^{\prime}$. The general position argument used in the proof of Theorem 10 implies that this is in fact an equivalence of 2-calibrated foliations.

The case where the only different choice is $r<r^{\prime}$ is more delicate. We want to construct a Poisson equivalence

$$
\phi:\left(M^{\mu_{L}}, \mathscr{F}^{\mu_{L}}, \omega_{r}^{\mu_{L}}\right) \rightarrow\left(M^{\mu_{L}}, \mathscr{F}^{\mu_{L}}, \omega_{r^{\prime}}^{\mu_{L}}\right),
$$

which extends the identity map in the complement of $H_{2, r}^{\mu_{L}} \subset M^{\mu_{L}}$. Let us define $\phi_{1}: H_{2, r}^{\mu_{L}} \rightarrow H_{2, r^{\prime}}^{\mu_{L}}$ to be the map given by the flow lines of $Y_{2}$, which we just saw corresponds to symplectic parallel transport over horizontal segments. It is well defined near $\Sigma_{-r}$ because for points in $\Sigma_{-r} \subset H_{2, r}^{\mu_{L}}$ we make parallel transport over the segment $x_{0}\left(-r,-r^{\prime}\right)$, which does not contain the origin.

We need to introduce the following annular subsets around the Lagrangian sphere $\Sigma_{r}, r \in\left(0, r_{0}\right]:$

$$
\begin{aligned}
A_{r}\left(\lambda, \lambda^{\prime}\right) & :=T_{r}(\lambda) \backslash \operatorname{int} T_{r}\left(\lambda^{\prime}\right), & & \lambda>\lambda^{\prime}>0, \\
A_{r}\left(\lambda, \lambda^{\prime}, \epsilon, \epsilon^{\prime}\right) & :=T_{r}(\lambda, \epsilon) \backslash \operatorname{int} T_{r}\left(\lambda^{\prime}, \epsilon^{\prime}\right), & & \lambda>\lambda^{\prime}>0, \epsilon>\epsilon^{\prime}>0 .
\end{aligned}
$$

The boundary of an annular subset is made of an inner and an outer connected component, according to their distance to the Lagrangian sphere (Figure 2).

Let $\lambda^{\prime}, \epsilon^{\prime}>0$ be such that the supports of $f_{r}$ and $f_{r^{\prime}}$ do not intersect $A_{r}\left(\lambda, \lambda^{\prime}, \epsilon, \epsilon^{\prime}\right)$ and $A_{r^{\prime}}\left(\lambda, \lambda^{\prime}, \epsilon, \epsilon^{\prime}\right)$, respectively. Therefore $A_{r}\left(\lambda, \lambda^{\prime}, \epsilon, \epsilon^{\prime}\right) \subset H_{2, r}^{\mu_{L}} \cap H_{2, r}$ and $A_{r^{\prime}}\left(\lambda, \lambda^{\prime}, \epsilon, \epsilon^{\prime}\right) \subset H_{2, r^{\prime}}^{\mu_{L}} \cap H_{2, r^{\prime}}$, and on $A_{r}\left(\lambda, \lambda^{\prime}, \epsilon, \epsilon\right)$,

$$
\phi_{1}(p)=\rho_{x_{y(h(p))}}\left(r, r^{\prime}\right)(p) .
$$

Note that $\phi_{1}$ does not extend to the identity map on

$$
M^{\mu_{L}} \backslash T_{r}\left(\lambda^{\prime}, \epsilon^{\prime}\right) \subset M \rightarrow M^{\mu_{L}} \backslash T_{r}\left(\lambda^{\prime}, \epsilon^{\prime}\right) \subset M .
$$

The problem is that according to the parametrizations of $T_{r}(\lambda, \epsilon)$ and $T_{r^{\prime}}(\lambda, \epsilon)$ described in the paragraph following (8), the identity map corresponds to

$$
\phi_{2}(p):=\rho_{y_{r^{\prime}}(0, y(h(p))} \circ \rho_{x_{0}\left(r, r^{\prime}\right)} \circ \rho_{y_{r}(y(h(p)), 0)}(p) .
$$




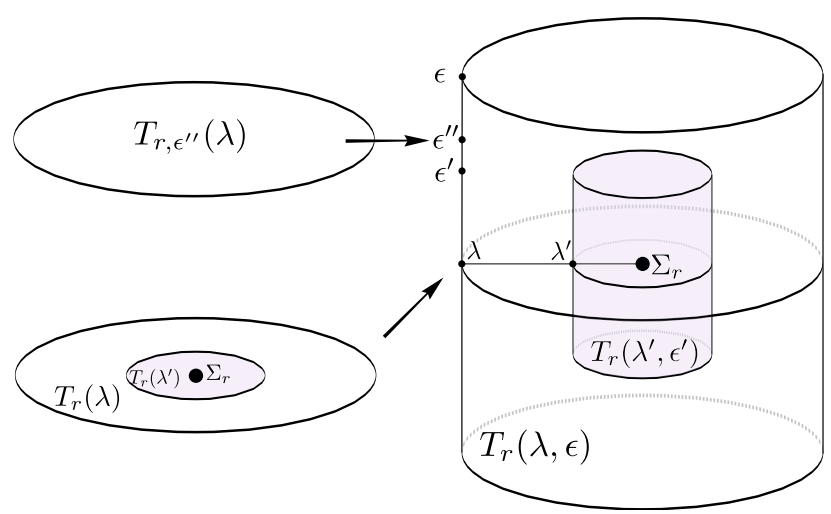

Figure 2. Right: the neighborhoods $T_{r}(\lambda, \epsilon)$ and $T_{r}\left(\lambda^{\prime}, \epsilon^{\prime}\right)$ of the Lagrangian sphere $\Sigma_{r}$. Horizontal slices correspond to intersections with leaves of the foliation. Left: the slice $t=0$, which intersects both $T_{r}(\lambda, \epsilon)$ and $T_{r}\left(\lambda^{\prime}, \epsilon^{\prime}\right)$, and the slice $t=\epsilon^{\prime \prime}$, which does not intersect $T_{r}\left(\lambda^{\prime}, \epsilon^{\prime}\right)$.

In addition, $\phi_{1}$ may not be everywhere defined since $\phi_{1}\left(A_{r}\left(\lambda, \lambda^{\prime}, \epsilon, \epsilon\right)\right)$ can fail to be contained in $A_{r^{\prime}}\left(\lambda, \lambda^{\prime}, \epsilon, \epsilon^{\prime}\right) \subset H_{2, r^{\prime}} \cap H_{2, r}^{\mu_{L}} \subset M^{\mu_{L}}$.

Let us assume the existence of $\left[\lambda_{1}, \lambda_{1}^{\prime}\right] \subset\left[\lambda, \lambda^{\prime}\right]$ and

$$
\phi_{3}: A_{r}\left(\lambda_{1}, \lambda_{1}^{\prime}, \epsilon, \epsilon^{\prime}\right) \rightarrow A_{r^{\prime}}\left(\lambda, \lambda^{\prime}, \epsilon, \epsilon^{\prime}\right)
$$

a Poisson diffeomorphism onto its image, which equals $\phi_{1}$ near the inner boundary of $A_{r}\left(\lambda_{1}, \lambda_{1}^{\prime}, \epsilon, \epsilon^{\prime}\right)$ and $\phi_{2}$ near the outer boundary. Then

$$
\phi:= \begin{cases}\phi_{1} & \text { in } H_{2, r}^{\mu_{L}} \backslash A_{r}\left(\lambda_{1}, \lambda_{1}^{\prime}, \epsilon, \epsilon^{\prime}\right), \\ \phi_{3} & \text { in } A_{r}\left(\lambda_{1}, \lambda_{1}^{\prime}, \epsilon, \epsilon^{\prime}\right), \\ \text { Id } & \text { in } M^{\mu_{L}} \backslash\left(H_{2, r}^{\mu_{L}} \cap T_{r}\left(\lambda_{1}, \epsilon\right)\right),\end{cases}
$$

is clearly an equivalence between $\left(M^{\mu_{L}}, \mathscr{F}^{\mu_{L}}, \omega_{r}^{\mu_{L}}\right)$ and $\left(M^{\mu_{L}}, \mathscr{F}^{\mu_{L}}, \omega_{r^{\prime}}^{\mu_{L}}\right)$.

The construction of $\phi_{3}$ requires the following basic result on extension of symplectic transformations, which is going to be also crucial to prove the equivalence of Lagrangian and generalized Dehn surgery.

Lemma 22. Let $\varsigma_{j}: A\left(\lambda, \lambda^{\prime}\right) \subset(T(\lambda), d \alpha) \rightarrow\left(T^{*} S^{n}, d \alpha\right), j=1,2, n>1$, be symplectic diffeomorphisms onto their image with the following properties:

(i) There exists $\left[\lambda_{1}, \lambda_{1}^{\prime}\right] \subset\left[\lambda, \lambda^{\prime}\right]$ such that $\sigma_{1}:=\varsigma_{2}^{-1} \circ \varsigma_{1}$ is defined on $A\left(\lambda_{1}, \lambda_{1}^{\prime}\right)$ and there exists $\sigma_{s}: A\left(\lambda_{1}, \lambda_{1}^{\prime}\right) \rightarrow\left(T^{*} S^{n}, d \alpha\right), s \in[0,1]$, an isotopy connecting the identity to $\sigma_{1}$ and satisfying $\sigma_{s}\left(A\left(\lambda_{1}, \lambda_{1}^{\prime}\right)\right) \subset A\left(\lambda, \lambda^{\prime}\right)$ for all $s \in[0,1]$.

(ii) The isotopy $\sigma_{s}$ is Hamiltonian. 
Then there exists $\varsigma:\left(A\left(\lambda, \lambda^{\prime}\right), d \alpha\right) \rightarrow\left(T^{*} S^{n}, d \alpha\right)$ a symplectic diffeomorphism onto its image, which coincides with $\varsigma_{1}$ and $\varsigma_{2}$, respectively, near the inner and outer boundaries of $A\left(\lambda, \lambda^{\prime}\right)$; moreover, if the $C^{0}$-norm of $\sigma_{s}$ is small enough, then $\varsigma$ sends $A\left(\lambda_{1}, \lambda_{1}^{\prime}\right)$ into $A(\lambda, \lambda)$.

In case $\varsigma_{j}, j=1,2$, the radii $\lambda, \lambda^{\prime}$, the isotopy $\sigma_{s}$ and the symplectic form $d \alpha$ depend on a smooth parameter, $\varsigma$ can be arranged to depend smoothly on the parameter.

Proof. Let us define

$$
V=\bigcup_{s \in[0,1]} \sigma_{s}\left(A\left(\lambda_{1}, \lambda_{1}^{\prime}\right)\right) \times\{s\} \subset T^{*} S^{n} \times[0,1] .
$$

Its inner and outer boundaries are by definition the union of the inner and outer boundaries, respectively, of $\sigma_{s}\left(A\left(\lambda_{1}, \lambda_{1}^{\prime}\right)\right)$.

Condition (i) implies $V \subset A\left(\lambda, \lambda^{\prime}\right) \times[0,1]$. Let $X$ be the vector field on $V$ whose flow after projection on $T^{*} S^{n} \times\{0\}$ gives the isotopy $\sigma_{s}$. Let $\beta_{s}=i_{X_{s}} d \alpha$. Since $\sigma_{s}$ is Hamiltonian there exists a (time dependent) Hamiltonian $F \in C^{\infty}(V)$ such that $d F_{s}=\beta_{s}$ and $F_{0}=0$.

Because $\sigma_{s}$ in an isotopy, for each $s \in[0,1]$ the subset $A\left(\lambda, \lambda^{\prime}\right) \backslash \sigma_{s}\left(A\left(\lambda_{1}, \lambda_{1}^{\prime}\right)\right)$ has an outer connected component $C_{o, s}$ (containing the outer boundary of $A\left(\lambda, \lambda^{\prime}\right)$ ) and an inner connected component $C_{i, s}$. We define

$$
\tilde{V}=\bigcup_{s \in[0,1]}\left(\sigma_{s}\left(A\left(\lambda_{1}, \lambda_{1}^{\prime}\right)\right) \cup C_{o, s}\right) \times\{s\} \subset A\left(\lambda, \lambda^{\prime}\right) \times[0,1] .
$$

Let $\tilde{F} \in C^{\infty}(\tilde{V})$ be a function which coincides with $F$ near the inner boundary of $V$ is supported inside $V$ and vanishes for $s=0$. Then the time 1 flow of the path of Hamiltonian vector fields of $\tilde{F}$ composed with $\varsigma_{2}$ is a symplectomorphism that coincides with $\varsigma_{1}$ and $\varsigma_{2}$, respectively, near the inner and outer boundaries of $A\left(\lambda, \lambda_{1}^{\prime}\right)$. The lemma is proved once we extend the symplectomorphism to $A\left(\lambda, \lambda^{\prime}\right)$ by using $\varsigma_{1}$ on $A\left(\lambda_{1}^{\prime}, \lambda^{\prime}\right)$.

Also, if the $C^{0}$-norm of the isotopy is arbitrarily small, we can pick $\hat{\lambda}_{1}<\lambda_{1}$ so that $\sigma_{s}\left(A\left(\hat{\lambda}_{1}, \lambda_{1}^{\prime}\right)\right) \subset A\left(\lambda_{1}, \lambda^{\prime}\right)$, and therefore $\varsigma\left(A\left(\lambda_{1}, \lambda_{1}^{\prime}\right)\right) \subset A\left(\lambda, \lambda^{\prime}\right)$.

Remark 23. There is an analogous symplectic extension result when the $\varsigma_{j}$, $j=1,2$, are defined on $T(\lambda)$. Under assumption (i) (with domain $T\left(\lambda_{1}\right)$ instead of $\left.A\left(\lambda_{1}, \lambda_{1}^{\prime}\right)\right)$, the outcome is $\varsigma$ is a symplectomorphism that matches $\varsigma_{1}$ in a neighborhood of $T\left(\lambda^{\prime}\right)$ and $\varsigma_{2}$ near the boundary of $T(\lambda)$. If the $C^{0}$-norm of the isotopy is small enough, then we can assume as well that $\varsigma\left(T\left(\lambda_{1}\right)\right) \subset T(\lambda)$.

We are going to apply Lemma 22 in several instances in which the isotopy $\sigma_{s}$ is defined by symplectic parallel transport over curves $\gamma_{s}$. To that end, we are going to recall a straightforward result to control the $C^{0}$-norm of $\sigma_{s}$. Before that we need to 
introduce some notation. Given curves $\gamma_{1}, \ldots, \gamma_{n} \subset \mathbb{C}$ parametrized by the interval and such that $\gamma_{l}(1)=\gamma_{l+1}(0), l=1, \ldots, n-1$, their concatenation is the piecewise smooth curve

$$
\gamma_{1} * \cdots * \gamma_{n}, \quad v \in[(l-1) / n, l / n] \mapsto \gamma_{l}(n(v-(l-1) / n)), \quad l=1, \ldots, n-1 .
$$

If we speak of a family of piecewise smooth curves, it is understood that all the curves can be written as a concatenation of the same number of curves and the family is smooth on each of the intervals.

Once we have fixed a symplectic form $\Omega$ on a neighborhood $W$ of the origin which makes the fibers of the quadratic form $h$ symplectic, any piecewise smooth curve $\gamma \subset \mathbb{C}$ inside the image of $h$ induces by parallel transport a symplectomorphism $\rho_{\gamma}$, which in general is not everywhere defined on $h_{\gamma(0)}$ (both for points converging to the critical points and for points escaping $W)$ : we just need to pull back the symplectic fibration $f:(W \backslash\{0\}, \Omega) \rightarrow \mathbb{C} \backslash\{0\}$ and follow over each smooth piece of the curve the 1-dimensional kernel of the closed 2-form induced on the pullback fibration. From now on, unless otherwise stated, by a curve $\gamma \subset \mathbb{C}$ we will mean a piecewise smooth curve such that on each smooth interval it is either constant or embedded. In this way (i) we can define horizontal lifts of $\gamma$ without using pullback bundles, and (ii) on each smooth interval $\gamma$ is the integral curve of a locally defined vector field. These two properties will make our proofs more transparent.

We also recall that $A_{r, t}\left(\lambda, \lambda^{\prime}\right), r \in\left(0, r_{0}\right], t \in\left[-\epsilon, \epsilon^{\prime}\right]$, stands for the time $t$ Reeb flow of $A_{r}\left(\lambda, \lambda^{\prime}\right)$, where the Reeb vector field is $R_{r}$. If we let $\tilde{Y}$ denote the horizontal lift of $\partial / \partial y$, then $R_{r}=\tilde{Y}$. Then we also define $A_{0, t}\left(\lambda, \lambda^{\prime}\right):=\Phi_{t}^{\tilde{Y}}\left(A_{0}\left(\lambda, \lambda^{\prime}\right)\right)$ $\left(A_{0}\left(\lambda, \lambda^{\prime}\right)\right.$ itself well defined because $A_{r_{0}}\left(\lambda, \lambda^{\prime}\right) \cap \Sigma_{r_{0}}$ is empty).

Lemma 24. Let $\kappa_{t, s} \subset \mathbb{C}, t \in\left[\delta, \delta^{\prime}\right], s \in[0,1]$, be a family of loops. Let $\gamma_{t, s, l}$ be a sequence of families of loops converging to $\kappa_{t, s}$ in the $C^{1}$-norm uniformly on $t, s$. If the horizontal lifts $\tilde{\kappa}_{t, s}$ starting at $A_{r, t}\left(\lambda, \lambda^{\prime}\right)$ are defined for all $v \in[0,1]$ (the lift neither converges to $0 \in \mathbb{C}^{n+1}$ nor leaves $W$ ), then the following hold:

(i) As l tends to infinity we have convergence $\rho_{\gamma_{t, s, l}} \stackrel{C^{0}}{\rightarrow} \rho_{\kappa_{t, s}}$ on $A_{r, t}\left(\lambda, \lambda^{\prime}\right)$ uniformly on $t, s$.

(ii) For any fixed $t$, if $\rho_{\kappa_{t, 0}}, \rho_{\gamma_{t, 0, l}}$ are the identity map, $\gamma_{t, s, l}$ does not intersect the origin and the homotopies $\gamma_{t, s, l}$ converge to the homotopy $\kappa_{t, s}$ in the $C^{2}$-norm, then $\rho_{\kappa_{t, s}}$ is a Hamiltonian isotopy.

Proof. Recall that $\rho_{\kappa_{t, s}}=\tilde{\kappa}_{t, s}(1)$. Let $K$ be the union of the horizontal lifts $\tilde{\kappa}_{t, s}$ starting at all $p \in A_{r, t}\left(\lambda, \lambda^{\prime}\right)$ for all $t, s$. By assumption $K \subset W$ is a compact subset not containing the critical point $0 \in \mathbb{C}$. Then we can work inside $U_{K} \subset W$ a compact neighborhood of $K$ missing the critical point, where the convergence in point (i) follows from basic ODE theory. 
If $n=2$ then $\kappa_{t, s}$ may not be Hamiltonian because $A_{r, t}\left(\lambda, \lambda^{\prime}\right)$ has nontrivial first Betti number. If $\gamma_{t, s, l}$ does not contain the origin, then parallel transport cannot converge to the critical point $0 \in \mathbb{C}^{n+1}$. It cannot scape $W$ for connectivity reasons: for each fixed $t$ and for $l$ large enough, parallel transport $\rho_{t, s . l}, s \in[0,1]$ is an isotopy sending $A_{r, t}\left(\lambda, \lambda^{\prime}\right)$ inside $h_{\kappa_{t, s}(0)} \cap W$. Then it must send $T_{r, t}(\lambda)$ inside $h_{\kappa_{t, s}(0)} \cap W$.

Because $T_{r, t}(\lambda)$ has trivial first Betti number, $\rho_{\gamma_{t, s, l}}$ is a Hamiltonian isotopy. Because convergence of the homotopies in the $C^{2}$-norm implies convergence of the isotopies in the $C^{1}$-norm, the closed 1 -form $\beta_{s}$ associated to the isotopy $\rho_{\kappa_{t, s}}$, $s \in[0,1]$, can be $C^{0}$-approximated by exact ones, and therefore it is exact and $\rho_{\kappa_{t, s}}$ is Hamiltonian.

Remark 25. A similar convergence result holds if the horizontal lifts start at all points in $T_{r, t}(\lambda)$.

We are ready to construct $\phi_{3}$ on $A\left(\lambda_{1}, \lambda_{1}^{\prime}, \epsilon, \epsilon^{\prime}\right)$, which coincides with the Poisson morphism $\phi_{1}$ of (12) near the inner boundary of $A\left(\lambda_{1}, \lambda_{1}^{\prime}, \epsilon, \epsilon^{\prime}\right)$ and with the morphism $\phi_{2}$ of (13) near the outer boundary.

Recall that $t$ is the coordinate on the interval $[-\epsilon, \epsilon]$ and fix $\epsilon^{\prime \prime} \in\left(\epsilon^{\prime}, \epsilon\right)$. In a first stage we are going to apply Lemma 22 to the restrictions to the $t$-leaf $\phi_{1, t}, \phi_{2, t}$ with parameter space $t \in\left[-\epsilon^{\prime \prime}, \epsilon^{\prime \prime}\right]$ : let us define

$$
\gamma_{t, 1}:=x_{t}\left(r, r^{\prime}\right) * y_{r^{\prime}}(t, 0) * x_{0}\left(r^{\prime}, r\right) * y_{r}(0, t) .
$$

By equations (12) and (13), $\sigma_{t}:=\phi_{2, t}^{-1} \circ \phi_{1, t}=\rho_{\gamma_{t, 1}}$. We let $\sigma_{t, s}:=\rho_{\gamma_{t, s}}$, where $\gamma_{t, s}$ is a family of curves in $\mathbb{C}$ connecting the constant path $(r, t)$ to $\gamma_{t, 1}$, for example as depicted in Figure 3.

To get control on the $C^{0}$-norm of $\rho_{\gamma_{t, s}}$, we define

$$
\kappa_{t, s}=y_{r}(t,(s-1) t) * y_{r}((s-1) t, t),
$$

and we let the family $\gamma_{t, s}$ vary with $r^{\prime}$, so that when $r^{\prime}$ converges to $r$ the curves $\gamma_{t, s}$ converge to the curves $\kappa_{t, s}$ in the $C^{1}$-norm. Since $\kappa_{t, s}$ does not contain the

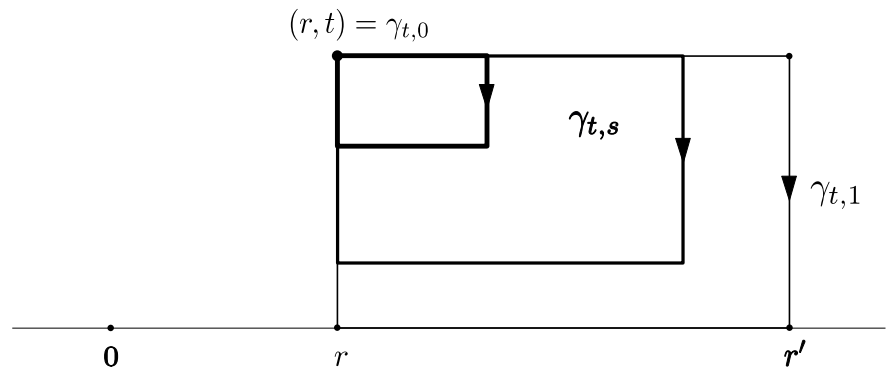

Figure 3. A family of curves shrinking $\gamma_{t, 1}$ the boundary of the rectangle to the vertex $(r, t)$. 
origin and $\rho_{\kappa_{t, s}}=\mathrm{Id}$, by Lemma 24 if $r^{\prime}$ is close enough to $r$ then $\rho_{\gamma_{t, s}}$ is as close as desired to the identity on $A_{r, t}\left(\lambda^{\prime}, \lambda\right)$ in the $C^{0}$-norm. Remark that here we do not use the full power of Lemma 24 , as the curves $\kappa_{t, s}$ do not contain the origin.

The conclusion is that for $t \in\left[-\epsilon^{\prime}, \epsilon^{\prime}\right]$, hypothesis (i) in Lemma 22 is satisfied (it is understood that we conjugate the isotopy problem in $A_{r, t}\left(\lambda, \lambda^{\prime}\right)$ to an isotopy problem in $A\left(\lambda, \lambda^{\prime}\right)$, using minus the Reeb flow for time $t$ and the chart $\left.\varphi_{r}\right)$. We can perform exactly the same construction for $|t| \in\left[\epsilon^{\prime}, \epsilon^{\prime \prime}\right]$ with the maps $\rho_{\kappa_{t, s}}=\mathrm{Id}, \rho_{\gamma_{t, s}}$ defined now on $T_{r, t}(\lambda)$, and conclude that the hypothesis of Remark 25 is also satisfied.

Because $\kappa_{s, t}$ does not contain $0 \in \mathbb{C}$, for $r^{\prime}$ close enough to $r$ the isotopy $\gamma_{t, s}$ misses the origin, and therefore it is a Hamiltonian isotopy. Thus the hypotheses of Lemma 22 and Remark 23 are satisfied. Inspection of the proof of Remark 23 shows that the lemma and remark can be combined to produce $\phi_{3, t}, t \in\left[-\epsilon^{\prime \prime}, \epsilon^{\prime \prime}\right]$, depending smoothly on $t$ and extending $\phi_{1}$ and $\phi_{2}$.

The extension for $|t| \in\left[\epsilon^{\prime \prime}, \epsilon\right]$ is straightforward: we let $\tilde{\sigma}_{t, s}$ be the isotopy corresponding to the Hamiltonian $\tilde{F}_{t}$ in the proof of Lemma 22 (rather in the proof of Remark 23). We have defined $\phi_{3, t}:=\phi_{2, t} \circ \tilde{\sigma}_{t, 1}$. Let $\beta:\left[\epsilon^{\prime \prime}, \epsilon\right] \rightarrow[0,1]$ be orientation-reversing and constant near the boundary. For $t \in\left[\epsilon^{\prime \prime}, \epsilon\right]$ we set $\phi_{3, t}:=\phi_{2, t} \circ \sigma_{\epsilon^{\prime \prime}, \beta(s)}$. For negative $t$ we proceed analogously and this produces the required extension $\phi_{3, t}, t \in[-\epsilon, \epsilon]$.

We have showed that Lagrangian surgery produces equivalent 2-calibrations if $r, r^{\prime}$ are close enough, which obviously implies the independence of the construction on $r \in\left(0, r_{0}\right]$.

\subsection{Generalized Dehn surgery is equivalent to Lagrangian surgery. The equiv-} alence of the two surgeries will remove all dependences appearing in Lagrangian surgery. The proof of the equivalence bears much resemblance to the proof of the independence of Lagrangian surgery on the parameter $r>0$, though it has additional technical complications. We give a brief overview in the following paragraphs.

To construct the equivalence between $\left(M^{L}, \mathscr{F}^{L}, \omega^{L}\right)$ and $\left(M^{\mu_{L}}, \mathscr{F}^{\mu_{L}}, \omega^{\mu_{L}}\right)$, a Poisson diffeomorphism suffices. The morphism is defined to be the identity away from a neighborhood of the Lagrangian spheres; then - working already in the symplectic handle we used in the cobordism - following the flow lines of $Y_{2}$ extends the identity to a morphism

$$
\phi_{2}: H_{2, r} \backslash T_{r}(\lambda / 2, \epsilon / 2) \rightarrow H_{2, r}^{\mu_{L}} .
$$

For some $\lambda^{\prime} \in(\lambda / 2, \lambda), \epsilon^{\prime} \in(\epsilon / 2, \epsilon), \phi_{2}$ restricts in $A_{r}\left(\lambda^{\prime}, \lambda / 2, \epsilon^{\prime}, \epsilon / 2\right)$ to parallel transport over horizontal segments $x_{t}(r,-r)$.

Let us cut $T_{r}\left(\lambda^{\prime}, \epsilon^{\prime}\right)$ along $T_{r}\left(\lambda^{\prime}\right)$ and let $\chi: T_{r}^{+}\left(\lambda^{\prime}\right) \rightarrow T_{r}^{-}\left(\lambda^{\prime}\right)$ be conjugated to a generalized Dehn twist supported in the interior of $T(\lambda / 2)$. We would be done if perhaps after modifying $\phi_{2}$ near the inner boundary of $A_{r}\left(\lambda^{\prime}, \lambda / 2, \epsilon^{\prime}, \epsilon / 2\right)$, we can 
extend it to a morphism

$$
\phi_{3}: T_{r}^{+}\left(\lambda^{\prime}, \epsilon^{\prime}\right) \#_{\chi} T_{r}^{-}\left(\lambda^{\prime}, \epsilon^{\prime}\right) \rightarrow H_{2,-r} \subset H_{2, r}^{\mu_{L}} .
$$

Equivalently, we need a pair of morphisms $\phi_{3}^{ \pm}: T_{r}^{ \pm}\left(\lambda^{\prime}, \epsilon^{\prime}\right) \rightarrow H_{2,-r}$ which satisfy

$$
\phi_{3}^{+}(p)=\phi_{3}^{-} \circ \chi(p), p \in T_{r}^{+}\left(\lambda^{\prime}\right),
$$

and which are independent of $t$ for $|t|$ small, so the induced morphism $\phi_{3}^{+} \#_{\chi} \phi_{3}^{-}$is smooth.

If $\Omega_{2}$ is the closed 2-form $\Omega_{\tau}$, then $\chi$ can be taken to be $\rho_{\partial \bar{D}(r)}$, parallel transport over $\partial \bar{D}(r)$ counterclockwise.

Consider the positive half disks $\partial \bar{D}^{+}(r):=\left\{r e^{i \theta \pi} \mid 0 \leq \theta \leq 1\right\} \subset \mathbb{C}$, and set $\zeta_{t}=y_{r}(t, 0) * \partial \bar{D}^{+}(r) * y_{-r}(0, t)$. Then define

$$
\begin{aligned}
\rho^{+}: T_{r}^{+}\left(\lambda^{\prime}, \epsilon^{\prime}\right) & \rightarrow H_{2,-r}, \\
p & \mapsto \rho_{\zeta_{y(h(p))}}(p),
\end{aligned}
$$

and define $\rho^{-}$on $T_{r}^{-}\left(\lambda^{\prime}, \epsilon^{\prime}\right)$ by parallel transport over the reflection of $\zeta_{t}$ in the $x$-axis. Then $\rho^{ \pm}$satisfy (15) and therefore they induce a morphism as in (14). But this morphism does not match $\phi_{2}$ because for the latter we do parallel transport over horizontal segments and for $\rho^{ \pm}$we use half disks (up to composition with vertical segments). So our problem reduces to define Poisson equivalences on $A_{r}^{ \pm}\left(\lambda^{\prime}, \lambda / 2, \epsilon^{\prime}, \epsilon / 2\right)$, which extend parallel transport over $\zeta_{t}$ (and its reflection in the $x$-axis) near the inner boundary and parallel transport over $x_{t}(-r, r)$ near the outer boundary. Of course, the extensions $\phi_{3}^{ \pm}$have to be compatible on $A_{r}^{ \pm}\left(\lambda^{\prime}, \lambda / 2\right)$ with $\chi$; because $\chi$ is supported in the interior of $T_{r}(\lambda / 2)$ the extensions must coincide on $A_{r}^{ \pm}\left(\lambda^{\prime}, \lambda / 2\right)$. This compatibility condition is going to follow from a careful choice of the families of curves connecting $x_{r}(-t, t)$ to $\zeta_{t}$. Since we will be doing parallel transport near the critical point, we will need the full power of Lemma 24 to argue that we can control the norm of the isotopies we construct and hence we are in the hypothesis of the interpolation lemma.

A further technical complication appears because the symplectic form $\Omega_{2}$ in the handle is different from $\Omega_{\tau}$. So the extension of parallel transport over segments and half disks has to include a deformation from parallel transport with respect to $\Omega_{2}$ to parallel transport with respect to $\Omega_{\tau}$.

Theorem 26. Assume $n>1$. We have equivalences of 2-calibrated foliations

$$
\phi:\left(M^{L}, \mathscr{F}^{L}, \omega^{L}\right) \rightarrow\left(M^{\mu_{L}}, \mathscr{F}^{\mu_{L}}, \omega^{\mu_{L}}\right)
$$

for all $r>0$ small enough. 
Proof. Stage 1. The complement $(M, \mathscr{F}, \omega) \backslash T_{r}(\lambda, \epsilon)$ can be seen as a subset of both $(M, \mathscr{F}, \omega)$ and $\left(M^{\mu_{L}}, \mathscr{F}^{\mu_{L}}, \omega^{\mu_{L}}\right)$. We may assume without loss of generality that for some $\lambda^{\prime}>\lambda / 2, \epsilon^{\prime}>\epsilon / 2$, the time 1 flow of $f_{r} Y_{2}$ sends $T_{r}\left(\lambda^{\prime}, \epsilon^{\prime}\right) \backslash \Sigma_{r}$ into $H_{2,-r} \subset H_{2, r}^{\mu_{L}} \subset M^{\mu_{L}}$. We define

$$
\phi_{0}= \begin{cases}\text { Id } & \text { in } M \backslash T_{r}(\lambda, \epsilon), \\ \Phi_{1}^{f_{r} Y_{2}} & \text { in } A_{r}(\lambda, \lambda / 2, \epsilon, \epsilon / 2),\end{cases}
$$

which is a Poisson morphism given on $A_{r}\left(\lambda, \lambda / 2, \epsilon^{\prime}, \epsilon / 2\right)$ by parallel transport over horizontal segments $x_{t}(-r, r), t \in\left[-\epsilon^{\prime}, \epsilon^{\prime}\right]$.

Stage 2. In both $H_{2, r}$ and $H_{2,-r}$ we have Reeb vector fields $R_{r}, R_{-r}$ defined near $\Sigma_{r}$ and $\Sigma_{-r}$ respectively (they are horizontal lifts of $\partial / \partial y$ ). Their flow parametrizes the leaf spaces by $t \in[-\epsilon, \epsilon]$. For the purpose of checking the smoothness of the morphism $\phi: M^{L} \rightarrow M^{\mu_{L}}$ in the statement of the theorem, in this stage we shall modify $\phi_{0}$ near the inner boundary of $A_{r}\left(\lambda^{\prime}, \lambda / 2, \epsilon^{\prime}, \epsilon / 2\right)$ to make it $t$-invariant for $|t|$ small (equivariant with respect to the flows of $R_{r}$ and $R_{-r}$ ).

Let $\beta:\left[-\epsilon^{\prime}, \epsilon^{\prime}\right] \rightarrow\left[-\epsilon^{\prime}, \epsilon^{\prime}\right]$ be an odd monotone function which is the identity near the boundary and maps to zero exactly the interval $[-\delta, \delta]$, with $0<\delta<\epsilon / 2$. Set

$$
\zeta_{t}:=y_{r}(t, \beta(t)) * x_{\beta(t)}(r,-r) * y_{-r}(\beta(t), t), \quad t \in\left[-\epsilon^{\prime}, \epsilon^{\prime}\right],
$$

and define

$$
\phi_{1}(p)=\rho_{\zeta_{y(h(p))}}(p), \quad p \in A_{r}\left(\lambda^{\prime}, \lambda / 2, \epsilon^{\prime}, \epsilon / 2\right),
$$

which by construction is $t$-invariant for $t \in[-\delta, \delta]$.

We are going to construct $\phi_{2}^{\prime}: A_{r}\left(\lambda^{\prime}, \lambda / 2, \epsilon^{\prime}, \epsilon / 2\right) \rightarrow H_{2,-r}$ extending $\phi_{0}$ near the outer boundary and $\phi_{1}$ near the inner boundary, by applying Lemma 22: let

$$
\begin{aligned}
& \gamma_{t, s}= \\
& y_{r}(t,(1-s) t+s \beta(t)) * x_{(1-s) t+s \beta(t)}(r,-r) * y_{-r}((1-s) t+s \beta(t), t) * x_{t}(-r, r),
\end{aligned}
$$

with $t \in\left[-\epsilon^{\prime}, \epsilon^{\prime}\right], s \in[0,1]$ and $r \in\left(0, r_{0}\right]$. Parallel transport over $\gamma_{t, s}$ defined on $A_{r, t}\left(\lambda^{\prime}, \lambda / 2\right)$ connects the identity map to $\phi_{0, t}^{-1} \circ \phi_{1, t}$. To estimate the $C^{0}$-norm of $\rho_{\gamma_{t, s}}$ we define $\kappa_{t, s}$ by using the formula of $\gamma_{t, s}$ in (17) for $r=0$, and consider $\rho_{\kappa_{t, s}}$ with domain $A_{0, t}\left(\lambda^{\prime}, \lambda / 2\right)$. By construction $\rho_{\kappa_{t, s}}$ is the identity.

Let $\gamma_{t, s}^{\prime}$ be the conjugation of $\gamma_{t, s}$ by $x_{t}(0, r)$ and let us consider $\rho_{\gamma_{t, s}^{\prime}}$ defined on $A_{0, t}\left(\lambda^{\prime}, \lambda / 2\right)$, the same domain as for $\kappa_{t, s}$.

We construct the extension $\phi_{2, t}^{\prime}$ first for the leaves in $[-\epsilon / 2, \epsilon / 2]$ : the union of the horizontal lifts $\tilde{\kappa}_{t, s}$ at $A_{0, t}\left(\lambda^{\prime}, \lambda / 2\right)$ is exactly

$$
K=\bigcup_{t \in[-\epsilon / 2, \epsilon / 2]} A_{0, t}\left(\lambda^{\prime}, \lambda / 2\right)
$$


a compact subset not containing the critical point $0 \in \mathbb{C}^{n+1}$. The curves $\gamma_{t, s}^{\prime}$ clearly converge in the $C^{1}$-norm to $\kappa_{t, s}$ as $r$ goes to zero. Therefore by point (i) in Lemma 24 there is $C^{0}$-convergence of $\rho_{\gamma_{t, s}^{\prime}}$ to the identity.

The same result holds for $\rho_{\gamma_{t, s}}$, though not automatically since parallel transport over $x_{t}(0, r)$ does not send $A_{0, t}\left(\lambda^{\prime}, \lambda / 2\right)$ diffeomorphically into $A_{r, t}\left(\lambda^{\prime}, \lambda / 2\right)$. This is the same situation as in the proof of independence of Lagrangian surgery on $r$. We define

$$
\tau_{t, s}=x_{t}(0, r) * y_{r}\left((t,(s-1) t) * x_{(s-1) t}(r, 0) * y_{r}((s-1) t, t) .\right.
$$

For $r=0$ we get $x_{t}(0,0) * y_{0}(t,(s-1) t) * x_{(s-1) t}(0,0) * y_{0}((s-1) t, t)$. We consider parallel transport $\rho_{\tau_{t, s}}$ defined on $A_{r, t}\left(\lambda^{\prime}, \lambda / 2\right)$, which for $r=0$ is the identity. Since for $r=0$ the union of the horizontal lifts of $\tau_{t, s}$ starting at $A_{0, t}\left(\lambda^{\prime}, \lambda / 2\right)$ is again $K$ in (18), by point (i) in Lemma 24 we conclude that $\rho_{x_{t}(0, r)}\left(A_{0, t}\left(\lambda^{\prime}, \lambda / 2\right)\right)$ converges to $A_{r, t}\left(\lambda^{\prime}, \lambda / 2\right)$ in the $C^{0}$-norm as $r$ tends to zero, and this finishes the proof of the estimate needed in point (i) of Lemma 22 for $t \in[-\epsilon / 2, \epsilon / 2]$.

For $|t| \in\left[\epsilon / 2, \epsilon^{\prime}\right]$ the estimate holds by connectivity arguments already mentioned: the proof above shows that for some interval $\left[\lambda_{1}^{\prime}, \lambda_{2}^{\prime}\right] \subset\left[\lambda^{\prime}, \lambda / 2\right]$, the isotopy $\rho_{\gamma_{t, s}}$ sends $A_{r, t}\left(\lambda_{1}^{\prime}, \lambda_{2}^{\prime}\right)$ into $A_{r, t}\left(\lambda^{\prime}, \lambda / 2\right)$, for $|t| \in\left[\epsilon / 2, \epsilon^{\prime}\right]$. Hence it must send $T_{r, t}\left(\lambda_{1}^{\prime}\right)$ into $T_{r, t}\left(\lambda^{\prime}\right)$.

The isotopies $\rho_{\gamma_{t, s}}$ are Hamiltonian: if $t$ is not in $\left[-\delta, \delta\right.$, ], then $\rho_{\gamma_{t, s}}$ extends to $T_{r, t}\left(\lambda^{\prime}\right)$ because $\gamma_{t, s}$ does not contain the origin. For the remaining values of $t$ it easy to check that the homotopy $\gamma_{t, s}$ can be approximated in the $C^{2}$-norm by a homotopy which does not contain $0 \in \mathbb{C}$. Therefore by point (ii) in Lemma 24 the isotopies are Hamiltonian. Hence we can apply Lemma 22 and Remark 23 in a compatible manner to produce $\phi_{2}^{\prime}$ on $A_{r}\left(\lambda^{\prime}, \lambda / 2, \epsilon^{\prime \prime} \epsilon / 2\right), \epsilon^{\prime \prime} \in\left(\epsilon / 2, \epsilon^{\prime}\right)$, extending $\phi_{0}$ and $\phi_{1}$. For the $t$-leaves with $|t| \in\left[\epsilon^{\prime \prime}, \epsilon^{\prime}\right]$, we apply the same patching trick as in the construction of the extension $\phi_{3}$ at the end of 4.1.4.

We define for $r>0$ small enough

$$
\phi_{2}= \begin{cases}\phi_{0} & \text { in } M \backslash T_{r}\left(\lambda^{\prime}, \epsilon^{\prime}\right), \\ \phi_{2}^{\prime} & \text { in } A_{r}\left(\lambda^{\prime}, \lambda / 2, \epsilon^{\prime}, \epsilon / 2\right),\end{cases}
$$

which is a Poisson morphism independent of $t \in[-\delta, \delta]$.

Stage 3. In this stage we cut $M$ along a neighborhood of $L$ inside its leaf $F_{L}$, and then define a Poisson morphism which extends $\phi_{2}$ from Stage 2 and parallel transport over boundaries of half disks ("conjugated" by vertical segments); the latter parallel transport also includes a deformation from $\Omega_{2}$ to $\Omega_{\tau}$.

Let us assume for the moment that $\Omega_{2}$ equals $\Omega_{\mathbb{R}^{2 n+2}}$. The closed 2-forms $\Omega_{\tau}$ [Seidel 2003, Section 1.2] are written $\Omega_{\tau}=\Omega_{\mathbb{R}^{2 n+2}}+d \alpha$, where $d \alpha$ vanishes on the tangent space to the fibers $h_{z}$ and is zero in a neighborhood of the union of the stable and the unstable manifold of $Y_{2}$ with respect to $\Omega_{\mathbb{R}^{2 n+2}}$. The first property implies 
that the fibers $h_{z}$ are symplectic. The second property implies that symplectic parallel transport with respect to $\Omega_{\tau}$ over $x_{0}(r,-r)$ is defined on $T_{r}(\lambda) \backslash \Sigma_{r}$.

We assume that $\alpha$ has been chosen so that parallel transport over $\partial \bar{D}(r)$ counterclockwise is conjugated by $\varphi_{r}$ to a generalized Dehn twist supported in the interior of $T(\lambda / 2)$. Let us define

$$
\Omega_{u}=\Omega_{\mathbb{R}^{2 n+2}}+u d \alpha, \quad u \in[0,1],
$$

and let $u:\left[0, \epsilon^{\prime}\right] \rightarrow[0,1]$ be a monotone function which attains the value 0 on $\left[2 \delta / 3, \epsilon^{\prime}\right]$ and the value 1 on $[0, \delta / 3]$.

Let us consider the arcs $\partial \bar{D}_{t}^{+}(r):=\left\{(0, t)+r e^{i \theta \pi}\right\} \subset \mathbb{C}$, and define the curves $\zeta_{t}=y_{r}(t, \beta(t)) * \partial \bar{D}_{\beta(t)}^{+}(r) * y_{-r}(\beta(t), t)$. Next we cut $T_{r}\left(\lambda^{\prime}, \epsilon^{\prime}\right)$ along $T_{r}\left(\lambda^{\prime}\right)$ and define on $T_{r}^{+}\left(\lambda^{\prime}, \epsilon^{\prime}\right)$

$$
\phi_{3}(p)=\rho_{u(y(h(p))), \zeta_{y(h(p))}(p),}
$$

which is $t$-invariant for $t \in[0, \delta / 3]$ and on $T_{r, 0}\left(\lambda^{\prime}\right)$ is parallel transport over $\partial \bar{D}(r)^{+}$ counterclockwise with respect to $\Omega_{\tau}$, and therefore conjugated to a Dehn twist supported in the interior of $T(\lambda / 2)$. We stress that this is a Poisson morphism because the restriction of $\Omega_{u}$ to fibers of $h$ is independent of $u$ (of course what changes is the symplectic connection).

We address now the construction of $\phi_{3}^{+}$on $A_{r}^{+}\left(\lambda^{\prime}, \lambda / 2, \epsilon^{\prime}, \epsilon / 2\right)$, a Poisson morphism extending $\phi_{2}$ and $\phi_{3}$ and $t$-invariant for $t \in[0, \delta / 3]$, using the same pattern as in Stage 2.

Let us define the curves

$$
\gamma_{t, s}=y_{r}(t, \beta(t)) * x_{\beta(t)}(r, s r) * \partial \bar{D}_{\beta(t)}^{+}(s r) * x_{\beta(t)}(-s r, r) * y_{r}(\beta(t), t),
$$

for $t \in\left[0, \epsilon^{\prime}\right]$ and $s \in[0,1]$ (see Figure 4). We have $\phi_{\gamma_{t, 1}}=\phi_{2, t}^{-1} \circ \phi_{3, t}, \phi_{\gamma_{t, 0}}=\mathrm{Id}$.

Smoothness of $\rho_{u(t), \gamma_{t, s}}$ for $s=0$ may not be evident.

Lemma 27. The map $\rho_{u(t), \gamma_{t, s}}$ depends smoothly on $t, s$.
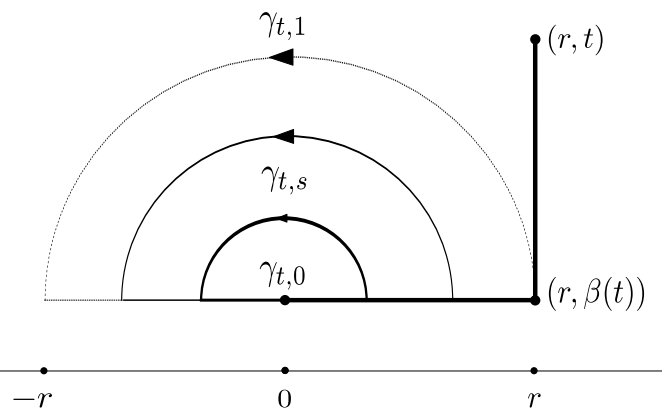

Figure 4. The curves $\gamma_{t, s}$ defined in (20). 
Proof. We rewrite $\rho_{u(t), \gamma_{t, s}}$ using vector fields on $\mathbb{C}$ whose integral curves are the pieces whose concatenation defines $\gamma_{t, s}$. Let

$$
X:=\frac{\partial}{\partial x}, \quad Y:=\frac{\partial}{\partial y}, \quad \Theta_{r, t}:=r x \frac{\partial}{\partial y}-(r(y-t)) \frac{\partial}{\partial x}, \quad t \in \mathbb{R},
$$

be vector fields on $\mathbb{C}$. Let $\tilde{X}_{u}, \tilde{Y}_{u}, \tilde{\Theta}_{u, r, t} \in \mathfrak{X}(W \backslash\{0\})$ be their horizontal lifts with respect to the symplectic connection defined by $\Omega_{u}$. The flows $\Phi_{l}^{\tilde{X}_{u}}, \Phi_{l}^{\tilde{Y}_{u}}, \varphi_{l}^{\tilde{\Theta}_{u, r, t}}$ are smooth in $u, r, t, l$. It follows that

$$
\rho_{u(t), \gamma_{t, s}}=\Phi_{t-\beta(t)}^{\tilde{Y}_{u(t)}} \circ \Phi_{(s+1) r}^{\tilde{X}_{u(t)}} \circ \Phi_{\pi}^{\tilde{\Theta}_{u(t), s r, \beta(t)}} \circ \Phi_{(1-s) r}^{-\tilde{X}_{u(t)}} \circ \Phi_{t-\beta(t)}^{-\tilde{Y}_{u(t)}},
$$

and thus $\rho_{u(t), \gamma_{t, s}}$ has smooth dependence on $t, s$.

The estimate in Lemma 24 is written for parallel transport with respect to a fixed symplectic form, but it can be checked that it holds true as well in case the parallel transport is with respect $\Omega_{u(t)}$. Let $\kappa_{t, s}$ be as defined in (17) for $r=0$. By comparing $\rho_{u(t), \gamma_{t, s}}$ with $\rho_{u(t), \kappa_{t, s}}=$ Id as in the previous stage (first conjugating with $x_{t}(r, 0)$ to have common domain, and then showing that the estimate holds after undoing the conjugation), we get control on the $C^{0}$-norm of $\rho_{u(t), \gamma_{t, s}}$ for $r$ small enough. The isotopies $\rho_{u(t), \gamma_{t, s}}$ are Hamiltonian since they can be $C^{1}$-approximated by Hamiltonian ones. Given that the isotopy $\rho_{u(t), \gamma_{t, s}}$ is $t$-invariant for $t \in[0, \delta / 3]$, choices in the proof of Lemma 22 can be done to obtain, for all $r$ small enough, an extension $\phi_{3}^{+}$on $A_{r}^{+}\left(\lambda^{\prime}, \lambda / 2, \epsilon^{\prime}, \epsilon / 2\right)$ which is $t$-invariant for $t \in[0, \delta / 3]$.

For $t \in\left[-\epsilon^{\prime}, 0\right]$ we proceed as we did for positive values, but using the reflection of the curves $\gamma_{t, s}$ in the $x$-axis. It is possible to arrange the proof of Lemma 22 to produce an extension $\phi_{3}^{-}$on $A_{r}^{+}\left(\lambda^{\prime}, \lambda / 2, \epsilon^{\prime}, \epsilon / 2\right)$ such that

- $\phi_{3}^{-}$is $t$-invariant for $t \in[-\delta / 3,0]$,

- $\phi_{3}^{+}=\phi_{3}^{-}$on $A_{r, 0}\left(\lambda^{\prime}, \lambda / 2\right)$.

Then we extend $\phi_{3}^{+}$to $T_{r}^{+}\left(\lambda^{\prime}, \epsilon^{\prime}\right)$ by using on $T_{r}^{+}(\lambda / 2, \epsilon / 2)$ the same parallel transport over $\zeta_{t}$ as in (19). Likewise, we extend $\phi_{3}^{-}$to $T_{r}^{-}\left(\lambda^{\prime}, \epsilon^{\prime}\right)$ by using on $T_{r}^{-}(\lambda / 2, \epsilon / 2)$ parallel transport over the reflection of $\zeta_{t}$ in the $x$-axis.

Because $\phi_{3}^{+}(p)=\phi_{3}^{-} \circ \chi(p)$ for $p \in T_{r}^{+}\left(\lambda^{\prime}\right)$, and $\phi_{3}^{+}, \phi_{3}^{-}$are $t$-invariant for $|t|$ small, they give rise to a Poisson morphism

$$
\phi_{3}^{+} \#_{\rho_{\partial \bar{D}(r)}} \phi_{3}^{-}: T_{r}^{+}\left(\lambda^{\prime}, \epsilon^{\prime}\right) \#_{\rho_{\partial \bar{D}(r)}} T_{r}^{-}\left(\lambda^{\prime}, \epsilon^{\prime}\right) \rightarrow H_{2,-r} .
$$

The equivalence of 2-calibrated foliations for all $r>0$ small enough is

$$
\phi= \begin{cases}\phi_{2} & \text { in } M^{L} \backslash\left(T_{r}^{+}\left(\lambda^{\prime}, \epsilon^{\prime}\right) \#_{\rho_{\partial \bar{D}(r)}} T_{r}^{-}\left(\lambda^{\prime}, \epsilon^{\prime}\right)\right), \\ \phi_{3}^{+} \#_{\rho_{\partial \bar{D}(r)}} \phi_{3}^{-} & \text {in } T_{r}^{+}\left(\lambda^{\prime}, \epsilon^{\prime}\right) \#_{\rho_{\partial \bar{D}(r)}} T_{r}^{-}\left(\lambda^{\prime}, \epsilon^{\prime}\right) .\end{cases}
$$

Let us now drop the assumption $\Omega_{2}=\Omega_{\tau}$. Let $\Omega_{u}$ be a path which is constant near its boundary and which connects $\Omega_{2}$ to $\Omega_{\mathbb{R}^{2 n+2}}$. 
Recall that the neighborhoods $T_{r}(\lambda, \epsilon)$ have been defined with respect to $\Omega_{2}$. By the parametric version of Lemma 18 (more specifically by the parametric version of the stable manifold theorem), we have smooth parametrizations $\Sigma_{u, r}, r \in\left(0, r^{\prime}\right]$. By compactness we can extend $\varphi_{r^{\prime}}$ to parametrizations

$$
\varphi_{u, r^{\prime}}:\left(T_{u, r^{\prime}}(\tilde{\lambda}), \Omega_{u}\right) \rightarrow\left(T(\tilde{\lambda}), d \alpha_{\text {can }}\right) .
$$

Then we define the subsets $T_{u, r}(\tilde{\lambda})$ by parallel transport of $T_{u, r^{\prime}}(\tilde{\lambda})$ over $x_{0}\left(r^{\prime}, r\right)$

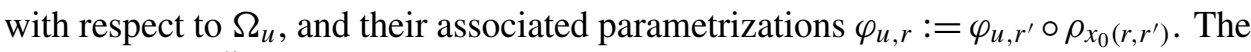
subsets $T_{u,-r}(\tilde{\lambda}) \backslash \Sigma_{u,-r}$ and their parametrizations are defined in the same manner.

We can assume without loss of generality that the inclusion

$$
T_{u, r}(\tilde{\lambda}) \subset T_{r}(\lambda / 2)
$$

holds for all $r \in\left(0, r^{\prime}\right]$. This is because the parametric version of the stable manifold theorem implies that

$$
\begin{aligned}
T(\lambda) \times\left[0, r^{\prime}\right] \times[0,1] & \rightarrow h_{0}, \\
(q, r, u) & \mapsto \rho_{u, x_{0}\left(r^{\prime}, 0\right)}\left(\varphi_{u, r}^{-1}(q)\right)
\end{aligned}
$$

is continuous, where by definition $\rho_{u, x_{0}(r, 0)}(p)=0 \in \mathbb{C}^{n+1}$ for $p \in \Sigma_{u, r}$.

We proceed to modify both $A_{r}^{+}\left(\lambda^{\prime}, \lambda / 2, \epsilon^{\prime}, \epsilon / 2\right)$ and $\phi_{3}$ in (19) just for values of $t$ in $[0, \delta]:$ let $b_{i}:[0, \delta] \rightarrow\left[2 \tilde{\lambda} / 3, \lambda^{\prime}\right]$ and $b_{o}:[0, \delta] \rightarrow[\tilde{\lambda} / 2, \lambda / 2]$ be monotone increasing functions which are constant on $[0,3 \delta / 4]$ and near $\delta$.

Let $v:[0, \delta] \rightarrow[0,1]$ be a orientation reversing smooth function which is constant on $[0, \delta / 2]$ and on $[3 \delta / 4, \delta]$.

We substitute $A_{r, t}\left(b_{o}(t), b_{i}(t)\right)$ for $A_{r, t}\left(\lambda^{\prime}, \lambda / 2\right)$ and

$$
\tilde{\phi}_{3, t}:=\rho_{0, y_{-r}(0, t)} \circ \varphi_{0,-r}^{-1} \circ \varphi_{v(t),-r} \circ \rho_{v(t), \partial \bar{D}_{\beta(t)}^{+}(r)} \circ \varphi_{v(t), r}^{-1} \circ \varphi_{0, r} \circ \rho_{0, y_{r}(t, 0)}
$$

defined on $A_{r, t}\left(b_{o}(t), b_{i}(t)\right)$ for $\phi_{3, t}$ in (19). Note that the modification of the symplectic form only occurs when the domain has been modified to $A_{r, t}(2 \tilde{\lambda} / 3, \tilde{\lambda} / 2)$.

By the inclusion in (22), the image of $A_{r, t}(2 \tilde{\lambda} / 3, \tilde{\lambda} / 2)$ under $\varphi_{v(t), r}^{-1} \circ \varphi_{0, r} \circ \rho_{0, y_{r}(t, 0)}$ is contained in $T_{r}(\lambda / 2)$. If in addition $r>0$ is small enough, control on the $C^{0}$-norm of $\rho_{v(t), \partial \bar{D}_{\beta(t)}^{+}(r)}$ by $r$ implies that

$$
\rho_{v(t), \partial \bar{D}_{\beta(t)}^{+}(r)} \circ \varphi_{v(t), r}^{-1} \circ \varphi_{0, r} \circ \rho_{0, y_{r}(t, 0)}
$$

sends $A_{r, t}(2 \tilde{\lambda} / 3, \tilde{\lambda} / 2)$ into $T_{u(t),-r}(\tilde{\lambda}) \backslash \Sigma_{u(t),-r}$, so we can compose with the chart $\varphi_{v(t),-r}$. Therefore $\tilde{\phi}_{3, t}$ is well defined and for $t \in[0, \delta / 2]$ we are in the situation $\Omega_{2}=\Omega_{\mathbb{R}^{2 n+2}}$.

Then we have to choose $\Omega_{\tau}$ whose conjugation by $\varphi_{0, r}\left(\Omega_{0}=\Omega_{\mathbb{R}^{2 n+2}}\right)$ is a Dehn twist supported in the interior of $T(\tilde{\lambda} / 2)$. 
We can use the same pattern to modify the isotopy needed to apply the extension lemma with parameters (this time the radii of the annuli vary with $t$ ). The result is an extension $\phi_{3}^{+}$which is $t$-invariant for $t \in[0, \delta / 4]$, and for which on $A_{r, 0}(2 \tilde{\lambda} / 3, \tilde{\lambda} / 2)$ the chart $\varphi_{0, t}$ conjugates to a Dehn twist supported on $T(\tilde{\lambda} / 2)$.

As we did in the previous stage, we construct the extension $\phi_{3}^{-}$using as domain and curves the reflection of the previous data in the $x$-axis. Then $\phi$, defined as in (21), is the equivalence of 2-calibrated foliations which proves the theorem.

Remark 28. Similarly, for $n>1$ and every $r>0$ small enough one constructs equivalences

$$
\begin{aligned}
\left(-M^{L^{-}}, \mathscr{F}^{L^{-}}, \omega^{L^{-}}\right) & \rightarrow\left(M^{-\mu_{L}}, \mathscr{F}^{-\mu_{L}}, \omega^{-\mu_{L}}\right), \\
\left(M^{-L}, \mathscr{F}^{-L}, \omega^{-L}\right) & \rightarrow\left(M^{\left.\mu_{L^{-}}, \mathscr{F}_{F} \mu_{L^{-}}, \omega^{\mu_{L^{-}}}\right) .}\right.
\end{aligned}
$$

\section{Lefschetz pencil structures and transverse taut foliations}

Let $\left(M^{2 n+1}, \mathscr{F}, \omega\right)$ be an integral 2-calibrated foliation. In this section we gather information on the intersection of a Donaldson-type submanifold with the leaves of $\mathscr{F}$ using Lefschetz pencil structures. We also describe the relation between two Donaldson type submanifolds belonging to the same Lefschetz pencil.

We start by saying a few words about how Donaldson-type submanifolds $W$ are constructed, and how the failure of standard Morse theoretic methods to describe the topology of $W \cap F, F \in \mathscr{F}$, leads to the use of Lefschetz pencil structures to address this problem.

Let us fix $J$ a leafwise almost complex structure compatible with $\omega$. If $J$ is integrable then by definition $(M, \mathscr{F}, J)$ is a Levi-flat manifold, and the line bundle $L_{\omega}$ whose curvature is $-2 \pi i \omega$ is a positive CR line bundle. According to [Ohsawa and Sibony 2000], large powers of $L_{\omega}$ (suitably twisted) have plenty of CR sections. In particular there exist CR sections leafwise transverse to the zero section of $L_{\omega}^{\otimes k}$. The zero set of any such section is a codimension-two CR submanifold, or a divisor, intersecting $\mathscr{F}$ transversely.

In general $J$ is not integrable. However $L_{\omega}^{\otimes k} \otimes \mathbb{C}^{l}$ has sections $s$ which are both close to being $J$-holomorphic in an appropriate sense and leafwise transverse to the zero section of $L_{\omega}^{\otimes k} \otimes \mathbb{C}^{l}$ [Ibort and Martínez Torres 2004a, Corollary 1.2]. As a consequence $W=s^{-1}(0)$ is a 2 -calibrated submanifold of $(M, \mathscr{F}, \omega)$ of codimension $2 l$, and it is what we call a Donaldson-type submanifold. The topology of $W$ and the topology of $M$ are related by a Lefschetz hyperplane-type result: the section $s$ is chosen so that $\log s \bar{s}$ is a Morse function. By approximate $J$-holomorphicity the index of critical points is greater than $n-l$, from which the vanishing of $\pi_{i}(M, W)$, $0 \leq i \leq n-l-1$, follows [ibid., Corollary 1.2]. In particular the common zero set 
of $n-1$ well-chosen such sections of $L_{\omega}^{\otimes k}$ is $W^{3} \hookrightarrow M$, a connected Donaldson type 3-dimensional submanifold.

For any given leaf $F$, it is tempting to study the topology of $W^{3} \cap F$ by the same Morse-theoretic methods. It is always possible to arrange the tuple $s=\left(s_{1}, \ldots, s_{n-1}\right)$ so that the restriction of $\log s \bar{s}^{2}$ to $F$ is a Morse function. The usual Morse theoretic argument [Donaldson 1996; Auroux 1997, Proposition 2] implies that critical points have index greater than one, and therefore if $F$ is compact (and hence $W^{3} \cap F$ is compact), then $W^{3} \cap F$ is connected. If $F$ is not compact then the restriction of $\log s \bar{s}^{2}$ to $W^{3} \cap F$ is never proper, and it is not clear how the information on index of critical points can be translated into topological information about $W^{3} \cap F$.

A second approach to studying the topology of complex manifolds is via holomorphic Morse functions and Picard-Lefschetz theory. In our setting these are Lefschetz pencil decompositions of $(M, \mathscr{F})$ provided by ratios of suitable pairs of sections $s_{1}, s_{2}$ of $L_{\omega}^{\otimes k}$. Very much as we did in the previous section with the complex quadratic function $h$, we are going to use the parallel transport associated to a Lefschetz pencil decomposition to "reconstruct" a leaf $F$ from its intersection with a regular fiber of the pencil (the previous section contains the analysis around a critical point of the holomorphic Morse function). This will be enough to prove Theorem 2 . Parallel transport is also the way to compare two regular fibers of a given Lefschetz pencil structure, showing that they differ by a sequence of generalized Dehn twists.

5.1. Lefschetz pencil structures. We recall the notion of Lefschetz pencil structure and the main existence result, and collect some necessary results regarding the associated leafwise parallel transport.

Definition 29. Let $x \in(M, \mathscr{F}, \omega)$. A chart $\varphi_{x}:\left(\mathbb{C}^{n} \times \mathbb{R}, 0\right) \rightarrow(M, x)$ is compatible with $(\mathscr{F}, \omega)$ if it is a foliated chart, and $\varphi_{x}^{*} \omega$ restricted to the leaf through the origin is of type $(1,1)$ at the origin.

Definition 30 [Ibort and Martínez Torres 2004b]. A Lefschetz pencil structure for $(M, \mathscr{F}, \omega)$ is given by a triple $(f, B, \Delta)$, where $B \subset M$ is a codimension-four 2-calibrated submanifold and $f: M \backslash B \rightarrow \mathbb{C P}^{1}$ is a smooth map such that:

(i) $f$ is a leafwise submersion away from $\Delta$, a 1-dimensional manifold transverse to $\mathscr{F}_{F}$ where the restriction of the differential of $f$ to $\mathscr{F}$ vanishes. The fibers of the restriction of $f$ to $M \backslash(B \cup \Delta)$ are 2-calibrated submanifolds.

(ii) Around any critical point $c \in \Delta$ there exist Morse coordinates $z_{1}, \ldots, z_{n}, t$ compatible with $(\mathscr{F}, \omega)$, and a standard complex affine coordinate on $\mathbb{C P}^{1}$ such that

$$
f(z, t)=z_{1}^{2}+\cdots+z_{n}^{2}+\sigma(t),
$$


where $\sigma \in C^{\infty}(\mathbb{R}, \mathbb{C})$.

(iii) Around any base point $b \in B$ there exist coordinates $z_{1}, \ldots, z_{n}, t$ compatible with $(\mathscr{F}, \omega)$, and a standard complex affine coordinate on $\mathbb{C P}^{1}$ such that $B \equiv z_{1}=z_{2}=0$ and $f(z, t)=z_{1} / z_{2}$.

(iv) $f(\Delta)$ is an immersed curve in general position.

For each regular value $z \in \mathbb{C P}^{1} \backslash f(\Delta)$, the regular fiber is the compactification $W_{z}:=f^{-1}(z) \cup B$, which is a (compact) 2-calibrated submanifold.

Theorem 31 [Ibort and Martínez Torres 2004b, Theorem 1.2]. Let $(M, \mathscr{F}, \omega)$ be an integral 2-calibrated foliation and let e be an integral lift of $[\omega]$. Then for all $k \gg 1$ there exist Lefschetz pencils $\left(f_{k}, B_{k}, \Delta_{k}\right)$ such that:

(i) The regular fibers are Poincaré dual to ke.

(ii) The inclusion $l_{k}: W_{k} \hookrightarrow M$ induces maps

$$
l_{k *}: \pi_{i}\left(W_{k}\right) \rightarrow \pi_{i}(M) \text { and } l_{k *}: H_{i}\left(W_{k} ; \mathbb{Z}\right) \rightarrow H_{i}(M ; \mathbb{Z}),
$$

which are isomorphisms for $i \leq n-2$ and epimorphisms for $i=n-1$.

5.1.1. Leafwise symplectic parallel transport. Let $(f, B, \Delta)$ be a Lefschetz pencil structure for $(M, \mathscr{F}, \omega)$. Away from the union of base points and critical points $B \cup \Delta$, the fibers of $f$ are 2-calibrated submanifolds. In particular for any point $p \notin B \cup \Delta$ this is equivalent to the tangent space of the leaf through $p$ and the tangent space to the fiber of $f$ through $p$ intersecting transversely in a symplectic subspace. Therefore the leafwise symplectic orthogonals to the fibers define an Ehresmann connection for $f$, which we denote by $\mathscr{H}$ and also refer to as the horizontal distribution.

The Ehresmann connection $\mathscr{H}$ is defined in the noncompact manifold $M \backslash(B \cup \Delta)$. We are going to show that we have good control on parallel transport near base points and critical points.

Let $F$ be a leaf of the foliation. Let $B_{F}$ denote the codimension-four submanifold of base points in $F$ and let $\Delta_{F}$ denote the dimension zero submanifold of critical points in $F$. The image $f\left(\Delta_{F}\right)$ is a possibly countable collection of points in the immersed curve $f(\Delta)$. In particular it is easy to construct curves $\gamma \subset \mathbb{C P}^{1}$ which do not intersect $f\left(\Delta_{F}\right)$ (or to homotope curves to avoid $f\left(\Delta_{F}\right)$ ).

Lemma 32. Let $\gamma \subset \mathbb{C P}^{1}$ be a curve not intersecting $f\left(\Delta_{F}\right)$. Then parallel transport $\rho_{\gamma}: f_{\gamma(0)}^{-1} \cap F \rightarrow f_{\gamma(1)}^{-1} \cap F$ is a well defined symplectomorphism. Moreover, it extends smoothly to a symplectomorphism $\rho_{\gamma}: W_{\gamma(0)} \cap F \rightarrow W_{\gamma(1)} \cap F$ which is the identity on $B_{F}$. In particular if $\gamma$ misses $f(\Delta)$, it induces an equivalence of 2-calibrated foliations $\rho_{\gamma}: W_{\gamma(0)} \rightarrow W_{\gamma(1)}$ which is the identity on $B$. 
Proof. A standard procedure in this situation is to blow up $B$ along its leafwise almost complex normal directions.

We consider the following model for the blow up as a submanifold of $M \times \mathbb{C P}^{1}$ : we let $\tilde{M}$ be the union of the graph of $f$ and $B \times \mathbb{C P}^{1}$. We need to show that $\tilde{M}$ is a submanifold around points in $B \times \mathbb{C P}^{1}$.

Around a point $b \in B$, Theorem 31 provides coordinates $z_{1}, \ldots, z_{n}, t$ and a standard affine coordinate on $\mathbb{C P}^{1}$ such that $B \cong z_{1}=z_{2}=0$ and $f=z_{1} / z_{2}$. This is equivalent to saying that near $b$ the graph of $f$ is given by

$$
\left(\left(z_{1}, \ldots, z_{n}, t\right),\left[z_{1}: z_{2}\right]\right) \subset M \times \mathbb{C P}^{1} .
$$

In these coordinates $\tilde{M}$ coincides with the complex blow up in the first two coordinates, and therefore it is a submanifold.

The first projection restricts to the blow down map $\pi: \tilde{M} \rightarrow M$, which is the identity away from $B$ and collapses each $\{b\} \times \mathbb{C P}^{1} \subset \tilde{M}$ to $b \in B \subset M$. The restriction to $\tilde{M}$ of the second projection on $M \times \mathbb{C} \mathbb{P}^{1}$ defines an extension of $f$, $\tilde{f}: \tilde{M} \rightarrow \mathbb{C P}^{1}$. Because we are blowing up directions inside leaves we have an induced foliation $\tilde{\mathscr{F}}$, and the blow down map is a map of foliated manifolds.

The fibers of $\tilde{f}$ are transverse to $\tilde{\mathscr{F}}$, and by construction the restriction of the projection $\pi: \tilde{f}_{z} \rightarrow W_{z}$ is a diffeomorphism of foliated manifolds. We let $\tilde{F}$ denote the leaf mapping into $F$.

Let $\tilde{\omega}$ denote the pullback of $\omega$ by the blow down map. We claim that the intersection of the fibers of $\tilde{f}$ with $\tilde{\mathscr{F}}$ are symplectic manifolds with respect to $\tilde{w}_{\tilde{F}}$, and therefore there is an associated leafwise Ehresmann connection which extends $\mathscr{H}$. At a point $p=\left(b,\left[z_{1}: z_{2}\right]\right)$, say $z_{2} \neq 0$, the tangent space $T_{\left[z_{1}: z_{2}\right]} \mathbb{C} \mathbb{P}^{1} \subset T_{\left(b,\left[z_{1}: z_{2}\right]\right)} \tilde{F}$ is in the kernel of $\tilde{\omega}_{\mathscr{F}}$ because the blow down map collapses the $\mathbb{C P}^{1}$ factor into the point $b$. The subspace $T_{\left(b,\left[z_{1}: z_{2}\right]\right)} \tilde{f} \cap T_{\left(b,\left[z_{1}: z_{2}\right]\right)} \tilde{F}$ is complementary to $T_{\left[z_{1}: z_{2}\right]} \mathbb{C P} \mathbb{P}^{1}$ and it is mapped isomorphically into $T_{b} f_{z_{1} / z_{2}} \cap T_{b} F$, and the latter is symplectic with respect to $\omega_{\mathscr{F}}$ (alternatively, in local coordinates about the base point $T_{b} f_{z_{1} / z_{2}} \cap T_{b} F$ is a complex hyperplane of $T_{0} \mathbb{C}^{n}$, and therefore it is symplectic with respect to $\omega_{\mathscr{F}}$ because the symplectic form has type $(1,1)$ at the origin). Thus, the blow down map identifies $\tilde{f}_{z}$ and $W_{z}$ as 2-calibrated foliations.

Once we have described the kernel of $\tilde{w}_{\tilde{F}}$, it is easy to see that the horizontal lift of $\gamma \subset \mathbb{C P}^{1}$ starting at $(b, \gamma(0))$ is exactly $(b, \gamma)$.

It is clear that parallel transport defines a Poisson equivalence. It is obviously an equivalence of 2-calibrated foliations because (the induced) coorientations are preserved, and the 2-calibrations are restriction of the same closed 2-form on $M$.

Let $c \in \Delta$ be a critical point and let us apply Theorem 31 to construct Morse coordinates for $f$ centered at $c$. By restricting Morse coordinates to the leaf $F$ containing $c$, we obtain Morse coordinates for the restriction of $f$ to $F$. Since the restriction of $\omega_{\mathscr{F}}$ to $F$ is mapped to a symplectic form of type $(1,1)$ at the origin, 
leafwise parallel transport near $c$ corresponds to parallel transport in $\mathbb{C}^{n} \backslash\{0\}$ near 0 for the function $h$ with respect to a symplectic form of type $(1,1)$ at the origin.

Let us consider the following system of neighborhoods of the critical point $0 \in \mathbb{C}^{n}$ [Seidel 2003, Section 1.2]: we fix the standard symplectic form $\Omega_{\mathbb{R}^{2 n}}$ and define $\Sigma_{z}$, $z \in \mathbb{C}$, to be the Lagrangian sphere of points in $h_{z}$ whose parallel transport over the radial segment converges to the origin. For some $r_{0}>0$ we fix the parametrization

$$
\varphi_{r_{0}}:\left(T_{r_{0}}(\lambda), \Omega_{\mathbb{R}^{2 n}}\right) \rightarrow\left(T(\lambda), d \alpha_{\text {can }}\right) .
$$

For any $z \in \mathbb{C}$ small enough we define $T_{z}(\lambda) \backslash \Sigma_{z}$ by radial parallel transport to the origin and then to $r_{0}$. Of course, $T_{z}(\lambda)$ denotes the union of $T_{z}(\lambda) \backslash \Sigma_{z}$ and $\Sigma_{z}$.

Then

$$
\mathscr{T}(\lambda, r)=\bigcup_{z \in \bar{D}(r)} T_{z}(\lambda), \quad \lambda, r>0,
$$

is a system of neighborhoods of the origin. We also have the corresponding annular subsets $\mathscr{A}\left(\lambda, \lambda^{\prime}, r, r^{\prime}\right)$.

Lemma 33. Let $\Omega_{u}, u \in K$, be a compact family of symplectic forms defined on a neighborhood $W$ of $0 \in \mathbb{C}^{n}$ which make the fibers $h_{z}$ symplectic submanifolds. Let us fix any $\lambda, r>0, \lambda^{\prime} \in(0, \lambda)$ and $r^{\prime} \in(0, r)$. Then there exists $\delta>0$ such that for any curve $\gamma \subset \bar{D}\left(r^{\prime}\right) \backslash\{0\}$ having the $C^{1}$-norm of $\gamma-\gamma(0)$ bounded by $\delta$, the horizontal lift $\tilde{\gamma}_{u}$ starting at any $p \in \mathscr{T}\left(\lambda^{\prime}, r^{\prime}\right)$ is contained in $\mathscr{T}(\lambda, r)$, for all $u \in K$.

Proof. Let $\mathscr{C}$ denote the topological space of (piecewise embedded or constant) curves contained in $\bar{D}(r)$ relative to the $C^{1}$-topology. Let us consider the subset

$$
E=\left\{(\gamma, p, u, v) \subset \mathscr{C} \times \mathscr{A}\left(\lambda^{\prime}, \tilde{\lambda}, r, r^{\prime}\right) \times K \times[0,1] \mid \gamma(0)=h(p)\right\},
$$

and let us define the continuous map

$$
\begin{aligned}
G: E & \rightarrow W, \\
(\gamma, p, u, v) & \mapsto \tilde{\gamma}_{u}(v),
\end{aligned}
$$

by sending a tuple to the evaluation for time $v$ of the horizontal lift of $\gamma$ with respect to $\Omega_{u}$ starting at $p$. The map is not everywhere defined since horizontal lifts may leave $W$ or converge to the critical point, which is exactly what we want to control. However, inside $E$ we have the subset $\mathscr{A}\left(\lambda^{\prime}, \tilde{\lambda}, r, r^{\prime}\right) \times K \times[0,1]$ corresponding to constant curves. The restriction of $G$ to this subset is the first projection. By continuity, an open neighborhood of $\mathscr{A}\left(\lambda^{\prime}, \tilde{\lambda}, r, r^{\prime}\right)$ inside $E$ is sent into $\mathscr{T}(\lambda, r)$. Because on $E$ we have the topology induced by the product topology, we conclude the existence of $\delta$ such that curves with $\|\gamma-\gamma(0)\|_{C^{1}}<\delta, \gamma(0) \in \bar{D}\left(r^{\prime}\right)$, have horizontal lift starting at points in $A_{\gamma(0)}\left(\lambda^{\prime}, \tilde{\lambda}\right)$ contained in $\mathscr{T}(\lambda, r)$. If in addition such a small curve does not contain $0 \in \mathbb{C}$, the connectivity argument already used 
a couple of times implies that horizontal lifts starting at points in $T_{\gamma(0)}\left(\lambda^{\prime}\right)$ remain inside $\mathscr{T}(\lambda, r)$, and this proves the lemma.

Remark 34. Let $\sigma \in \mathbb{C}$ and consider the constant perturbation of the complex quadratic form $h+\sigma$. Note that in the definition of $\mathscr{T}(\lambda, r) \subset \mathbb{C}^{n}$ for $h$ given in (25), if we replace radial segments joining a point $z$ to the origin by segments joining $z$ to $\sigma$, we get exactly the same subset $\mathscr{T}(\lambda, r)$. Now assume that the parameter $u \in K$ in Lemma 33 describes not just the variation of symplectic forms, but a perturbation of $h$ by a constant $\sigma(u)$. Then Lemma 33 holds replacing in the statement $h$ by $h+\sigma$ and the disk of radius $r^{\prime}$ by the disk of radius $r^{\prime}$ centered at $\sigma(u)$.

\subsection{Connected components of $W_{z} \cap F$ and leafwise parallel transport. Let us} fix $z_{0} \in \mathbb{C P}^{1}$ a regular value for $f$. Let $\gamma \subset \mathbb{C P}^{1}$ be a loop based at $z_{0}$ with empty intersection with $f\left(\Delta_{F}\right)$. Lemma 32 implies that parallel transport over $\gamma$ defines a diffeomorphism on $W_{z_{0}} \cap F$ (actually a symplectomorphism). Therefore the loop acts on connected components of $W_{z_{0}} \cap F$ and the action descends to $\pi_{1}\left(\mathbb{C P}^{1} \backslash f\left(\Delta_{F}\right), z_{0}\right)$.

Proposition 35. The action of $\pi_{1}\left(\mathbb{C P}^{1} \backslash f\left(\Delta_{F}\right), z_{0}\right)$ on connected components of $W_{z_{0}} \cap F$ is trivial.

Proof. Let $\gamma$ be a loop based at $z_{0}$ and not intersecting $f\left(\Delta_{F}\right)$. Consider $H_{S}$ a homotopy connecting $H_{0}=\gamma$ with the constant path $z_{0}$. We can assume without loss of generality that $H$ misses a point of $\mathbb{C P}^{1}$, and therefore compose with an affine coordinate chart and work in $\mathbb{C}$.

We can assume as well that the curves $\gamma_{s}$ in the homotopy coincide in the complement of an interval $[a, b] \subset[0,1]$, and the $C^{1}$-norm of $\gamma_{\mid[a, b]}-\gamma(a)$ (rescaled to have domain $[0,1])$ is bounded by any given $\delta>0$ : by breaking the domain of $H$ into $n^{2}$ squares of side $1 / n$, we can write $H$ as composition of $n^{2}$ homotopies with the above property. It is possible that the starting curve $\gamma_{0}$ of each of the $n^{2}$ homotopies does intersect $f\left(\Delta_{F}\right)$, but intersections can be removed after a perturbation with does not affect the behavior we demand on the curves $\gamma_{s}$.

We are going to control how the lifts of the curves in the homotopy behave near $\Delta_{F}$ using Morse coordinates, and away from $\Delta_{F}$ using a compactness argument.

Let $c \in \Delta$ and let us construct Morse coordinates $z_{1}, \ldots, z_{n}, t$ as in Definition 30 . We say that the restriction of the coordinates to each plaque in their domain are Morse coordinates for the restriction of $f$ to the plaque. In Morse coordinates for a given plaque the restriction of $f$ transforms into $h+\sigma(t)$ and $\omega_{\mathscr{F}}$ transforms into a symplectic form of making the fibers $(h+\sigma(t))_{z}$ symplectic manifolds (this is because we can construct Morse coordinates centered at any point in $\Delta$, and in Morse coordinates on the plaque containing $c$ the perturbation $\sigma(t)$ can be taken to be trivial and $\omega_{\mathscr{F}}$ becomes a symplectic form of type $(1,1)$ at the origin). 
Let us cover $\Delta$ with a finite number of Morse coordinates and let us consider their associated 1-parameter families of Morse coordinates on their plaques. Let us take $\lambda, r>0$ such that $\mathscr{T}(\lambda, r)$ as defined in (25) is contained in the image of Morse coordinates for each of the plaques. Let us also pick $\lambda^{\prime} \in(0, \lambda)$ and $r^{\prime} \in(0, r)$, and denote by $U$ the points in $\tilde{M}$ whose image under at least one of the sets of Morse coordinates on its plaque is contained in $\mathcal{T}\left(\lambda^{\prime}, r^{\prime}\right)$. Note that $U$ is a neighborhood of $\Delta$.

For each of our Morse coordinates, its 1-parameter family of Morse coordinates on plaques fulfills the hypothesis of Lemma 33, or rather Remark 34 (we assume that the parameter space is a compact interval, and that these compact intervals cover $\Delta$ ). Let $\delta_{1}$ be a $C^{1}$-bound provided by Remark 34 and valid for the finite number of 1-parameter families.

Let $V \subset V^{\prime}$ be open neighborhoods of $\Delta$ in $\tilde{M}$ such that $V \subset \bar{V} \subset V^{\prime} \subset U$. Because $\tilde{M} \backslash V^{\prime}$ is compact, there exists $\delta_{2}>0$ such that for any $p \in \tilde{M} \backslash V^{\prime}$ and any curve $\gamma \subset \mathbb{C P} \mathbb{P}^{1}$ starting at $\tilde{f}(p)$ and such that the $C^{1}$-norm of $\gamma-\gamma(0)$ is bounded by $\delta_{2}$, the horizontal lift $\tilde{\gamma}$ starting at $p$ is contained in $\tilde{M} \backslash V$.

Let $\delta$ be the minimum of $\delta_{1}$ and $\delta_{2}$, and let us assume that for each $\gamma_{s}$ in our homotopy $H$ the $C^{1}$-norm of $\gamma_{s \mid[a, b]}-\gamma_{s}(a)$ is smaller than $\delta$. Let $\gamma_{0}$ and $\gamma_{1}$ be the starting and ending curve of the homotopy and let $\tilde{\gamma}_{0}$ and $\tilde{\gamma}_{1}$ be their respective horizontal lifts starting at $p \in W_{z_{0}} \cap F$. We claim that $\tilde{\gamma}_{0}(1)$ and $\tilde{\gamma}_{1}(1)$ can be connected by a path in $W_{z_{0}} \cap F$, which suffices to prove the proposition.

Recall that $\gamma_{s}, s \in[0,1]$, is independent of $s$ in the complement of $[a, b] \subset[0,1]$.

Let us suppose that $\tilde{\gamma}_{0}(a) \in \tilde{M} \backslash V^{\prime}$. Because of the $C^{1}$-bound on $\gamma_{s \mid[a, b]}-\gamma_{s}(a)$, $s \in[0,1]$, the horizontal lifts of $\gamma_{s \mid[a, b]}$ starting at $\tilde{\gamma}_{0}(a)$ are defined for all $s \in[a, b]$ and belong to $\tilde{M} \backslash V$. In particular $\tilde{\gamma}_{s}(b)$ is a curve in the fiber $\tilde{f}_{\gamma_{0}(b)}$. Since the curves $\gamma_{s \mid[b, 1]}$ are all equal and avoid $f\left(\Delta_{F}\right)$, we can construct the horizontal lift starting at all points in the path $\tilde{\gamma}_{s}(b)$. What we just proved is that the homotopy $H_{s}$ has a well-defined lift starting at $p$, and therefore $\tilde{\gamma}_{s}(1)$ connects $\tilde{\gamma}_{0}(1)$ to $\tilde{\gamma}_{1}(1)$.

If $\tilde{\gamma}_{0}(a) \in V$ then it also belongs to $U$. If we compose with one of the fixed Morse coordinates on the plaque $u_{0}$ containing $\tilde{\gamma}_{0}(a)$, the point $\tilde{\gamma}_{0}(a)$ is sent to $q \in \mathscr{T}\left(\lambda^{\prime}, r^{\prime}\right)$. The curves $\gamma_{0 \mid[a, b]}$ and $\gamma_{1_{\mid[a, b]}}$ meet the hypothesis of Lemma 33 (Remark 34), and therefore their horizontal lifts starting at $q$ are contained in $\mathscr{T}(\lambda, r)$. In particular the images $q_{0}$ and $q_{1}$ of $\tilde{\gamma}_{0}(b)$ and $\tilde{\gamma}_{1}(b)$, respectively, belong to $\left(h+\sigma\left(u_{0}\right)\right)_{\gamma_{0}(b)}$. All regular fibers of $h+\sigma\left(u_{0}\right)$ in $\mathscr{T}(\lambda, r)$ are diffeomorphic to $T(\lambda)$ and therefore they are connected. Let $\zeta$ be a path in $\left(h+\sigma\left(u_{0}\right)\right)_{\gamma_{0}(b)}$ connecting $q_{0}$ to $q_{1}$. Let us also denote by $\zeta$ its image in the plaque $u_{0}$ by the Morse chart, which belongs to $\tilde{f}_{\gamma_{0}(b)}$. Then the ending points of the lifts of $\gamma_{0 \mid[b, 1]}$ starting at $\zeta(v), v \in[0,1]$, connect $\tilde{\gamma}_{0}(1)$ to $\tilde{\gamma}_{1}(1)$.

Proposition 35 is the key result to "spread" a connected component of $W_{z_{0}} \cap F$ onto $F$. Before, we need to show that $W_{z_{0}} \cap F$ is always nonempty. For that it 
suffices to prove that $\tilde{f}(F)$ contains some regular value $z$ of $\tilde{f}$, because in that case we can use parallel transport over a curve joining $z$ to $z_{0}$ and avoiding singular values of $f\left(\Delta_{F}\right)$ to find points in $W_{z_{0}} \cap F$ : because $\tilde{M}$ is compact the regular values of $\tilde{f}$ (which are the regular values of $f$ ) are an open dense subset. The subset $\tilde{f}(F) \subset \mathbb{C P}^{1}$ has not empty interior and therefore it contains regular values.

Theorem 36. Let $\left(M^{2 n+1}, \mathscr{F}, \omega\right), n>1$, be a 2-calibrated foliation and $(f, B, \Delta)$ be a Lefschetz pencil structure as in Definition 30. Then any regular fiber $W$ of the pencil intersects every leaf of $\mathscr{F}$ in a unique connected component.

Proof. Let $z_{0}$ be a regular value and let $F$ be a leaf. We let $C$ be a nonempty connected component of $W_{z_{0}} \cap F$ (it always exists since $W_{z_{0}} \cap F$ is nonempty). Let us define $\Gamma_{C}$ to be the set of horizontal curves starting at $C$ and whose projection $\tilde{f} \circ \zeta$ is either an embedded curve or constant. We define

$$
F_{C}:=\left\{p \in F \backslash \Delta_{F} \mid \text { there exists } \zeta \in \Gamma_{C}, \zeta(1)=p\right\} .
$$

By construction $F_{C}$ is nonempty, connected and contains $C$. We want to show that it is open.

Let $p \in F_{C}$ such that the horizontal curve $\zeta$ connects $x \in C$ with $p$. Let us suppose that the curve $\tilde{f} \circ \zeta$ is embedded (it is not constant). Then we can find a 1parameter family of embedded curves $\gamma_{s}, s \in(-\epsilon, \epsilon)$, defined for time $v \in[0,1+\epsilon]$, and such that the restriction of $\gamma_{0}$ to $[0,1]$ is $\tilde{f} \circ \zeta$. Because $\zeta$ is contained in $\tilde{M} \backslash \Delta$, a compactness argument implies that there exists $A$ an open neighborhood of $x$ inside $C$ and $\epsilon^{\prime}>0$, such that the horizontal lift of $\gamma_{s \mid\left[0,1+\epsilon^{\prime}\right]}$ starting at any point in $A$ exists for all $s \in\left(-\epsilon^{\prime}, \epsilon^{\prime}\right)$. It is clear that for $\epsilon^{\prime}$ small enough

$$
U_{p}=\left\{y \in F \mid y=\tilde{\gamma}_{s}(v), \tilde{\gamma}_{s}(0) \in A, v \in\left(1-\epsilon^{\prime}, 1+\epsilon^{\prime}\right), s \in\left(-\epsilon^{\prime}, \epsilon^{\prime}\right)\right\}
$$

is a neighborhood of $p$ in $F_{C}$.

If $\zeta$ is constant we make the previous construction for a family of radial curves starting at $z_{0}$, and the open neighborhood is obtained considering horizontal lifts for time $v \in\left[0, \epsilon^{\prime}\right.$ ) starting at a neighborhood $A$ of $p$ inside $C$ (we would be "spreading" the open subset $A$ ).

We claim that $F_{C}$ does not contain a connected component of $W_{z_{0}} \cap F$ different from $C$. Suppose the contrary. Then we would have a loop $\gamma$ with a horizontal lift connecting two different connected components of $W_{z_{0}} \cap B$. Since after a small perturbation we can assume without loss of generality that $\gamma$ does not intersect $\Delta_{F}$, this would contradict Proposition 35.

Because it is clear that any point in $F \backslash \Delta_{F}$ can be connected to $W_{z_{0}} \cap F$ by a horizontal curve lifting an embedded curve, we conclude that connected components of $W_{z_{0}} \cap F$ are in bijection with connected components of $F$, and this proves that $W_{z_{0}} \cap F$ is connected. 
Proof of Theorem 2. Let $(M, \mathscr{F}, \omega)$ be a 2-calibrated foliation. If it is not integral, compactness of $M$ implies that we can slightly modify $\omega$ into $\omega^{\prime}$ so that a suitable multiple $k \omega^{\prime}$ defines an integral homology class. Theorem 31 implies the existence of a Lefschetz pencil $(f, B, \Delta)$.

Therefore by Theorem 36 any regular fiber $\left(W, \mathscr{F}_{W}, k \omega_{W}^{\prime}\right)$ intersects every leaf in a connected component. If the dimension of $W$ is bigger than 3 , we apply the same construction to $\left(W, \mathscr{F}_{W}, k \omega_{W}^{\prime}\right)$. By induction we end up with a 3-dimensional manifold with a taut foliation $\left(W^{3}, \mathscr{F}_{W}\right) \hookrightarrow\left(M, \mathscr{F}_{F}, \omega\right)$, whose intersection with every leaf of $\mathscr{F}$ is connected.

Proof of Theorem 4. Let $l: W \hookrightarrow M$ be a submanifold as in Theorem 2. Because for all $F \in \mathscr{F}$ the intersection $W \cap F$ is connected, the map $l$ descends to a bijection of leaf spaces

$$
\tilde{l}: W / \mathscr{F}_{W} \rightarrow M / \mathscr{F}
$$

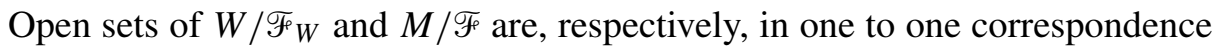
with saturated open sets of $W$ and $M$.

Let $V$ be an saturated open set of $(M, \mathscr{F})$. By definition $W \cap V$ is an open set of $W$ which is clearly saturated (even without the assumption of $\tilde{l}$ being a bijection) and this shows that $\tilde{l}$ is continuous.

Now let $V$ be an open saturated set of $\left(W, \mathscr{F}_{W}\right)$. We want to show that its saturation in $(M, \mathscr{F})$, denoted by $\bar{V}^{\mathscr{F}}$, is open, to conclude that $\tilde{l}$ is open.

If $V$ is a saturated set and $x \in V$, then $x$ is an interior point if and only if for some $T_{x}$ a local manifold through $x$ transverse to the foliation, $x$ is an interior point of $T_{x} \cap V$. Hence, every $x \in V$ is an interior point of $\bar{V}^{\mathscr{F}}$. By using the holonomy, if a point in a leaf is interior, the whole leaf is made of interior points. Since every leaf of $\bar{V}^{\mathscr{F}}$ intersects $V, \bar{V}^{\mathscr{F}}$ is open, and this proves the theorem.

5.3. Regular fibers and Lagrangian surgery. Let $W$ be a regular fiber of a Lefschetz pencil structure for $(M, \mathscr{F}, \omega)$. Theorems 36 and 31 describe the topology of $W / \mathscr{F}_{W}$ and part of the homology and homotopy of $W$ in terms of the corresponding data for $(M, \mathscr{F})$. We want to understand how different regular fibers of the pencil are related as 2-calibrated foliations.

Let $z$ and $z^{\prime}$ be regular values of the pencil belonging to the same connected component of $\mathbb{C} \mathbb{P}^{1} \backslash f(\Delta)$, and let $\gamma$ be a curve in that connected component connecting $z$ to $z^{\prime}$. Then Lemma 32 implies that $\rho_{\gamma}: W_{z} \rightarrow W_{z^{\prime}}$ is an equivalence of 2-calibrated foliations.

We notice that any two arbitrary regular values $z$ and $z^{\prime}$ can always be joined by a curve $\gamma$ transverse to $f(\Delta)$.

Theorem 37. Let $z, z^{\prime} \in \mathbb{C P}^{1}$ be two regular values. Let $\gamma$ be an embedded curve joining $z$ and $z^{\prime}$ and transverse to $f(\Delta)$. Then $f^{-1}(\gamma)$ is a cobordism between $W_{z}$ 
and $W_{z^{\prime}}$ which amounts to adding one $n$-handle for each point $x \in \Delta$ such that $f(x) \subset \gamma$. More precisely, if $n>2$ and there is only one critical point $c \in f^{-1}(\gamma)$, then there exists $L \subset W_{z} \backslash B$ a framed Lagrangian sphere such that $W_{z^{\prime}}$ is the result of performing generalized Dehn surgery on $W_{z}$ along $L$. The framed sphere is the points in $W_{z}$ that converge to $c$ under parallel transport over $\gamma$.

Proof. Let $w \in \gamma$ and $c \in \Delta$ with $f(c)=w$. Let us take Morse coordinates around $c$ and an affine chart on $\mathbb{C P}^{1}$. Let us assume for simplicity that the curve $\gamma$ in the affine chart coincides with a segment of the real axis. For $r>0$ small enough, we want to construct a Poisson equivalence $\phi: W_{r} \rightarrow W_{-r}$.

To that end, consider the cobordism $Z=\tilde{f}^{-1}\left(x_{0}(-r, r)\right)$, which is a manifold with boundary because $\tilde{f}$ is transverse to $\gamma\left(\operatorname{Im} \sigma^{\prime}(0) \neq 0\right)$. The attaching of the handle in this elementary cobordism occurs in a neighborhood of $c$, or equivalently in a neighborhood of 0 in the Morse chart, which is where we work from now on.

We are going to arrange the current setting so that it becomes analogous to the one in Theorem 26.

The pullback of $f$ to the $t$-leaf of $\mathbb{C}^{n} \times \mathbb{R}$ is $h+\sigma(t)$. After reparametrization of the coordinate $t$, we may assume without loss of generality that $\sigma(t)=(a(t), t)$.

The tangent space of $Z$ at $0 \in \mathbb{C}^{n} \times \mathbb{R}$ is the hyperplane $t=0$. Therefore the projection $Z \rightarrow \mathbb{C}^{n}$ is a local diffeomorphism with image an open neighborhood $V$ of $0 \in \mathbb{C}^{n}$.

We define $\phi$ away from a neighborhood $V^{\prime} \subset V$ of $0 \in \mathbb{C}^{n}$ as follows:

$$
\phi:=\rho_{x_{0}(-r, r)}: W_{r} \rightarrow W_{-r} .
$$

We claim that it is possible to extend $\phi$ to an equivalence of 2-calibrated foliations repeating the proof of Theorem 26 with two minor modifications.

Let us define $\sigma_{r}(t):=(r, 0)+\sigma(t), r \neq 0, t \in[-\epsilon, \epsilon]$. Hence the images of $W_{r}$ and $W_{-r}$ on $V^{\prime}$ are exactly $h^{-1}\left(\sigma_{r}(t)\right)$ and $h^{-1}\left(\sigma_{-r}(t)\right)$, respectively. Recall that Morse coordinates on the $t$-plaque send $\omega_{\mathscr{F}}$ to a symplectic form $\Omega_{t}$, which makes the fibers of $h+\sigma(t)$ symplectic. Then it follows that the morphism $\rho_{x_{0}(-r, r)}: W_{r} \rightarrow W_{-} r$ at a point $p \in V^{\prime} \cap W_{r}$ in the $t$-leaf corresponds to parallel transport $\rho_{t, x_{t}}(r+a(t),-r+a(t))$ (with respect to $\Omega_{t}$ ).

The first modification we need to introduce is composing all curves used in the proof of Theorem 26 and defined in a neighborhood of $0 \in \mathbb{C}$ with the diffeomorphism $(x, y) \mapsto(x+a(y), y)$.

The second difference is that, from the very beginning, our parallel transport here is with respect to a family of symplectic forms $\Omega_{t}$, and with $\Omega_{0}$ of type $(1,1)$ at the origin. This situation is not quite new since in the proof of Theorem 26 we already needed to interpolate symplectic forms (although at a later stage).

Hence we conclude that for $r$ small enough the fiber $W_{-r}$ is equivalent to Lagrangian surgery (and hence by Theorem 26 generalized Dehn surgery) along 
a framed Lagrangian sphere $L$; the Lagrangian sphere is the points in $W_{r}$ which parallel transport over $x_{0}(r, 0)$ sends to the critical point $c$.

Remark 38. Theorem 37 is rather natural in view of the results for contact manifolds in [Presas 2002].

5.4. Further directions. In this paper we have shown that 2-calibrated foliations are a wide enough class of codimension-one foliations and, not surprisingly, techniques from symplectic geometry are well suited to their study. We would like to finish by discussing a couple of questions that we were not able to answer.

Theorem 4 shows that our embedded 3-dimensional taut foliations capture the leaf space of $\mathscr{F}$. What it would be interesting to know is whether they capture the full transverse geometry, that is, the holonomy groupoid.

A remarkable property of 3-dimensional taut foliations is that transverse loops are never nullhomotopic. The proof of this fact uses that the universal cover of the 3-manifold is $\mathbb{R}^{3}$, a property which does not extend to manifolds supporting a 2-calibrated foliation. We know no examples of 2-calibrated foliations on simply connected manifolds: in [Ibort and Martínez Torres 2003] it was shown that the normal connected sum could be used to construct 5-dimensional simply connected regular Poisson manifolds with codimension-one leaves, but those methods cannot be used to construct 2-calibrated foliations since the conditions in Theorem 10 are not fulfilled. It has been recently shown that Lawson's foliation on $S^{5}$ is the symplectic foliation of a Poisson structure [Mitsumatsu 2011]. However, this Poisson structure does not admit a 2-calibration because Lawson's foliation is not taut (the compact leaf would make any transverse loop nontrivial in homology).

We conjecture that any transverse loop in a 2-calibrated foliation is not nullhomotopic.

\section{Appendix: Legendrian surgery, open book decompositions and generalized Dehn surgery}

Let $(M, \xi)$ be an exact contact manifold and let $\alpha$ be a contact 1 -form defining $\xi=\operatorname{ker} \alpha$. Recall that an open book decomposition for $M$ is given by a pair $(K, \theta)$ such that

- $K$ is a codimension-2 submanifold with trivial normal bundle, referred to as the binding,

- $\theta: M \backslash K \rightarrow S^{1}$ is a fibration that in a trivialization $D^{2} \times K$ of a neighborhood of $K$ is the angular coordinate.

Let $F$ denote the closure of any fiber of $\theta$. The first return map associated to a suitable lift of $\partial / \partial \theta$ to $M \backslash K$ defines a diffeomorphism of $F$ supported away from a 
neighborhood of the boundary $\partial F=K$. Up to diffeomorphism $M$ can be recovered out of $F$ and the first return map.

The following discussion is mostly taken from [Giroux and Mohsen 2003]; alternatively, a less detailed account can be found in [Giroux 2002].

Definition 39. The contact structure $\xi$ is supported by an open book decomposition $(K, \theta)$ if for a choice of contact form $\alpha$ defining $\xi$ we have:

- $\alpha$ restricts to a contact form on $K$.

- $d \alpha$ restricts on each fiber of $\theta$ to an exact symplectic structure.

- The orientation of $K$ as the boundary of each symplectic leaf matches the natural orientation induced by the contact form.

The form $\alpha$ is said to be adapted to the open book decomposition $(K, \theta)$.

In what follows we are going to discuss contact structures and cosymplectic foliations on a given manifold. Since we have been using the notion of Reeb vector field for cosymplectic foliations, we refer to contact Reeb vector fields when discussing contact structures.

Given a contact form $\alpha$ adapted to $(K, \theta)$, it is possible to scale it away from $K$ to a contact 1-form $\alpha^{\prime}$ such that the flow along its contact Reeb vector field defines a compactly supported first return map $\varphi \in \operatorname{Symp}\left(\right.$ int $F, d \alpha^{\prime}$ ) [Giroux and Mohsen 2003].

The isotopy class of $(M, \xi)$ is totally determined by any open book decomposition supporting it [Giroux 2002; Giroux and Mohsen 2003]. More precisely, the relevant structure in the open book decomposition is the completion of the structure of exact symplectic manifold convex at infinity of the exact symplectic fiber (int $F, d \alpha$ ) (or (int $\left.F, d \alpha^{\prime}\right)$ ), together with the first return symplectomorphism supported inside int $F$.

The previous characterization becomes very important in light of the following theorem:

Theorem 40 [Giroux 2002; Giroux and Mohsen 2003]. For every exact contact manifold $(M, \xi)$ and any contact form defining $\alpha$, there exists an open book decomposition $(K, \theta)$ supporting $\xi$ such that $\alpha$ is adapted to it.

Let $\alpha$ be a contact form on $M$ adapted to the open book decomposition $(K, \theta)$ and let $L$ be a parametrized Legendrian sphere which is contained in a fiber of $\theta$, and hence it becomes Lagrangian for the symplectic structure $d \alpha$ on the fiber.

Observe that away from the binding $K$, the open book decomposition defines a 2-calibrated foliation $\left(M \backslash K, \mathscr{F}_{\theta}, d \alpha\right)$, with $\mathscr{F}_{\theta}=\operatorname{ker} d \theta$, which is a symplectic mapping torus associated to the symplectomorphism $\varphi$ supported in int $F$. Generalized Dehn surgery along $L$ produces a new symplectic mapping torus with return map $\varphi \circ \tau$, where $\tau$ is a generalized Dehn twist along $L$. Because the symplectic 
leaf is the same and the return map is still compactly supported, the symplectic mapping torus is in fact the open book decomposition of a unique contact manifold (up to isotopy). In [Giroux and Mohsen 2003] it has been announced that this contact manifold is $\left(M^{L}, \alpha^{L}\right)$, the result of performing Legendrian surgery along $L$ [Weinstein 1991]. (This is the same result involving plumbing along a Lagrangian disk announced in [Giroux 2002, p. 411].)

The ideas developed relating Lagrangian surgery and generalized Dehn surgery allow us to give a very natural proof of this result. The key step is the following theorem.

Theorem 41. Let $L \subset(M, \alpha)$ be a parametrized Legendrian sphere in a contact manifold and let $\left(M^{L}, \alpha^{L}\right)$ be the contact manifold obtained by Legendrian surgery along $L$. Suppose that $\alpha$ is adapted to the open book $(K, \theta)$ and that $L$ is contained in a fiber of $\theta$. Then given $V$ any small enough neighborhood of $L$ with empty intersection with the binding $K$, there exists an isotopy $\Psi_{s}: M \rightarrow M, s \in[0,1]$, starting at the identity with the following properties:

- $\Psi_{s}$ is supported inside $V$ and tangent to the identity at $L$.

- $\left(M \backslash K, \mathscr{F}_{\theta_{s}}, d \alpha\right)$, with $\mathscr{F}_{\theta_{s}}:=\Psi_{s *} \mathscr{F}_{\theta}$, is a 2-calibrated foliation and thus an open book decomposition $\left(K, \Psi_{s *} \theta\right)$ of $M$ to which the contact form $\alpha$ is adapted.

- Let $\left(M^{L} \backslash K, \mathscr{F}_{\theta_{1}^{L}}, d \alpha^{L}\right)$ be the result of performing generalized Dehn surgery on $\left(M \backslash K, \mathscr{F}_{\theta_{1}}, d \alpha\right)$ along the parametrized Lagrangian sphere L. Then $\left(M^{L} \backslash K, \mathscr{F}_{\theta_{1}^{L}}, d \alpha^{L}\right)$ is an open book decomposition $\left(K, \theta_{1}^{L}\right)$ for $M^{L}$ and the contact form $\alpha^{L} \in \Omega^{1}\left(M^{L}\right)$ is adapted to $\left(K, \theta_{1}^{L}\right)$.

Proof. We are going to recall Weinstein's definition of Legendrian surgery using symplectic cobordisms and a Liouville vector field transverse to the boundary. Actually, we will modify the original choices to make them compatible with our setup for Lagrangian surgery, or by Theorem 26 with the setup for generalized Dehn surgery.

Recall that a boundary component of a symplectic manifold $(Z, \Omega$ ) (of dimension bigger than 2) endowed with a Liouville vector field $Y$ is said to be convex if $Y$ is outward pointing and concave if $Y$ is inward pointing.

We consider $\left(M \times[-1,1], d\left(e^{v} \alpha\right)\right)$, which is a subset of the symplectization of $(M, \alpha)$. The tuple $\left(M \times[-1,1], d\left(e^{v} \alpha\right), \partial / \partial v, M \times\{0\}, L \times\{0\}\right)$ is an isotropic setup in the language of Weinstein; see the "Neighborhoods of isotropic submanifolds" section of [Weinstein 1991]. Note that $\{1\} \times M$ and $\{-1\} \times M$ are convex and concave boundary components, respectively (beware that the notion of Liouville vector field we use is opposite to Weinstein's, since we require the flow of the vector field to expand the symplectic form exponentially). 
The second isotropic setup is the one of the $(n+1)$-handle to be attached, which is the one described in the "Standard handle" section of [Weinstein 1991], up to the following change. Unlike Weinstein, we are going to glue the convex end of $\left(M \times[-1,1], d\left(e^{v} \alpha\right), \partial / \partial v, M \times\{0\}, L \times\{0\}\right)$ to the concave end of the symplectic $(n+1)$-handle; the reason is that in our definition of Lagrangian surgery, we glued the symplectic $(n+1)$-handle along the hypersurface $H_{2, r}$ where the symplectic vector field points inward. For this reason we also define a different Liouville vector field in the $(n+1)$-handle. We use the notation introduced in Section 4.1.

The symplectic form is the standard one $\Omega_{\mathbb{R}^{2 n+2}}$. We consider the function

$$
q=\sum_{i=1}^{n+1} x_{i}^{2}-2 y_{i}^{2},
$$

whose negative gradient with respect to the Euclidean metric,

$$
E=-2 x^{1} \frac{\partial}{\partial x^{1}}+4 y^{1} \frac{\partial}{\partial y^{1}}-\cdots-2 x^{n+1} \frac{\partial}{\partial x^{n+1}}+4 y^{n+1} \frac{\partial}{\partial y^{n+1}},
$$

is a Liouville vector field.

For each $r>0$ we consider the fiber $q_{r}$, which contains the Lagrangian sphere $\Sigma_{r}$ described in Lemma 18 using $Y_{2}$ the Hamiltonian vector field of $-\operatorname{Re} h$ with respect to $\Omega_{\mathbb{R}^{2 n+2}}$. Notice that $d q\left(Y_{2}\right)<0$ and therefore $Y_{2}$ is transverse to the level hypersurfaces $q_{r}$. Since $Y_{2}$ and $E$ coincide at $\Sigma_{r}$, it follows that the sphere $\Sigma_{r}$ is also Legendrian with respect to the contact form $\alpha_{E}:=i_{E} \Omega_{\mathbb{R}^{2 n+2}}$ on $q_{r}$. Moreover, at points of $\Sigma_{r} \subset q_{r}$ the contact distribution and the cosymplectic distribution coincide.

Let $V_{r}(\epsilon)$ be a tubular neighborhood of radius $\epsilon>0$ of $\Sigma_{r}$ inside $q_{r}$ with respect to the Euclidean metric. We claim that for any $\epsilon^{\prime}>0, \epsilon>\epsilon^{\prime}$, we have $f_{r} \in C^{\infty}\left(V_{r}(\epsilon) \backslash \Sigma_{r}, \mathbb{R}^{+}\right)$a cut-off function with compact support and with the following two properties:

- $\Phi_{1}^{f_{r} Y_{2}}\left(V_{r}\left(\epsilon^{\prime}\right) \backslash \Sigma_{r}\right) \subset q_{-2 r}$ (note that $q_{-2 r}$ contains the Lagrangian sphere $\Sigma_{-r}$ ).

- $\Phi_{1}^{f_{r} Y_{2}}\left(V_{r}(\epsilon)\right)$ is transverse to $E$.

Assuming the claim, we define the hypersurface

$$
H_{r}^{L}:=\Phi_{1}^{f_{r} Y_{2}}\left(V_{r}(\epsilon) \backslash \Sigma_{r}\right) \cup \Sigma_{-r} .
$$

By assumption the Liouville vector field $E$ is transverse to $H_{r}^{L}$, and thus the hypersurface inherits an exact contact structure $\alpha_{E}$ by restricting $i_{E} \Omega_{\mathbb{R}^{2 n+2}}$.

The second isotropic setup is the following: the symplectic $(n+1)$-handle is the compact region bounded by $H_{r}^{L}$ and $V_{r}(\epsilon)$ endowed with the standard symplectic form, the Liouville vector field is $E$, the hypersurface is $V_{r}(\epsilon)$, which is concave, and the parametrized Legendrian sphere is $\Sigma_{r}$. 
The symplectic morphism $\psi$ that gives rise to the symplectic elementary cobordism ([Weinstein 1991, Proposition 4.2], whose replacement for Lagrangian surgery is Lemma 16), sends $\left(V_{r}(\epsilon), \Sigma_{r}, \alpha_{E}\right)$ to $(\nu(L), L, \alpha)$, and therefore we can consider $\left(V_{r}(\epsilon), \Sigma_{r}, \alpha_{E}\right)$ as a subset of $(M, \alpha)$. Then $M^{L}:=H_{r}^{L} \cup\left(M \backslash V_{r}(\epsilon)\right)$ carries and obvious contact form $\alpha^{L}$ which extends $\left(M \backslash V_{r}(\epsilon), \alpha\right)$.

The data for Legendrian surgery has been chosen to be compatible with Lagrangian surgery: both $H_{r}^{L}$ and $V_{r}(\epsilon)$ are transverse to $Y_{2}$ and therefore they inherit 2-calibrated foliations $\left(H_{r}^{L}, \mathscr{F}_{r}^{L}, \omega_{r}^{L}\right)$ and $\left(V_{r}(\epsilon), \mathscr{F}_{r}, d \alpha\right)$. Theorem 26 easily implies that $\left(H_{r}^{L}, \mathscr{F}_{r}^{L}, \omega_{r}^{L}\right)$ is the result of generalized Dehn surgery along $\Sigma_{r} \subset\left(V_{r}(\epsilon), \mathscr{F}_{r}, d \alpha\right)$.

On $V_{r}(\epsilon)$ we have two structures of 2-calibrated foliation, $\left(\mathscr{F}_{r}, d \alpha\right)$ and $(\mathscr{F}, d \alpha)$. The reason is that $\psi$ preserves contact forms and hence contact Reeb vector fields, but it does not preserve the 1-forms defining the cosymplectic foliations (or their associated Reeb vector fields). However, at $\Sigma_{r}$ the Liouville and Hamiltonian vector fields coincide, and this implies that at points in $L$ the contact distribution is tangent to $\mathscr{F}_{r}$. In particular the contact Reeb vector field for $\alpha$ is transverse to $\mathscr{F}_{r}$ near $L$. It is also transverse to $\mathscr{F}$ because $\alpha$ is adapted to the open book. Therefore we can use the trajectories of the contact Reeb vector field to construct an isotopy $\Psi_{s}$ tangent to the identity at $L$ and supported inside $V$ in a small neighborhood of $\Sigma_{r}$ contained in $V_{r}(\epsilon)$.

The claim about the existence of the function $f_{r}$ is easily proved when $n=1$ by inspecting the trajectories of $E$ and $Y_{2}$. The general case can be reduced to the previous one: each point $\left(x_{1}, y_{1}, \ldots, x_{n+1}, y_{n+1}\right)$ in $\mathbb{C}^{n+1}$ and away from the union of stable and unstable manifolds (these are the same for both Morse functions $\operatorname{Re} h$ and $q$ ) determines $\left[x_{1}: \cdots: x_{n+1}\right],\left[y_{1}: \cdots: y_{n+1}\right]$, a point in $\mathbb{R} \mathbb{P}^{n} \times \mathbb{R} \mathbb{P}^{n}$, which gives rise to a line in $\mathbb{R}^{n+1}$ and one in $i \mathbb{R}^{n+1}$. These lines span a plane in $\mathbb{C}^{n+1}=\mathbb{R} \oplus i \mathbb{R}^{n+1}$. Each plane in the family is preserved by the flow of $E$ and $Y_{2}$; moreover, the flows restrict on the planes to the flows of the 1-dimensional case. From this observation the claim follows easily.

Theorem 41 provides an isotopy $\Psi_{s}$ supported away from $K$ so that $\alpha$ is adapted to the 1-parameter family of open book decompositions $\left(K, \Psi_{s} \theta\right)$. Therefore we can identify the symplectic fiber $F$ and symplectic monodromy $\varphi \in$ Symp(int $F, d \alpha)$ of $(K, \theta)$ with those of $\left(K, \Psi_{1} \theta\right)$ (again following the contact Reeb flow). Hence the third point in Theorem 41 asserts that $\left(M^{L}, \alpha^{L}\right)$ is adapted to an open book decomposition with the same symplectic leaf $(F, d \alpha)$ and monodromy $\varphi \circ \tau \in \operatorname{Symp}(\operatorname{int} F, d \alpha$ ), which is exactly what we wanted to prove.

Remark 42. If we attach the convex end of the symplectic handle to the concave end of the symplectization, we get the contact manifold $\left(M^{L^{-}}, \alpha^{L^{-}}\right) \cdot \alpha^{L^{-}}$is adapted to an open book decomposition whose monodromy is $\varphi \circ \tau^{-1}$. 
Proposition 6.1 of [Durfee and Kauffman 1975] implies that in dimensions 5 and 13 the manifolds $M^{L}$ and $M^{L^{-}}$are diffeomorphic. In [van Koert and Niederkrüger 2005, Section 3], it is shown that there are instances (coming from Brieskorn manifolds) in which $\left(M^{L}, \alpha^{L}\right)$ and $\left(M^{L^{-}}, \alpha^{L^{-}}\right)$are not contactomorphic, and hence the authors can deduce that $\tau^{2}$ is not isotopic to the identity in $\operatorname{Symp}{ }^{\text {comp }}\left(T^{*} S^{6}, d \alpha_{\text {can }}\right)$, a result already proved by Seidel for $n=2$ [1999]; similar results are also drawn for powers of the Dehn twists known to be isotopic to the identity in $\operatorname{Diff}^{\text {comp }}(T(\lambda))$, for all $n$ even.

Remark 43. For any contact form $\alpha$ on $M$ representing the given contact structure $\xi$ and $L$ a Legendrian submanifold, Giroux and Mohsen [2003] announce the existence of relative open book decompositions, meaning that $\alpha$ is adapted to the open book decomposition and $L$ is contained in a fiber.

The interested reader familiar with approximately holomorphic geometry [Donaldson 1996] and its version for contact manifolds [Ibort et al. 2000; Presas 2002] can write a proof along the following lines: the open book decomposition is the result of pulling back the canonical open book decomposition of $\mathbb{C}$ by an approximately holomorphic function. To make sure the binding does not contain $L$, we use reference sections supported near $L$ which achieve the value 1 when restricted to $L$; they come from an explicit formula once we identify a tubular neighborhood of $L$ with a tubular neighborhood of the zero section of the first jet bundle with its canonical contact structure $\left(\mathscr{F}^{1} L, \alpha_{\text {can }}\right)$. It is necessary to further add perturbations whose restrictions to $L$ attain real values: they are such that its restrictions to $T^{*} L \times\{0\} \subset \mathscr{F}^{1} L$ are small real multiples of reference sections equivariant with respect to the involution on $\left(\mathscr{F}^{1}, \alpha_{\mathrm{can}}\right)$ that reverses the sign of the fiber and conjugation on $\mathbb{C}$ (this construction is analogous to the content of the remark after Lemma 3 in [Auroux et al. 2001]).

Therefore we conclude that Lagrangian surgery includes Legendrian surgery, for we can bypass the latter by choosing appropriate compatible open book decompositions and then performing Lagrangian surgery. According to Theorem 26 we can even claim that generalized Dehn surgery contains Legendrian surgery, and forget about the cobordisms.

Actually, generalized Dehn surgeries for different open book decompositions supporting the contact structure give the same contact manifold because there is a contact surgery behind. Now consider $(L, \chi)$ where $L$ is a Legendrian submanifold of $(M, \alpha)$ and $\chi \in \operatorname{Symp}^{\text {comp }}\left(T^{*} L, d \alpha_{\text {can }}\right)$. Let us take any open book decomposition relative to $L$ and such that $\alpha$ is adapted to it, and consider the new manifold $M^{L}$ associated to the open book decomposition with symplectic monodromy $\varphi \circ \chi$. It is clear that the diffeomorphism type of the manifold does not depend on the open book decomposition, but it is not clear whether in general the contact structure depends on the choice of open book decomposition. In either case, it would be an 
interesting situation because it would give either a new contact surgery - possibly a Legendrian surgery based on a block different from a symplectic handle — or different contact structures.

\section{Acknowledgments}

The author is very grateful to the referee for corrections and numerous suggestions.

\section{References}

[Alcalde-Cuesta and Hector 1993] F. Alcalde-Cuesta and G. Hector, Intégration symplectique des variétés de Poisson régulières sans cycle évanouissant, thesis, Université Claude Bernard-Lyon I Institut de Mathématique et Informatique, 1993.

[Auroux 1997] D. Auroux, "Asymptotically holomorphic families of symplectic submanifolds", Geom. Funct. Anal. 7:6 (1997), 971-995. MR 99b:57069 Zbl 0912.53020

[Auroux et al. 2001] D. Auroux, D. Gayet, and J.-P. Mohsen, "Symplectic hypersurfaces in the complement of an isotropic submanifold", Math. Ann. 321:4 (2001), 739-754. MR 2002j:53113 Zbl 1005.53049

[Bertelson 2001] M. Bertelson, "Foliations associated to regular Poisson structures", Commun. Contemp. Math. 3:3 (2001), 441-456. MR 2002i:53110 Zbl 1002.53056

[Bertelson 2002] M. Bertelson, "A $h$-principle for open relations invariant under foliated isotopies", J. Symplectic Geom. 1:2 (2002), 369-425. MR 2004k:53133 Zbl 1036.53017

[Donaldson 1996] S. K. Donaldson, "Symplectic submanifolds and almost-complex geometry", J. Differential Geom. 44:4 (1996), 666-705. MR 98h:53045 Zbl 0883.53032

[Durfee and Kauffman 1975] A. Durfee and L. Kauffman, "Periodicity of branched cyclic covers", Math. Ann. 218:2 (1975), 157-174. MR 52 \#6731 Zbl 0296.55001

[El Kacimi-Alaoui 1983] A. El Kacimi-Alaoui, "Sur la cohomologie feuilletée", Compositio Math. 49:2 (1983), 195-215. MR 85a:57016 Zbl 0516.57017

[Fernandes and Frejlich 2012] R. L. Fernandes and P. Frejlich, "An $h$-principle for symplectic foliations", Int. Math. Res. Not. 2012:7 (2012), 1505-1518. MR 2913182 Zbl 1245.53033

[Giroux 2002] E. Giroux, "Géométrie de contact: de la dimension trois vers les dimensions supérieures", pp. 405-414 in Proceedings of the International Congress of Mathematicians (Beijing, 2002), vol. 2, edited by T. Li, Higher Education Press, Beijing, 2002. MR 2004c:53144 Zbl 1015.53049

[Giroux and Mohsen 2003] E. Giroux and J.-P. Mohsen, "Structures de contacte et fibrations symplectiques au-dessus du cercle", lecture notes, 2003.

[Gompf 1995] R. E. Gompf, "A new construction of symplectic manifolds", Ann. of Math. (2) 142:3 (1995), 527-595. MR 96j:57025 Zbl 0849.53027

[Gompf and Stipsicz 1999] R. E. Gompf and A. I. Stipsicz, 4-manifolds and Kirby calculus, Graduate Studies in Mathematics 20, American Mathematical Society, Providence, RI, 1999. MR 2000h:57038 Zbl 0933.57020

[Gotay 1982] M. J. Gotay, "On coisotropic imbeddings of presymplectic manifolds", Proc. Amer. Math. Soc. 84:1 (1982), 111-114. MR 83j:53028

[Greene and Shiohama 1979] R. E. Greene and K. Shiohama, "Diffeomorphisms and volumepreserving embeddings of noncompact manifolds", Trans. Amer. Math. Soc. 255 (1979), 403-414. MR 80k:58031 Zbl 0418.58002 
[Guillemin et al. 2011] V. Guillemin, E. Miranda, and A. R. Pires, "Codimension one symplectic foliations and regular Poisson structures", Bull. Braz. Math. Soc. (N.S.) 42:4 (2011), 607-623. MR 2861781 Zbl 1244.53093

[Harvey and Lawson 1982] R. Harvey and H. B. Lawson, Jr., "Calibrated foliations (foliations and mass-minimizing currents)”, Amer. J. Math. 104:3 (1982), 607-633. MR 84h:53095 Zbl 0508.57021

[Hofer and Zehnder 1994] H. Hofer and E. Zehnder, Symplectic invariants and Hamiltonian dynamics, Birkhäuser, Basel, 1994. MR 96g:58001 Zbl 0805.58003

[Ibort and Martínez Torres 2003] A. Ibort and D. Martínez Torres, "A new construction of Poisson manifolds”, J. Symplectic Geom. 2:1 (2003), 83-107. MR 2007a:53151 Zbl 1063.53082

[Ibort and Martínez Torres 2004a] A. Ibort and D. Martínez Torres, "Approximately holomorphic geometry and estimated transversality on 2-calibrated manifolds", C. R. Math. Acad. Sci. Paris 338:9 (2004), 709-712. MR 2005f:53154 Zbl 1057.53043

[Ibort and Martínez Torres 2004b] A. Ibort and D. Martínez Torres, "Lefschetz pencil structures for 2-calibrated manifolds", C. R. Math. Acad. Sci. Paris 339:3 (2004), 215-218. MR 2005d:53138 Zbl 1054.57029

[Ibort et al. 2000] A. Ibort, D. Martínez-Torres, and F. Presas, "On the construction of contact submanifolds with prescribed topology", J. Differential Geom. 56:2 (2000), 235-283. MR 2003f:53158 Zbl 1034.53088

[van Koert and Niederkrüger 2005] O. van Koert and K. Niederkrüger, "Open book decompositions for contact structures on Brieskorn manifolds", Proc. Amer. Math. Soc. 133:12 (2005), 3679-3686. MR 2006f:53135 Zbl 1083.53077

[Lickorish 1965] W. B. R. Lickorish, "A foliation for 3-manifolds", Ann. of Math. (2) 82 (1965), 414-420. MR 32 \#6488 Zbl 0142.41104

[McDuff and Salamon 1998] D. McDuff and D. Salamon, Introduction to symplectic topology, 2nd ed., Oxford University Press, New York, 1998. MR 2000g:53098 Zbl 1066.53137

[Melvin 1984] P. Melvin, "2-sphere bundles over compact surfaces”, Proc. Amer. Math. Soc. 92:4 (1984), 567-572. MR 85j:57039 Zbl 0524.55016

[Mitsumatsu 2011] Y. Mitsumatsu, "Leafwise symplectic structures on Lawson's foliation”, preprint, 2011. arXiv 1101.2319

[Ohsawa and Sibony 2000] T. Ohsawa and N. Sibony, "Kähler identity on Levi flat manifolds and application to the embedding”, Nagoya Math. J. 158 (2000), 87-93. MR 2001d:32055 Zbl 0976.32021

[Palis and de Melo 1982] J. Palis, Jr. and W. de Melo, Geometric theory of dynamical systems: An introduction, Springer, New York, 1982. MR 84a:58004 Zbl 0491.58001

[Presas 2002] F. Presas, "Lefschetz type pencils on contact manifolds", Asian J. Math. 6:2 (2002), 277-301. MR 2003g:57043 Zbl 1101.53055

[Rummler 1979] H. Rummler, "Quelques notions simples en géométrie riemannienne et leurs applications aux feuilletages compacts", Comment. Math. Helv. 54:2 (1979), 224-239. MR 80m:57021 Zbl 0409.57026

[Seidel 1999] P. Seidel, "Lagrangian two-spheres can be symplectically knotted", J. Differential Geom. 52:1 (1999), 145-171. MR 2001g:53139 Zbl 1032.53068

[Seidel 2000] P. Seidel, “Graded Lagrangian submanifolds”, Bull. Soc. Math. France 128:1 (2000), 103-149. MR 2001c:53114 Zbl 0992.53059

[Seidel 2003] P. Seidel, "A long exact sequence for symplectic Floer cohomology", Topology 42:5 (2003), 1003-1063. MR 2004d:53105 Zbl 1032.57035 
[Sullivan 1976] D. Sullivan, "Cycles for the dynamical study of foliated manifolds and complex manifolds", Invent. Math. 36 (1976), 225-255. MR 55 \#6440 Zbl 0335.57015

[Thurston 1976] W. P. Thurston, "Existence of codimension-one foliations", Ann. of Math. (2) 104:2 (1976), 249-268. MR 54 \#13934 Zbl 0347.57014

[Weinstein 1991] A. Weinstein, "Contact surgery and symplectic handlebodies", Hokkaido Math. J. 20:2 (1991), 241-251. MR 92g:53028 Zbl 0737.57012

Received August 19, 2011. Revised June 10, 2012.

DAVID MARTÍNEZ TORRES

Centro de Análise Matemática, Geometria e Sistemas Dinâmicos

DEPARTAMENTO DE MATEMÁTICA

INSTITUTO SUPERIOR TÉCNICO

AV. Rovisco PAIS

1049-001 LISBON

PORTUGAL

martinez@math.ist.utl.pt 


\title{
PACIFIC JOURNAL OF MATHEMATICS
}

\author{
msp.org/pjm
}

Founded in 1951 by E. F. Beckenbach (1906-1982) and F. Wolf (1904-1989)

\section{EDITORS}

V. S. Varadarajan (Managing Editor)

Department of Mathematics

University of California

Los Angeles, CA 90095-1555

pacific@math.ucla.edu

Paul Balmer

Department of Mathematics

University of California

Los Angeles, CA 90095-1555

balmer@math.ucla.edu

Daryl Cooper

Department of Mathematics

University of California

Santa Barbara, CA 93106-3080 cooper@math.ucsb.edu

Jiang-Hua $\mathrm{Lu}$

Department of Mathematics

The University of Hong Kong

Pokfulam Rd., Hong Kong jhlu@maths.hku.hk
Don Blasius

Department of Mathematics University of California

Los Angeles, CA 90095-1555

blasius@math.ucla.edu

Robert Finn

Department of Mathematics Stanford University

Stanford, CA 94305-2125

finn@math.stanford.edu

Sorin Popa

Department of Mathematics

University of California

Los Angeles, CA 90095-1555

popa@math.ucla.edu

Paul Yang

Department of Mathematics

Princeton University

Princeton NJ 08544-1000

yang@math.princeton.edu

\section{PRODUCTION}

Silvio Levy, Scientific Editor, production@msp.org

\section{SUPPORTING INSTITUTIONS}

ACADEMIA SINICA, TAIPEI

CALIFORNIA INST. OF TECHNOLOGY

INST. DE MATEMÁTICA PURA E APLICADA

KEIO UNIVERSITY

MATH. SCIENCES RESEARCH INSTITUTE

NEW MEXICO STATE UNIV.

OREGON STATE UNIV.

\author{
STANFORD UNIVERSITY \\ UNIV. OF BRITISH COLUMBIA \\ UNIV. OF CALIFORNIA, BERKELEY \\ UNIV. OF CALIFORNIA, DAVIS \\ UNIV. OF CALIFORNIA, LOS ANGELES \\ UNIV. OF CALIFORNIA, RIVERSIDE \\ UNIV. OF CALIFORNIA, SAN DIEGO \\ UNIV. OF CALIF., SANTA BARBARA
}

\author{
Vyjayanthi Chari \\ Department of Mathematics \\ University of California \\ Riverside, CA 92521-0135 \\ chari@math.ucr.edu \\ Kefeng Liu \\ Department of Mathematics \\ University of California \\ Los Angeles, CA 90095-1555 \\ liu@math.ucla.edu \\ Jie Qing \\ Department of Mathematics \\ University of California \\ Santa Cruz, CA 95064 \\ qing@cats.ucsc.edu
}

These supporting institutions contribute to the cost of publication of this Journal, but they are not owners or publishers and have no responsibility for its contents or policies.

See inside back cover or msp.org/pjm for submission instructions.

The subscription price for 2013 is US \$400/year for the electronic version, and \$485/year for print and electronic.

Subscriptions, requests for back issues and changes of subscribers address should be sent to Pacific Journal of Mathematics, P.O. Box 4163, Berkeley, CA 94704-0163, U.S.A. The Pacific Journal of Mathematics is indexed by Mathematical Reviews, Zentralblatt MATH, PASCAL CNRS Index, Referativnyi Zhurnal, Current Mathematical Publications and the Science Citation Index.

The Pacific Journal of Mathematics (ISSN 0030-8730) at the University of California, c/o Department of Mathematics, 798 Evans Hall \#3840, Berkeley, CA 94720-3840, is published monthly except July and August. Periodical rate postage paid at Berkeley, CA 94704, and additional mailing offices. POSTMASTER: send address changes to Pacific Journal of Mathematics, P.O. Box 4163, Berkeley, CA 94704-0163.

PJM peer review and production are managed by EditFLOW ${ }^{\circledR}$ from Mathematical Sciences Publishers.

PUBLISHED BY

mathematical sciences publishers

nonprofit scientific publishing

http://msp.org/

(C) 2013 Mathematical Sciences Publishers 


\section{PACIFIC JOURNAL OF MATHEMATICS}

Volume $261 \quad$ No. $1 \quad$ January 2013

Hierarchies and compatibility on Courant algebroids

Paulo Antunes, Camille Laurent-GengouX and

JoANA M. NunES DA COSTA

A new characterization of complete linear Weingarten hypersurfaces in real 33 space forms

Cícero P. Aquino, Henrique F. DE Lima and

MARCO A. L. VELÁSQUEZ

Calogero-Moser versus Kazhdan-Lusztig cells

CÉDRIC BONNAFÉ and RAPHAËL ROUQUIER

Coarse median spaces and groups

BRIAN H. BOWDITCH

Geometrization of continuous characters of $\mathbb{Z}_{p}^{\times}$

CLIFTON CUNNINGHAM and MASOUd KAMGARPOUR

A note on Lagrangian cobordisms between Legendrian submanifolds of $\mathbb{R}^{2 n+1}$

\section{ROMAN GOLOVKO}

On slope genera of knotted tori in 4-space

Yi LIU, Yi Ni, HoNGBIN SUN and SHICHENG WANG

Formal groups of elliptic curves with potential good supersingular reduction

$$
\text { ÁlVARO LOZANO-ROBLEDO }
$$

Codimension-one foliations calibrated by nondegenerate closed 2-forms

DAVID MARTínez TORRES

The trace of Frobenius of elliptic curves and the $p$-adic gamma function

DERMOT MCCARTHY

$(D N)-(\Omega)$-type conditions for Fréchet operator spaces 